\title{
Double Cuvet-ISES: In Situ Estimation of Enantioselectivity and Relative Rate for Catalyst Screening
}

\author{
Sangeeta Dey, Kannan R. Karukurichi, Weijun Shen and David B. Berkowitz* \\ Department of Chemistry, University of Nebraska, Lincoln, NE 68588-0304 \\ dbb@unlserve.unl.edu
}

\section{SUPPORTING INFORMATION}

\section{General Experimental}

All reactions were conducted under argon atmosphere using flame or oven-dried glassware, unless otherwise indicated. Methylene chloride was distilled from $\mathrm{CaH}_{2}$. Toluene, THF and $\mathrm{Et}_{2} \mathrm{O}$ were distilled from sodium benzophenone ketyl. Methanol was distilled from $\mathrm{Mg}$, and ethanol from Nadiethyl phthalate. Alcohol dehydrogenase from equine liver (HLADH, EC 1.1.1.1), alcohol dehydrogenase from Thermoanaerobium brockii (TBADH, EC 1.1.1.2), $\beta-\mathrm{NAD}^{+}$(sodium salt) and $\beta$ $\mathrm{NADP}^{+}$(sodium salt) were purchased from Sigma. 3,5-Di-tert-butylsalicylaldehyde (a) was purchased from Alfa-Aesar, 3-tert-butylsalicylaldehyde (b), 2-hydroxy-1-naphthaldehyde (c) and cobalt(II)acetate tetrahydrate from Aldrich, 1-hydroxy-2-naphthaldehyde (d) from TCI America, 3,5diiodosalicylaldehyde (e), 4-benzyloxysalicylaldehyde (f) from Lancaster and 8-hydroxy-1,1,7,7tetramethyljulolidinyl-9-carboxaldehyde (g) from Acros. Flash chromatography was performed using Merck silica gel 60 (230-400 mesh). ${ }^{1} \mathrm{H}$ NMR spectra were recorded on Bruker-DRX-Avance-400 $\mathrm{MHz}, 500 \mathrm{MHz}$ and $600 \mathrm{MHz}$ instruments with chemical shifts reported relative to residual $\mathrm{CHCl}_{3}$ (7.25 ppm) and $\mathrm{CH}_{2} \mathrm{Cl}_{2}(5.2 \mathrm{ppm})$. Proton-decoupled ${ }^{13} \mathrm{C}$ NMR spectra were acquired on Bruker-DRXAvance-400 MHz, $500 \mathrm{MHz}$ and $600 \mathrm{MHz}$ instruments with chemical shifts reported relative to $\mathrm{CDCl}_{3}$ (77.0 ppm). Optical rotation @ $589 \mathrm{~nm}$ was measured at $19{ }^{\circ} \mathrm{C}$ in an Autopol polarimeter. IR spectra were obtained using a Nicolet Avatar 360 FTIR spectrometer. Mass spectra were acquired at the Nebraska Center for Mass Spectrometry (University of Nebraska-Lincoln). Enzyme assays and ISES were done in a Shimadzu UV-2101PC spectrophotometer equipped with a CPS-260 six cell positioner and thermoelectric temperature control (set at $25{ }^{\circ} \mathrm{C}$ for all experiments reported), or a Shimadzu 2401 spectrophotometer, equipped with a 12-cell changer and water-jacketed cell holder for temperature control. Enantiomeric excesses were determined using a Chiralcel OD $(0.46 \mathrm{~mm}$ x $25 \mathrm{~cm})$ chiral column. 


\section{Synthetic Chemistry}

General Procedure A: Synthesis of Chiral 'Salen' Ligands (illustrated for 2a).

An oven-dried RB flask was charged with (1S)-phenyl-1,2-ethylenediamine (250 mg, $1.83 \mathrm{mmol})$ and 3,5-di-tert-butylsalicylaldehyde $(861 \mathrm{mg}, 3.67 \mathrm{mmol})$ and freshly distilled ethanol $(2 \mathrm{~mL})$. The reaction mixture was stirred at $45^{\circ} \mathrm{C}$ for $1 \mathrm{~d}$ whereupon, in most cases, the salen product crystallized out. The reaction temperature and time varied, as is indicated in the individual procedures. In the case of $\mathbf{2 a}$, the product could be obtained in pure form $(823 \mathrm{mg}, 79 \%)$ by simple trituration with cold ethanol. In other cases, if further purification was required, this was achieved by recrystallization or column chromatography.

\section{$N, N^{\prime}$-Bis $(3 ', 5$ '-di-tert-butylsalicylidene[1R-(1 $\left.\alpha, 2 \alpha, 5 \alpha)\right]-2$-amino-7,7-dimethyl-2-}

bicyclo[3.3.1]heptane-ethanamine (1a). Diamine 1 was prepared from commercially available $(1 S)$ (-)- $\beta$-pinene as described. ${ }^{1}$ Following General Procedure A, from diamine 1 (43.3 mg, $0.32 \mathrm{mmol}$ ) and 3,5-di-tert-butylsalicylaldehyde $(150 \mathrm{mg}, 0.64 \mathrm{mmol})$, after heating at $40-50{ }^{\circ} \mathrm{C}$ for $5 \mathrm{~h}$, filtration and trituration (cold EtOH), was obtained clean $\left.1 \mathrm{a}(140 \mathrm{mg}, 92 \%):[\alpha]^{19}{ }_{\mathrm{D}}+14.2(c) 3.54, \mathrm{CH}_{2} \mathrm{Cl}_{2}\right) ;{ }^{1} \mathrm{H}$ NMR (400 MHz, $\left.\mathrm{CDCl}_{3}\right) \delta 1.23(\mathrm{~d}, J=10 \mathrm{~Hz}, 1 \mathrm{H}), 1.23(\mathrm{~s}, 3 \mathrm{H}), 1.26(\mathrm{~s}, 9 \mathrm{H}), 1.26(\mathrm{~s}, 9 \mathrm{H}), 1.36(\mathrm{~s}, 3 \mathrm{H})$, $1.43(\mathrm{~s}, 9 \mathrm{H}), 1.43(\mathrm{~s}, 9 \mathrm{H}), 1.92-1.97(\mathrm{~m}, 3 \mathrm{H}), 2.03$ (app t, $J=11,10 \mathrm{~Hz}, 1 \mathrm{H}), 2.17-2.26(\mathrm{~m}, 2 \mathrm{H}), 2.28-$ $2.32(\mathrm{~m}, 1 \mathrm{H}), 2.42(\mathrm{t}, J=5.5 \mathrm{~Hz}, 1 \mathrm{H}), 3.70(\mathrm{~d}, J=8 \mathrm{~Hz}, 1 \mathrm{H}), 3.91(\mathrm{~d}, J=8 \mathrm{~Hz}, 1 \mathrm{H}), 6.98(\mathrm{~d}, J=2 \mathrm{~Hz}$, $1 \mathrm{H}), 7.04(\mathrm{~d}, J=2 \mathrm{~Hz}, 1 \mathrm{H}), 7.32(\mathrm{~d}, J=2 \mathrm{~Hz}, 1 \mathrm{H}), 7.33(\mathrm{~d}, J=2 \mathrm{~Hz}, 1 \mathrm{H}), 8.25(\mathrm{~s}, 1 \mathrm{H}), 8.264(\mathrm{~s}, 1 \mathrm{H})$,

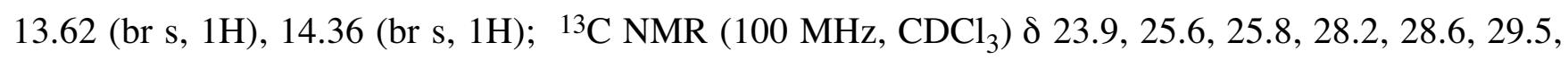
31.47, 31.49, 34.1, 35.0, 35.1, 38.5, 40.0, 48.6, 67.2, 68.8, 117.8, 117.8, 126.0, 126.4, 126.6, 126.9, 136.5, 136.5, 139.6, 139.9, 158.1, 158.6, 162.69, 167.71; HRMS (FAB, 3-NOBA) calcd for $\mathrm{C}_{40} \mathrm{H}_{61} \mathrm{~N}_{2} \mathrm{O}_{2}(\mathrm{M}+\mathrm{H})^{+} 601.4733$, obsd. 601.4710 .

\section{$N, N^{\prime}$-Bis(3'-tert-butylsalicylidene)-[1R-(1 $\left.\left.\alpha, 2 \alpha, 5 \alpha\right)\right]$-2-amino-7,7-dimethyl-2-bicyclo[3.3.1]heptane-}

ethanamine (1b). Following General Procedure A, from diamine 1 (101 mg, $0.60 \mathrm{mmol}$ ) and 3-tertbutyl-2-hydroxybenzaldehyde $(214 \mathrm{mg}, 1.20 \mathrm{mmol})$, and chromatographic purification $\left(5 \% \mathrm{Et}_{3} \mathrm{~N}\right.$ hexanes), was obtained compound $1 \mathbf{b}(180 \mathrm{mg}, 61 \%)$ : $[\alpha]{ }^{19} \mathrm{D}+2.66\left(c 4.65, \mathrm{CH}_{2} \mathrm{Cl}_{2}\right) ;{ }^{1} \mathrm{H}$ NMR $(400$ $\left.\mathrm{MHz}_{\mathrm{CDCl}}\right) \delta 1.05(\mathrm{~d}, J=10.8 \mathrm{~Hz}, 1 \mathrm{H}), 1.23(\mathrm{~s}, 21 \mathrm{H}), 1.35(\mathrm{~s}, 3 \mathrm{H}) 1.89-2.06(\mathrm{~m}, 3 \mathrm{H}), 2.13-2.31(\mathrm{~m}$, $3 \mathrm{H}), 2.41(\operatorname{app~t}, J=5.6,5 \mathrm{~Hz}, 1 \mathrm{H}), 3.76(\mathrm{~d}, J=12 \mathrm{~Hz}, 1 \mathrm{H}), 3.86(\mathrm{~d}, J=12 \mathrm{~Hz}, 1 \mathrm{H}), 6.84(\mathrm{t}, J=8 \mathrm{~Hz}$, $2 \mathrm{H}), 7.10(\mathrm{dd}, J=9.0,2 \mathrm{~Hz}, 2 \mathrm{H}), 7.29(\mathrm{dt}, J=9,2 \mathrm{~Hz}, 2 \mathrm{H}), 8.13(\mathrm{~s}, 1 \mathrm{H}), 8.17(\mathrm{~s}, 1 \mathrm{H}), 13.12(\mathrm{~s}, 1 \mathrm{H})$, $13.63(\mathrm{~s}, 1 \mathrm{H}) ;{ }^{13} \mathrm{C} \mathrm{NMR}\left(100 \mathrm{MHz}, \mathrm{CD}_{2} \mathrm{Cl}_{2}\right) \delta$ 23.8, 25.7, 28.2, 28.5, 31.39, 31.41, 33.9, 38.4, 40.0, 46.3, 48.3, 67.6, 68.9, 116.4, 166.5, 117.8, 117.9, 127.9, 129.4, 129.7, 140.9, 141.2, 158.7, 159.2, 161.8, 167.1; HRMS (FAB, 3-NOBA) calcd for $\mathrm{C}_{32} \mathrm{H}_{45} \mathrm{O}_{2} \mathrm{~N}_{2}(\mathrm{M}+\mathrm{H})^{+} 489.3481$, obsd 489.3492. 


\section{$N, N^{\prime}$-Bis $(2$ '-hydroxy-1'-naphthylidene)-[1R-(1 $\left.\alpha, 2 \alpha, 5 \alpha)\right]-2$-amino-7,7-dimethyl-2-}

bicyclo[3.3.1]heptane-ethanamine (1c). General Procedure A was followed, from diamine 1 (98 $\mathrm{mg}$, $0.58 \mathrm{mmol}$ ) and 2-hydroxy-1-naphthaldehyde (201 mg, $1.16 \mathrm{mmol})$, with heating at $75{ }^{\circ} \mathrm{C}$ for $5 \mathrm{~h}$. Filtration, followed by recrystallization from absolute ethanol provided 1c $(230 \mathrm{mg}, 84 \%)$ : $[\alpha]^{19}{ }_{\mathrm{D}}-58.4$ (c 2.92, $\mathrm{CH}_{2} \mathrm{Cl}_{2}$ ); ${ }^{1} \mathrm{H}$ NMR (400 MHz, $\left.\mathrm{CDCl}_{3}\right) \delta 1.21$ (d, J=10.3 Hz, 1H), $1.24(\mathrm{~s}, 3 \mathrm{H}), 1.43$ (s, 3H ), 2.07 (app d, $J=6.5 \mathrm{~Hz}, 3 \mathrm{H}), 2.20-2.33(\mathrm{~m}, 2 \mathrm{H}), 2.39-2.44(\mathrm{~m}, 1 \mathrm{H}), 2.61(\operatorname{app~t}, J=5 \mathrm{~Hz}, 1 \mathrm{H}), 3.83$ (d, $J=13 \mathrm{~Hz}, 1 \mathrm{H}), 3.92(\mathrm{~d}, J=13.0 \mathrm{~Hz}, 1 \mathrm{H}), 6.74(\mathrm{~d}, J=9 \mathrm{~Hz}, 1 \mathrm{H}), 6.85(\mathrm{~d}, J=9 \mathrm{~Hz}, 1 \mathrm{H}), 7.04(\operatorname{app~t}, J$ $=7.5 \mathrm{~Hz}, 1 \mathrm{H}), 7.11(\operatorname{app~t}, J=7.4 \mathrm{~Hz}, 1 \mathrm{H}), 7.17(\operatorname{app~t}, J=7.5 \mathrm{~Hz}, 1 \mathrm{H}), 7.21(\mathrm{dt}, J=8,1 \mathrm{~Hz}, 1 \mathrm{H})$, $7.36(\mathrm{~d}, J=8 \mathrm{~Hz}, 1 \mathrm{H}), 7.44(\mathrm{~d}, J=7 \mathrm{~Hz}, 1 \mathrm{H}), 7.45(\mathrm{~d}, J=9 \mathrm{~Hz}, 1 \mathrm{H}), 7.53(\mathrm{~d}, J=9 \mathrm{~Hz}, 2 \mathrm{H}), 7.58(\mathrm{~d}, J$ $=8 \mathrm{~Hz}, 1 \mathrm{H}), 8.46(\mathrm{~s}, 1 \mathrm{H}), 8.61(\mathrm{~s}, 1 \mathrm{H}), 14.69(\mathrm{br} \mathrm{s}, 2 \mathrm{H}) ;{ }^{13} \mathrm{C} \mathrm{NMR}\left(100 \mathrm{MHz}, \mathrm{CDCl}_{3}\right) \delta 23.7,25.2$, 27.3, 28.1, 28.7, 38.4, 40.2, 47.0, 64.5, 65.5, 106.3, 107.4, 117.7, 118.2, 122.5, 122.9, 122.94, 124.8, 126.0, 126.5, 127.8, 127.9, 128.8, 133.0, 133.7, 136.9, 147.6, 154.9, 161.2, 172.9, 177.2; HRMS (FAB, 3-NOBA) calcd for $\mathrm{C}_{32} \mathrm{H}_{33} \mathrm{~N}_{2} \mathrm{O}_{2}(\mathrm{M}+\mathrm{H})^{+}$477.2542, obsd 477.2530.

\section{$N, N^{\prime}$-Bis(1'-hydroxy-2'-naphthylidene)-[1R-(1 $\left.\left.\alpha, 2 \alpha, 5 \alpha\right)\right]$-2-amino-7,7-dimethyl-2-}

bicyclo[3.3.1] heptane-ethanamine (1d). Following General Procedure A, from diamine 1 (87.5 mg, $0.52 \mathrm{mmol}$ ) and 1-hydroxy-2-naphthaldehyde (179 $\mathrm{mg}, 1.04 \mathrm{mmol})$, heating at reflux overnight gave crude salen as an oil. $\mathrm{SiO}_{2}$ column chromatography $(0 \% \rightarrow 30 \%$ EtOAc-hexanes $)$ afforded pure salen 1d (185 mg, 73\%): $[\alpha]^{19}{ }_{\mathrm{D}}^{-355}\left(c 0.81, \mathrm{CH}_{2} \mathrm{Cl}_{2}\right) ;{ }^{1} \mathrm{H} \mathrm{NMR}\left(400 \mathrm{MHz}, \mathrm{CDCl}_{3}\right) \delta 1.24(\mathrm{~d}, J=10.3$ $\mathrm{Hz}, 1 \mathrm{H}), 1.26(\mathrm{~s}, 3 \mathrm{H}), 1.45(\mathrm{~s}, 3 \mathrm{H}) 2.09(\mathrm{app} \mathrm{d}, 3 \mathrm{H}), 2.21-2.33(\mathrm{~m}, 2 \mathrm{H}), 2.44(\mathrm{~m}, J=5,4.7 \mathrm{~Hz}, 1 \mathrm{H})$, $3.81(\mathrm{~d}, J=13 \mathrm{~Hz}, 1 \mathrm{H}), 3.86(\mathrm{~d}, J=13 \mathrm{~Hz}, 1 \mathrm{H}), 6.68(\mathrm{~d}, J=9.8 \mathrm{~Hz}, 1 \mathrm{H}), 6.73(\mathrm{~d}, J=9.8 \mathrm{~Hz}, 1 \mathrm{H}), 6.78$ $(\mathrm{d}, J=9.8 \mathrm{~Hz}, 1 \mathrm{H}), 6.83(\mathrm{~d}, J=9.8 \mathrm{~Hz}, 1 \mathrm{H}), 7.39-7.62(\mathrm{~m}, 6 \mathrm{H}), 7.67(\mathrm{~d}, J=10 \mathrm{~Hz}, 1 \mathrm{H}), 7.81(\mathrm{~s}, 1 \mathrm{H})$, $8.43(\mathrm{~s}, 1 \mathrm{H}), 8.45(\mathrm{~s}, 1 \mathrm{H}), 13.93(\mathrm{~s}, 1 \mathrm{H}), 14.02(\mathrm{~d}, J=10 \mathrm{~Hz}, 1 \mathrm{H}) ;{ }^{13} \mathrm{C} \mathrm{NMR}\left(100 \mathrm{MHz}, \mathrm{CDCl}_{3}\right) \delta 23.7$, 25.1, 26.9, 28.0, 28.6, 38.3, 40.1, 46.9, 63.7, 65.0, 108.6, 109.8, 114.7, 116.1, 124.8, 125, 125.2, 127.26, 127.34, 127.4, 128.2, 129.8, 129.9, 130.3, 137, 137.6, 158.4, 164.3, 172.2, 177.1; HRMS (FAB, 3NOBA) calcd for $\mathrm{C}_{32} \mathrm{H}_{33} \mathrm{O}_{2} \mathrm{~N}_{2}(\mathrm{M}+\mathrm{H})^{+} 477.2542$, obsd 477.2531.

\section{$N, N^{\prime}$-Bis(3',5'-diiodosalicylidene)-[1R-(1 $\left.\left.\alpha, 2 \alpha, 5 \alpha\right)\right]$-2-amino-7,7-dimethyl-2-bicyclo[3.3.1]heptane-}

ethanamine (1e). The title salen was obtained, following General Procedure A, from diamine 1 (36.4 $\mathrm{mg}, 0.22 \mathrm{mmol})$ and 3,5-diiodosalicylaldehyde $(164 \mathrm{mg}, 0.44 \mathrm{mmol})$, with heating at 40-50 ${ }^{\circ} \mathrm{C}$ for $5 \mathrm{~h}$. Recrystallization from ethanol at $-20{ }^{\circ} \mathrm{C}$ afforded pure 1 e $(136 \mathrm{mg}, 91 \%):\left[\alpha{ }^{19} \mathrm{D}-24.8(c) 3.05\right.$, $\mathrm{CH}_{2} \mathrm{Cl}_{2}$ ); ${ }^{1} \mathrm{H}$ NMR $\left(400 \mathrm{MHz}, \mathrm{CD}_{2} \mathrm{Cl}_{2}\right) \delta 0.98(\mathrm{~d}, J=10 \mathrm{~Hz}, 1 \mathrm{H}), 1.20(\mathrm{~s}, 3 \mathrm{H}), 1.36(\mathrm{~s}, 3 \mathrm{H}), 1.91-$ $2.42(\mathrm{~m}, 7 \mathrm{H}), 3.84(\mathrm{~d}, J=13 \mathrm{~Hz}, 1 \mathrm{H}), 3.92(\mathrm{~d}, J=13 \mathrm{~Hz}, 1 \mathrm{H}), 7.44(\mathrm{~s}, 1 \mathrm{H}), 7.48(\mathrm{~s}, 1 \mathrm{H}), 7.92(\mathrm{~s}, 1 \mathrm{H})$, 8.02 (s, 3H), 14.32 (br s, $1 \mathrm{H}), 15.31$ (br s, $1 \mathrm{H}),{ }^{13} \mathrm{C} \mathrm{NMR}\left(100 \mathrm{MHz}, \mathrm{CD}_{2} \mathrm{Cl}_{2}\right) \delta 23.9,25.8,26.2,28.3$, 28.9, 38.9, 40.5, 48.2, 67.9, 67.8, 77.6, 79.6, 87.9, 90.2, 119.2, 120.2, 140.6, 141.1, 149.3, 149.4, 160.6, 161.4, 164.0, 165.8; HRMS (FAB, 3-NOBA) calcd for $\mathrm{C}_{24} \mathrm{H}_{25} \mathrm{~N}_{2} \mathrm{O}_{2} \mathrm{I}_{4}(\mathrm{M}+\mathrm{H})^{+} 880.8095$, obsd 880.8121 . 


\section{$N, N^{\prime}$-Bis(4'-benzyloxysalicylidene)-[1R-(1 $\left.\left.\alpha, 2 \alpha, 5 \alpha\right)\right]$-2-amino-7,7-dimethyl-2-}

bicyclo[3.3.1] heptane-ethanamine (1f). The target salen was obtained following General Procedure A, from diamine $1(167 \mathrm{mg}, 0.54 \mathrm{mmol})$ and 4-benzyloxysalicylaldehyde (246 mg, $1.08 \mathrm{mmol})$, after heating at $40-50{ }^{\circ} \mathrm{C}$ for $5 \mathrm{~h}$. Recrystallization from ethanol yielded pure salen $1 \mathrm{f}(105 \mathrm{mg}$; $100 \%)$ : $[\alpha]^{19}{ }_{\mathrm{D}}-16.1\left(c \mathrm{c} .93, \mathrm{CH}_{2} \mathrm{Cl}_{2}\right) ;{ }^{1} \mathrm{H}$ NMR $\left(400 \mathrm{MHz}, \mathrm{CDCl}_{3}\right) \delta 1.08(\mathrm{~d}, J=10.4 \mathrm{~Hz}, 1 \mathrm{H}), 1.19(\mathrm{~s}, 3 \mathrm{H})$, $1.35(\mathrm{~s}, 3 \mathrm{H}), 1.91-2.04(\mathrm{~m}, 3 \mathrm{H}), 2.08-2.22(\mathrm{~m}, 2 \mathrm{H}), 2.28-2.31(\mathrm{~m}, 1 \mathrm{H}), 2.39$ (app t, $J=5 \mathrm{~Hz}, 1 \mathrm{H}), 3.79$ (d, $J=14 \mathrm{~Hz}, 1 \mathrm{H}), 3.81(\mathrm{~d}, J=12 \mathrm{~Hz}, 1 \mathrm{H}), 5.03(\mathrm{~s}, 3 \mathrm{H}), 5.04(\mathrm{~s}, 3 \mathrm{H}), 6.33(\mathrm{dd}, J=9,2 \mathrm{~Hz}, 1 \mathrm{H}), 6.40$ (s, 1H), 6.34-6.42 (m, 2H), $6.48(\mathrm{~d}, J=2 \mathrm{~Hz}, 1 \mathrm{H}), 6.98(\operatorname{app} \mathrm{t}, J=9 \mathrm{~Hz}, 2 \mathrm{H}), 7.30-7.42(\mathrm{~m}, 10 \mathrm{H}), 7.90$ (s, 1H), 8.02 (s, 1H), 13.7 (br s, 1H), 14.61(br s, 1H); ${ }^{13} \mathrm{C} \mathrm{NMR} \mathrm{(100} \mathrm{MHz,} \mathrm{CDCl}_{3}$ ) $\delta$ 23.7, 25.4, 26.1, 28.0, 28.2, 28.4, 38.2, 39.9, 47.6, 66.3, 67.6, 69.8, 69.9, 102.0, 102.5, 106.7, 107, 111.7, 112.3, 127.5, $128,128.1,128.5,128.6,132.8,133.3,136.3,136.4,160.1,162.7,163.3,164.9,165.8,168.6$; HRMS (FAB, 3-NOBA) calcd for $\mathrm{C}_{38} \mathrm{H}_{41} \mathrm{O}_{4} \mathrm{~N}_{2}(\mathrm{M}+\mathrm{H})^{+}$589.3066, obsd 589.3047.

\section{$N, N^{\prime}$-Bis(8'-hydroxy-1',1',7',7'-tetramethyljulolidine-9'-methylidene)-[1R-(1 $\left.\left.\alpha, 2 \alpha, 5 \alpha\right)\right]-2$-amino-}

7,7-dimethyl-2-bicyclo[3.3.1]heptane-ethanamine (1g). Following General Procedure A, from diamine 1 (55 mg, $0.33 \mathrm{mmol}$ ) and 8-hydroxy-1,1,7,7-tetramethyljulolidine-9-carboxaldehyde (180 $\mathrm{mg}$, $0.66 \mathrm{mmol})$, was obtained crude salen after stirring at $\mathrm{rt}$ for $24 \mathrm{~h}$. Column chromatography $\left(2 \% \mathrm{Et}_{3} \mathrm{~N}-\right.$ hexanes) afforded pure $\left.1 \mathrm{~g}(130 \mathrm{mg}, 65 \%):[\alpha]^{19}{ }_{\mathrm{D}}+63.6(c) 3.55, \mathrm{CH}_{2} \mathrm{Cl}_{2}\right) ;{ }^{1} \mathrm{H}$ NMR $(400 \mathrm{MHz}$, $\left.\mathrm{CDCl}_{3}\right) \delta 1.18(\mathrm{~s}, 4 \mathrm{H}), 1.21(\mathrm{~s}, 12 \mathrm{H}), 1.32(\mathrm{~s}, 3 \mathrm{H}) 1.49(\mathrm{~s}, 12 \mathrm{H}), 1.69-1.74(\mathrm{~m}, 8 \mathrm{H}), 1.93-1.97(\mathrm{~m}, 3 \mathrm{H})$, 2.35 (app d, $J=4 \mathrm{~Hz}, 1 \mathrm{H}), 3.09$ (br s, 4H), 3.17 (app t, $J=6 \mathrm{~Hz}, 4 \mathrm{H}), 3.58(\mathrm{~d}, J=12 \mathrm{~Hz}, 1 \mathrm{H}), 3.75$ (d, $J=12 \mathrm{~Hz}, 1 \mathrm{H}), 6.76(\mathrm{~s}, 1 \mathrm{H}), 6.77(\mathrm{~s}, 1 \mathrm{H}), 7.93(\mathrm{~s}, 1 \mathrm{H}), 7.97(\mathrm{~s}, 1 \mathrm{H}), 13.91(\mathrm{br} \mathrm{s}, 1 \mathrm{H}), 14.63(\mathrm{br} \mathrm{s}, 1 \mathrm{H})$, ${ }^{13} \mathrm{C}$ NMR (100 MHz, $\left.\mathrm{CDCl}_{3}\right) \delta$ 23.9, 25.8, 26.1, 28.2, 28.6, 28.7, 31.2, 31.3, 31.7, 32.2, 32.3, 36.7, $36.8,38.3,40.2$, 40.2, 40.3, 46.3, 47.0, 47.1, 47.4, 47.5, 48.6, 65.8, 68.2, 108.9, 108.93, 114.8, 114.9, $127.7,128.1,145.7,145.9,160.5,161.0,163.7,166.0$; HRMS (FAB, 3-NOBA) $\mathrm{C}_{44} \mathrm{H}_{63} \mathrm{O}_{2} \mathrm{~N}_{4}(\mathrm{M}+\mathrm{H})^{+}$ 679.4951, obsd 679.4974.

$N, N^{\prime}$-Bis(3',5'-di-tert-butylsalicylidene)-(1S)-phenyl-1,2-ethylenediamine (2a). (1S)-Phenyl-1,2ethylenediamine $\mathbf{2}^{2,3}$ was prepared from $L-(+)-\alpha$-phenylglycine (Janssen Chimica, 99\%). Following General Procedure A, from (1S)-phenyl-1,2-ethylenediamine $(250 \mathrm{mg}, 1.83 \mathrm{mmol})$ and 3,5-di-tertbutylsalicylaldehyde ( $861 \mathrm{mg}, 3.67 \mathrm{mmol}$ ), after stirring at $45^{\circ} \mathrm{C}$ for $24 \mathrm{~h}$, was obtained 2a. Trituration with ethanol provided pure salen $(823 \mathrm{mg}, 79 \%)$ : $[\alpha]^{19}{ }_{\mathrm{D}}+113.0\left(\right.$ c $\left.1.29, \mathrm{CH}_{2} \mathrm{Cl}_{2}\right) ;{ }^{1} \mathrm{H}$ NMR $(400$

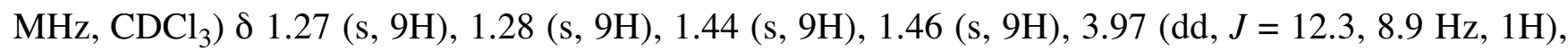
$4.15(\mathrm{dd}, J=12.3,4.1 \mathrm{~Hz}, 1 \mathrm{H}), 4.71(\mathrm{dd}, J=8.9,4.1 \mathrm{~Hz}, 1 \mathrm{H}), 7.04(\mathrm{~d}, J=2.5 \mathrm{~Hz}, 1 \mathrm{H}), 7.05(\mathrm{~d}, J=2.5$ $\mathrm{Hz}, 1 \mathrm{H}), 7.32(\mathrm{t}, J=7.3 \mathrm{~Hz}, 1 \mathrm{H}), 7.36(\mathrm{~d}, J=2.5 \mathrm{~Hz}, 1 \mathrm{H}), 7.37(\mathrm{~d}, J=2.5 \mathrm{~Hz}, 1 \mathrm{H}), 7.39$ (d, $J=7.3 \mathrm{~Hz}$, $1 \mathrm{H}), 7.41(\mathrm{~d}, J=7.3 \mathrm{~Hz}, 1 \mathrm{H}), 7.48(\mathrm{~d}, J=7.3 \mathrm{~Hz}, 2 \mathrm{H}), 8.35$ (s, 1H), $8.44(\mathrm{~s}, 1 \mathrm{H}), 13.59$ (br s, 1H), 13.62 (br s, $1 \mathrm{H}) ;{ }^{13} \mathrm{C}$ NMR $\left(100 \mathrm{MHz}, \mathrm{CDCl}_{3}\right) \delta 29.40,29.44,31.42,31.44,34.1,35.0,66.5,73.9$, 117.76, 117.83, 126.1, 126.4, 127.1, 127.2, 127.7, 128.8, 136.5, 136.6, 140.0, 140.1, 140.9, 157.9, 158.1, 167.1, 167.9; HRMS (FAB, 3-NOBA) calcd for $\mathrm{C}_{38} \mathrm{H}_{53} \mathrm{O}_{2} \mathrm{~N}_{2}(\mathrm{M}+\mathrm{H})^{+} 569.4108$, obsd 569.4091. 
$N, N^{\prime}$-Bis(3'-tert-butylsalicylidene)-(1S)-phenyl-1,2-ethylenediamine (2b). The title salen was obtained from (1S)-phenyl-1,2-ethylenediamine $(219 \mathrm{mg}, 1.61 \mathrm{mmol})$ and 3-tert-butylsalicylaldehyde (516 mg, $2.89 \mathrm{mmol}$ ), following General Procedure A, with stirring at $45^{\circ} \mathrm{C}$ for $72 \mathrm{~h}$. Following filtration, trituration with ice-cold ethanol provided pure $2 \mathbf{b}(515 \mathrm{mg}, 78 \%):[\alpha]{ }_{\mathrm{D}}^{19}+43.9(c) 1.03$, $\mathrm{CH}_{2} \mathrm{Cl}_{2}$ ); ${ }^{1} \mathrm{H} \mathrm{NMR}\left(400 \mathrm{MHz}, \mathrm{CDCl}_{3}\right) \delta 1.40(\mathrm{~s}, 9 \mathrm{H}), 1.43(\mathrm{~s}, 9 \mathrm{H}), 3.97(\mathrm{dd}, J=12.3,8.6 \mathrm{~Hz}, 1 \mathrm{H})$, $4.13(\mathrm{dd}, J=12.3,4.5 \mathrm{~Hz}, 1 \mathrm{H}), 4.70(\mathrm{dd}, J=8.6,4.5 \mathrm{~Hz}, 1 \mathrm{H}), 6.75(\mathrm{dd}, J=7.6,2.9 \mathrm{~Hz}, 1 \mathrm{H}), 6.77$ (dd, $J=7.6,2.9 \mathrm{~Hz}, 1 \mathrm{H}), 7.03(\mathrm{dd}, J=7.6,1.5 \mathrm{~Hz}, 1 \mathrm{H}), 7.06(\mathrm{dd}, J=7.6,1.5 \mathrm{~Hz}, 1 \mathrm{H}), 7.27-7.32(\mathrm{~m}, 3 \mathrm{H})$, $7.40(\mathrm{t}, J=7.5 \mathrm{~Hz}, 2 \mathrm{H}), 7.46(\mathrm{~d}, J=7.3 \mathrm{~Hz}, 2 \mathrm{H}), 8.29$ (s, 1H), 8.42 (s, 1H), 13.74 (br s, 1H), 13.76 (br s, 1H); ${ }^{13} \mathrm{C}$ NMR $\left(100 \mathrm{MHz}, \mathrm{CDCl}_{3}\right) \delta 29.28,29.34,34.8,66.3,73.9,117.8,118.0,118.5,118.6$, $127.1,127.8,128.8,129.5,129.7,129.9,130.2$, 137.2, 137.3, 140.6, 160.2, 160.4, 166.6, 167.5; HRMS (FAB, 3-NOBA) calcd for $\mathrm{C}_{30} \mathrm{H}_{37} \mathrm{O}_{2} \mathrm{~N}_{2}(\mathrm{M}+\mathrm{H})+457.2856$, obsd 457.2841.

$N, N^{\prime}$-Bis(2'-hydroxy-1'-naphthylidene)-(1S)-phenyl-1,2-ethylenediamine (2c). General Procedure A was followed to obtain the title compound from (1S)-phenyl-1,2-ethylenediamine (253 mg, 1.86 mmol) and 2-hydroxy-1-naphthaldehyde (576 mg, $3.35 \mathrm{mmol})$, after stirring at $45{ }^{\circ} \mathrm{C}$ for $10 \mathrm{~h}$. Trituration with cold ethanol afforded the pure salen $2 \mathrm{c}(689 \mathrm{mg}, 93 \%)$ : $[\alpha]{ }^{19}{ }_{\mathrm{D}}+34.4\left(c 1.02, \mathrm{CH}_{2} \mathrm{Cl}_{2}\right)$; ${ }^{1} \mathrm{H} \mathrm{NMR}\left(400 \mathrm{MHz} \mathrm{CDCl}_{3}\right) \delta 4.03(\mathrm{dd}, J=12.9,8.3 \mathrm{~Hz}, 1 \mathrm{H}), 4.21(\mathrm{dd}, J=12.9,4.5 \mathrm{~Hz}, 1 \mathrm{H}), 4.84$ $(\mathrm{dd}, J=8.3,4.5 \mathrm{~Hz}, 1 \mathrm{H}), 6.90(\mathrm{~d}, J=9.2 \mathrm{~Hz}, 1 \mathrm{H}), 7.04(\mathrm{~d}, J=9.1 \mathrm{~Hz}, 1 \mathrm{H}), 7.17-7.25(\mathrm{~m}, 2 \mathrm{H})$, $7.32-7.45(\mathrm{~m}, 5 \mathrm{H}), 7.50-7.55(\mathrm{~m}, 3 \mathrm{H}), 7.61(\mathrm{~d}, J=8.9 \mathrm{~Hz}, 2 \mathrm{H}), 7.69(\mathrm{~d}, J=9.1 \mathrm{~Hz}, 1 \mathrm{H}), 7.76(\mathrm{~d}, J=$ $8.4 \mathrm{~Hz}, 1 \mathrm{H}), 7.88(\mathrm{~d}, J=8.4 \mathrm{~Hz}, 1 \mathrm{H}), 8.77(\mathrm{~s}, 1 \mathrm{H}), 9.12(\mathrm{~s}, 1 \mathrm{H}), 14.60$ (br s, $1 \mathrm{H}), 15.15(\mathrm{br} \mathrm{s}, 1 \mathrm{H}) ;{ }^{13} \mathrm{C}$ $\operatorname{NMR}\left(100 \mathrm{MHz}, \mathrm{CDCl}_{3}\right) \delta 62.1,72.1,107.4,108.1,118.4,118.9,121.1,123.0,123.2,126.6,126.9$, 127.2, 127.79, 127.83, 128.4, 129.2, 132.8, 133.2, 135.7, 136.6, 139.2, 160.5, 161.2, 167.4, 172.2; HRMS (FAB, 3-NOBA) calcd for $\mathrm{C}_{30} \mathrm{H}_{25} \mathrm{~N}_{2} \mathrm{O}_{2}(\mathrm{M}+\mathrm{H})^{+} 445.1917$, obsd 445.1905.

$N, N^{\prime}$-Bis(1'-hydroxy-2'-naphthylidene)-(1S)-phenyl-1,2-ethylenediamine (2d). Salen $2 \mathrm{~d}$ was accessed, following General Procedure A, from (1S)-phenyl-1,2-ethylenediamine (197 mg, $1.45 \mathrm{mmol}$ ) and 1-hydroxy-2-naphthaldehyde (498 mg, $3.89 \mathrm{mmol})$, with stirring at $45^{\circ} \mathrm{C}$ for $16 \mathrm{~h}$. Purification was achieved by trituration with cold ethanol to give pure $2 \mathbf{d}(542 \mathrm{mg}, 84 \%):[\alpha]^{19} \mathrm{D}+688(c 0.75$, $\left.\mathrm{CH}_{2} \mathrm{Cl}_{2}\right) ;{ }^{1} \mathrm{H} \mathrm{NMR}\left(400 \mathrm{MHz}, \mathrm{CDCl}_{3}\right) \delta 3.96(\mathrm{dd}, J=13.1,8.4 \mathrm{~Hz}, 1 \mathrm{H}), 4.11(\mathrm{dd}, J=13.1,4.6 \mathrm{~Hz}$, $1 \mathrm{H}), 4.75(\mathrm{dd}, J=8.4,4.6 \mathrm{~Hz}, 1 \mathrm{H}), 6.84(\mathrm{~d}, J=8.8 \mathrm{~Hz}, 1 \mathrm{H}), 6.89$ (d, $J=8.8 \mathrm{~Hz}, 1 \mathrm{H}), 7.04(\mathrm{~s}, 2 \mathrm{H})$, $7.33-7.37(\mathrm{~m}, 1 \mathrm{H}), 7.40-7.58(\mathrm{~m}, 9 \mathrm{H}), 7.65(\mathrm{~d}, J=7.9 \mathrm{~Hz}, 1 \mathrm{H}), 7.88(\mathrm{~s}, 1 \mathrm{H}), 8.25(\mathrm{~s}, 1 \mathrm{H}), 8.39(\mathrm{~d}, J=$ $8.3 \mathrm{~Hz}, 1 \mathrm{H}), 8.42$ (d, $J=8.8 \mathrm{~Hz}, 1 \mathrm{H}), 13.82$ (br s, $1 \mathrm{H}), 14.46$ (br s, $1 \mathrm{H}) ;{ }^{13} \mathrm{C} \mathrm{NMR}\left(100 \mathrm{MHz}, \mathrm{CDCl}_{3}\right)$

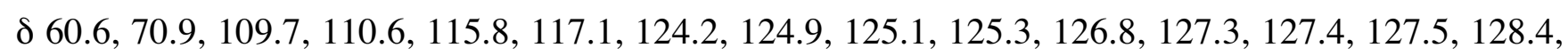
129.1, 129.3, 129.9, 136.4, 137.0, 138.8, 163.5, 164.5, 166.7, 172.3; HRMS (FAB, 3-NOBA) calcd for $\mathrm{C}_{30} \mathrm{H}_{25} \mathrm{~N}_{2} \mathrm{O}_{2}(\mathrm{M}+\mathrm{H})^{+}$445.1917, obsd 445.1925. 
$N, N^{\prime}$-Bis(3',5'-diiodosalicylidene)-(1S)-phenyl-1,2-ethylenediamine (2e). The title salen was available from $(1 S)$-phenyl-1,2-ethylenediamine $(105 \mathrm{mg}, 0.77 \mathrm{mmol})$ and 3,5-diiodosalicylaldehyde (520 mg, $1.38 \mathrm{mmol}$ ), following General Procedure A, with stirring at $45^{\circ} \mathrm{C}$ for $24 \mathrm{~h}$. Trituration with cold ethanol afforded pure $2 \mathrm{e}(567 \mathrm{mg}, 96 \%)$ : $[\alpha]^{19}{ }_{\mathrm{D}}+46.6\left(c 1.00, \mathrm{CH}_{2} \mathrm{Cl}_{2}\right) ;{ }^{1} \mathrm{H} \mathrm{NMR}(400 \mathrm{MHz}$, $\left.\mathrm{CDCl}_{3}\right) \delta 3.97(\mathrm{dd}, J=12.5,8.7 \mathrm{~Hz}, 1 \mathrm{H}), 4.17(\mathrm{dd}, J=12.5,4.3 \mathrm{~Hz}, 1 \mathrm{H}), 4.72(\mathrm{dd}, J=8.7,4.3 \mathrm{~Hz}$, $1 \mathrm{H}), 7.31-7.41(\mathrm{~m}, 5 \mathrm{H}), 7.44(\mathrm{~d}, J=2.0 \mathrm{~Hz}, 1 \mathrm{H}), 7.48(\mathrm{~d}, J=2.0 \mathrm{~Hz}, 1 \mathrm{H}), 8.02(\mathrm{~d}, J=2.0 \mathrm{~Hz}, 1 \mathrm{H})$, $8.03(\mathrm{~d}, J=2.0 \mathrm{~Hz}, 1 \mathrm{H}), 8.05(\mathrm{~s}, 1 \mathrm{H}), 8.18(\mathrm{~s}, 1 \mathrm{H}), 14.25(\mathrm{br} \mathrm{s}, 1 \mathrm{H}), 14.36(\mathrm{br} \mathrm{s}, 1 \mathrm{H}) ;{ }^{13} \mathrm{C}$ NMR $(100$ $\left.\mathrm{MHz}, \mathrm{CDCl}_{3}\right) \delta$ 65.3, 73.2, 79.5, 79.9, 87.0, 87.5, 119.6, 119.8, 126.8, 128.4, 129.1, 138.9, 140.0, 140.2, 148.9, 160.0, 160.5, 164.0, 165.0; HRMS (FAB, 3-NOBA) calcd for $\mathrm{C}_{22} \mathrm{H}_{17} \mathrm{O}_{2} \mathrm{~N}_{2} \mathrm{I}_{4}(\mathrm{M}+\mathrm{H})^{+}$ 848.7470 , obsd 848.7496 .

$N, N^{\prime}$-Bis(4'-benzyloxysalicylidene)-(1S)-phenyl-1,2-ethylenediamine (2f). From (1S)-phenyl-1,2ethylenediamine (212 mg, $1.56 \mathrm{mmol}$ ) and 4-benzyloxy-2-hydroxybenzaldehyde (630 mg, $2.76 \mathrm{mmol}$ ), following General Procedure A, with stirring at $45{ }^{\circ} \mathrm{C}$ for $24 \mathrm{~h}$, was obtained salen 2f. Purification was achieved by trituration with cold ethanol, yielding pure $2 \mathbf{f}(710 \mathrm{mg}, 92 \%):[\alpha]^{19}{ }_{\mathrm{D}}+119(c 0.96$, $\mathrm{CH}_{2} \mathrm{Cl}_{2}$ ); ${ }^{1} \mathrm{H}$ NMR $\left(400 \mathrm{MHz}, \mathrm{CDCl}_{3}\right) \delta 3.88(\mathrm{dd}, J=12.4,8.3 \mathrm{~Hz}, 1 \mathrm{H}), 4.06(\mathrm{dd}, J=12.4,4.3 \mathrm{~Hz}$, $1 \mathrm{H}), 4.61(\mathrm{dd}, J=8.3,4.3 \mathrm{~Hz}, 1 \mathrm{H}), 5.05(\mathrm{~d}, J=5.2 \mathrm{~Hz}, 4 \mathrm{H}), 6.44-6.56(\mathrm{~m}, 4 \mathrm{H}), 7.06(\mathrm{~d}, J=8.6 \mathrm{~Hz}$, $1 \mathrm{H}), 7.10(\mathrm{~d}, J=8.6 \mathrm{~Hz}, 1 \mathrm{H}), 7.30-7.45(\mathrm{~m}, 15 \mathrm{H}), 8.12(\mathrm{~s}, 1 \mathrm{H}), 8.28(\mathrm{~s}, 1 \mathrm{H}), 13.65$ (br s, 1H), 13.78 (br s, $1 \mathrm{H}) ;{ }^{13} \mathrm{C} \mathrm{NMR}\left(100 \mathrm{MHz}, \mathrm{CDCl}_{3}\right) \delta 65.7,69.87,69.90,73.4,101.96,101.99,106.98,107.04,112.4$, 112.6, 126.9, 127.43, 127.44, 127.7, 128.0, 128.5, 128.8, 132.8, 133.0, 136.32, 136.33, 140.6, 162.49, 162.54, 163.5, 164.4, 164.9, 165.6; HRMS (FAB, 3-NOBA) calcd for $\mathrm{C}_{36} \mathrm{H}_{33} \mathrm{~N}_{2} \mathrm{O}_{4}(\mathrm{M}+\mathrm{H})^{+}$557.2441, obsd 557.2434 .

\section{$N, N^{\prime}$-Bis(8'-hydroxy-1',1',7',7'-tetramethyljulolidinyl-9'-methylidene)-(1S)-phenyl-1,2-}

ethylenediamine $\mathbf{( 2 g )}$. Salen $\mathbf{2 g}$ was obtained from $(1 S)$-phenyl-1,2-ethylenediamine (126 mg, 0.93 mmol) and 8-hydroxy-1,1,7,7-tetramethyljulolidinyl-9-carboxaldehyde (455 mg, $1.67 \mathrm{mmol}$ ), following General Procedure A, after stirring at $45^{\circ} \mathrm{C}$ for $24 \mathrm{~h}$, filtration, and washing with cold ethanol. Subsequent recrystallization from ethanol afforded the pure salen $(304 \mathrm{mg}, 57 \%):[\alpha]^{19}{ }_{\mathrm{D}}+272(c 0.91$, $\left.\mathrm{CH}_{2} \mathrm{Cl}_{2}\right) ;{ }^{1} \mathrm{H}$ NMR (400 MHz, $\left.\mathrm{CDCl}_{3}\right) \delta 1.18(\mathrm{~s}, 9 \mathrm{H}), 1.20(\mathrm{~s}, 3 \mathrm{H}), 1.44(\mathrm{~s}, 3 \mathrm{H}), 1.46(\mathrm{~s}, 3 \mathrm{H}), 1.47(\mathrm{~s}$, $3 \mathrm{H}), 1.49(\mathrm{~s}, 3 \mathrm{H}), 1.68(\mathrm{t}, J=5.5 \mathrm{~Hz}, 4 \mathrm{H}), 1.71-1.75(\mathrm{~m}, 4 \mathrm{H}), 3.09(\mathrm{t}, J=5.5 \mathrm{~Hz}, 4 \mathrm{H}), 3.14-3.18(\mathrm{~m}$, $4 \mathrm{H}), 3.76(\mathrm{dd}, J=12.1,8.5 \mathrm{~Hz}, 1 \mathrm{H}), 3.97(\mathrm{dd}, J=12.1,4.3 \mathrm{~Hz}, 1 \mathrm{H}), 4.52(\mathrm{dd}, J=8.5,4.3 \mathrm{~Hz}, 1 \mathrm{H})$, $6.75(\mathrm{~s}, 1 \mathrm{H}), 6.78(\mathrm{~s}, 1 \mathrm{H}), 7.23-7.26(\mathrm{~m}, 1 \mathrm{H}), 7.34(\mathrm{t}, J=7.5 \mathrm{~Hz}, 2 \mathrm{H}), 7.41(\mathrm{~d}, J=7.5 \mathrm{~Hz}, 2 \mathrm{H}), 7.98(\mathrm{~s}$,

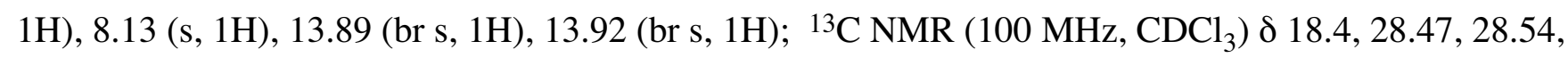
31.1, 31.2, 31.7, 32.2, 36.6, 40.1, 46.95, 46.98, 47.3, 58.4, 65.8, 73.5, 108.7, 108.8, 114.66, 114.73, $121.5,121.7,127.1,127.2,127.6,127.9,128.5,142.0,145.6,145.7,160.0,161.2,165.4,165.7$; HRMS (FAB, 3-NOBA) calcd for $\mathrm{C}_{42} \mathrm{H}_{55} \mathrm{~N}_{4} \mathrm{O}_{2}(\mathrm{M}+\mathrm{H})^{+}$647.4325, obsd 647.4303. 
$N, N^{\prime}$-Bis(3',5'-di-tert-butylsalicylidene)-(1S)-benzyl-1,2-ethylenediamine (3a). Salen 3a was accessed by following General Procedure A, from (1S)-benzyl-1,2-ethylenediamine ${ }^{4}$ (235 mg, 1.57 $\mathrm{mmol}$ ) and 3,5-di-tert-butylsalicylaldehyde (660 mg, $2.82 \mathrm{mmol}, 1.8$ equiv.), with heating at $45^{\circ} \mathrm{C}$ for $24 \mathrm{~h}$. Filtration and trituration (cold ethanol) afforded clean 3a $(486 \mathrm{mg}, 59 \%)$ : $[\alpha]^{19}{ }_{\mathrm{D}}+42.0(c 1.15$, $\mathrm{CH}_{2} \mathrm{Cl}_{2}$ ); ${ }^{1} \mathrm{H}$ NMR (400 MHz, $\left.\mathrm{CDCl}_{3}\right) \delta 1.25$ (s, 9H), 1.28 (s, 9H), 1.43 (s, 9H), 1.45 (s, 9H), 3.02 (dd, $J=13.5,7.4 \mathrm{~Hz}, 1 \mathrm{H}), 3.12(\mathrm{dd}, J=13.5,5.3 \mathrm{~Hz}, 1 \mathrm{H}), 3.75(\mathrm{~m}, 2 \mathrm{H}), 3.95(\mathrm{dd}, J=11.5,2.6 \mathrm{~Hz}, 1 \mathrm{H})$, $6.94(\mathrm{~d}, J=2.2 \mathrm{~Hz}, 1 \mathrm{H}), 7.04(\mathrm{~d}, J=2.2 \mathrm{~Hz}, 1 \mathrm{H}), 7.21-7.31(\mathrm{~m}, 5 \mathrm{H}), 7.38(\mathrm{~s}, 2 \mathrm{H}), 8.14(\mathrm{~s}, 1 \mathrm{H}), 8.34(\mathrm{~s}$,

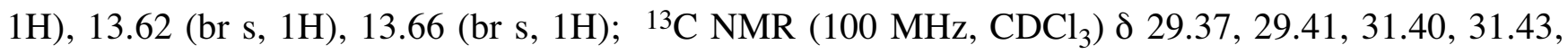
34.04, 34.06, 35.0, 41.0, 63.8, 71.4, 117.6, 117.8, 126.0, 126.1, 126.5, 126.98, 127.00, 128.5, 129.6, $136.4,136.5,138.1,139.9,140.0,157.98,158.04,166.6,167.7$; HRMS (FAB, 3-NOBA) calcd for $\mathrm{C}_{39} \mathrm{H}_{55} \mathrm{O}_{2} \mathrm{~N}_{2}(\mathrm{M}+\mathrm{H})^{+}$583.4264, obsd 583.4278.

$N, N^{\prime}$-Bis(3'-tert-butylsalicylidene)-(1S)-benzyl-1,2-ethylenediamine (3b). To obtain the title compound, General Procedure A was followed, beginning from (1S)-benzyl-1,2-ethylenediamine (251 $\mathrm{mg}, 1.67 \mathrm{mmol}$ ) and 3-tert-butylsalicylaldehyde (536 mg, $3 \mathrm{mmol}, 1.8$ equiv.). After heating for $48 \mathrm{~h}$, at $45{ }^{\circ} \mathrm{C}$, the precipitated was filtered and triturated with cold ethanol to yield pure $\mathbf{3 b}(553 \mathrm{mg}, 78 \%)$ : $[\alpha]^{19}{ }_{\mathrm{D}}+70.4\left(c 1.13, \mathrm{CH}_{2} \mathrm{Cl}_{2}\right) ;{ }^{1} \mathrm{H}$ NMR $\left(400 \mathrm{MHz}, \mathrm{CDCl}_{3}\right) \delta 1.42(\mathrm{~s}, 9 \mathrm{H}), 1.44(\mathrm{~s}, 9 \mathrm{H}), 3.02(\mathrm{dd}, J=$ $13.5,7.5 \mathrm{~Hz}, 1 \mathrm{H}), 3.13(\mathrm{dd}, J=13.5,5.1 \mathrm{~Hz}, 1 \mathrm{H}), 3.71-3.78(\mathrm{~m}, 2 \mathrm{H}), 3.97(\mathrm{br} \mathrm{d}, J=8.6 \mathrm{~Hz}, 1 \mathrm{H}), 6.74$ $(\mathrm{t}, J=15.4,7.7 \mathrm{~Hz}, 1 \mathrm{H}), 6.78(\mathrm{t}, J=15.4,7.7 \mathrm{~Hz}, 1 \mathrm{H}), 6.95(\mathrm{dd}, J=7.5,1.4 \mathrm{~Hz}, 1 \mathrm{H}), 7.06(\mathrm{dd}, J=7.5$, $1.3 \mathrm{~Hz}, 1 \mathrm{H}), 7.19-7.22(\mathrm{~m}, 3 \mathrm{H}), 7.25-7.31(\mathrm{~m}, 4 \mathrm{H}), 8.08(\mathrm{~s}, 1 \mathrm{H}), 8.33$ (s, 1H), 13.79 (br s, 1H), 13.84 (br s, $1 \mathrm{H}) ;{ }^{13} \mathrm{C} \mathrm{NMR}\left(100 \mathrm{MHz}, \mathrm{CDCl}_{3}\right) \delta 29.27,29.31,34.8,40.9,63.8,71.3,117.7,117.8,118.4$, $118.5,126.5,128.5,129.4,129.5,129.6,129.8,137.1,137.3,137.9,160.3,160.4,166.2,167.4$; HRMS (FAB, 3-NOBA) calcd for $\mathrm{C}_{31} \mathrm{H}_{39} \mathrm{O}_{2} \mathrm{~N}_{2}(\mathrm{M}+\mathrm{H})^{+}$471.3012, obsd 471.3018.

$N, N^{\prime}$-Bis(2'-hydroxy-1'-naphthylidene)-(1S)-benzyl-1,2-ethylenediamine (3c). From (1S)-benzyl1,2-ethylenediamine (260 mg, $1.73 \mathrm{mmol})$ and 2-hydroxy-1-naphthaldehyde (537 mg, $3.1 \mathrm{mmol}, 1.8$ equiv.), following General Procedure A, with heating for $20 \mathrm{~h}$ at $45{ }^{\circ} \mathrm{C}$, was obtained salen $3 \mathrm{c}$. Filtration and washing with cold ethanol provided pure salen $(500 \mathrm{mg}, 70 \%):[\alpha]^{19}{ }_{\mathrm{D}}-10.8(c 1.02$, $\mathrm{CH}_{2} \mathrm{Cl}_{2}$ ); ${ }^{1} \mathrm{H}$ NMR (400 MHz, $\mathrm{CDCl}_{3}$ ) $\delta 3.09$ (dd, $\left.J=13.7,8.0 \mathrm{~Hz}, 1 \mathrm{H}\right), 3.19$ (dd, $J=13.7,5.4 \mathrm{~Hz}$, $1 \mathrm{H}), 3.80(\mathrm{dd}, J=12.8,7.9 \mathrm{~Hz}, 1 \mathrm{H}), 3.89-3.95(\mathrm{~m}, 1 \mathrm{H}), 4.06(\mathrm{dd}, J=12.8,3.7 \mathrm{~Hz}, 1 \mathrm{H}), 6.93(\mathrm{~d}, J=9.2$ $\mathrm{Hz}, 1 \mathrm{H}), 6.99(\mathrm{~d}, J=9.2 \mathrm{~Hz}, 1 \mathrm{H}), 7.16-7.27(\mathrm{~m}, 5 \mathrm{H}), 7.28-7.39(\mathrm{~m}, 4 \mathrm{H}), 7.56(\operatorname{app~t}, J=7.1 \mathrm{~Hz}, 2 \mathrm{H})$, $7.63(\mathrm{dd}, J=7.9,1.2 \mathrm{~Hz}, 3 \mathrm{H}), 7.81(\mathrm{~d}, J=8.4 \mathrm{~Hz}, 1 \mathrm{H}), 8.67(\mathrm{~s}, 1 \mathrm{H}), 8.82(\mathrm{~s}, 1 \mathrm{H}), 14.62$ (br s, $1 \mathrm{H})$, 14.78 (br s, $1 \mathrm{H}) ;{ }^{13} \mathrm{C}$ NMR $\left(100 \mathrm{MHz}, \mathrm{CDCl}_{3}\right) \delta 40.4,59.4,68.8,107.4,107.5,118.3,118.6,121.7$, $122.90,122.92,122.95,126.6,126.9,127.0,127.6,127.8,128.7,128.9,129.0,129.5,132.9,133.1$, 135.7, 136.5, 136.8, 160.45, 160.50, 168.9, 172.2; HRMS (FAB, 3-NOBA) calcd for $\mathrm{C}_{31} \mathrm{H}_{27} \mathrm{~N}_{2} \mathrm{O}_{2}$ $(\mathrm{M}+\mathrm{H})^{+}$459.2073, obsd 459.2060. 
$N, N^{\prime}$-Bis(1'-hydroxy-2'-naphthylidene)-(1S)-benzyl-1,2-ethylenediamine (3d). From (1S)-benzyl1,2-ethylenediamine (266 mg, $1.77 \mathrm{mmol}$ ) and 1-hydroxy-2-naphthaldehyde (549 mg, $3.19 \mathrm{mmol}, 1.8$ equiv.), following General Procedure A, with heating for $10 \mathrm{~h}$ at $45{ }^{\circ} \mathrm{C}$, was obtained 3d. Filtration, followed by washing with cold ethanol provided the pure product $(630 \mathrm{mg}, 86 \%):[\alpha]^{19}{ }_{\mathrm{D}}+816(c 0.23$, $\left.\mathrm{CH}_{2} \mathrm{Cl}_{2}\right)$; ${ }^{1} \mathrm{H}$ NMR (400 MHz, $\left.\mathrm{CDCl}_{3}\right) \delta 3.05(\mathrm{dd}, J=13.7,8.1 \mathrm{~Hz}, 1 \mathrm{H}), 3.15(\mathrm{dd}, J=13.7,5.4 \mathrm{~Hz}$, $1 \mathrm{H}), 3.71(\mathrm{dd}, J=12.8,8.0 \mathrm{~Hz}, 1 \mathrm{H}), 3.77-3.83(\mathrm{~m}, 1 \mathrm{H}), 3.95(\mathrm{dd}, J=12.8,3.5 \mathrm{~Hz}, 1 \mathrm{H}), 6.89(\mathrm{dd}, J=$ 8.6, 6.2 Hz, 2H), 6.93 (dd, $J=8.7,4.3 \mathrm{~Hz}, 2 \mathrm{H}), 7.18-7.32(\mathrm{~m}, 5 \mathrm{H}), 7.40-7.65(\mathrm{~m}, 6 \mathrm{H}), 7.81(\mathrm{~s}, 1 \mathrm{H})$, $7.94(\mathrm{~s}, 1 \mathrm{H}), 8.39(\mathrm{~d}, J=7.5 \mathrm{~Hz}, 1 \mathrm{H}), 8.41$ (d, $J=7.7 \mathrm{~Hz}, 1 \mathrm{H}), 13.85$ (br s, $1 \mathrm{H}), 14.11$ (br s, $1 \mathrm{H}) ;{ }^{13} \mathrm{C}$ NMR $\left(100 \mathrm{MHz}, \mathrm{CDCl}_{3}\right) \delta$ 40.5, 58.3, 68.2, 109.8, 110.0, 116.0, 116.6, 124.4, 124.8, 125.20, 125.24, $127.0,127.30,127.32,127.35,127.46,128.3,128.8,129.4,129.58,129.9,136.60,136.63,137.0$, 163.7, 163.8, 168.5, 172.2; HRMS (FAB, 3-NOBA) calcd for $\mathrm{C}_{31} \mathrm{H}_{27} \mathrm{~N}_{2} \mathrm{O}_{2}(\mathrm{M}+\mathrm{H})^{+} 459.2073$, obsd 459.2086.

$N, N^{\prime}$-Bis(3',5'-diiodosalicylidene)-(1S)-benzyl-1,2-ethylenediamine (3e). Salen 3e was synthesized from (1S)-benzyl-1,2-ethylenediamine $(214 \mathrm{mg}, 1.437 \mathrm{mmol})$ and 3,5-diiodosalicylaldehyde (960 mg, 2.57 mmol, 1.8 equiv.), following General Procedure A, with heating for $24 \mathrm{~h}$ at $45^{\circ} \mathrm{C}$. Filtration and washing with cold ethanol provided pure 3e $(840 \mathrm{mg}, 76 \%):[\alpha]^{19}{ }_{\mathrm{D}}+43.9\left(c 1.41, \mathrm{CH}_{2} \mathrm{Cl}_{2}\right) ;{ }^{1} \mathrm{H}$ NMR $\left(400 \mathrm{MHz}, \mathrm{CDCl}_{3}\right) \delta 2.93(\mathrm{dd}, J=13.6,8.1 \mathrm{~Hz}, 1 \mathrm{H}), 3.10(\mathrm{dd}, J=13.6,4.4 \mathrm{~Hz}, 1 \mathrm{H}), 3.71-3.80(\mathrm{~m}$, 2H), 4.03 (br d, $J=10.2 \mathrm{~Hz}, 1 \mathrm{H}), 7.11$ (br d, $J=7.0 \mathrm{~Hz}, 1 \mathrm{H}), 7.19-7.30$ (m, 4H), 7.45 (d, $J=2.0 \mathrm{~Hz}$, $1 \mathrm{H}), 7.72(\mathrm{~s}, 1 \mathrm{H}), 7.99(\mathrm{~d}, J=2.0 \mathrm{~Hz}, 1 \mathrm{H}), 8.01(\mathrm{~d}, J=2.0 \mathrm{~Hz}, 1 \mathrm{H}), 8.08(\mathrm{~s}, 1 \mathrm{H}), 14.31$ (br s, 1H), 14.36 (br s, $1 \mathrm{H}) ;{ }^{13} \mathrm{C}$ NMR $\left(100 \mathrm{MHz}, \mathrm{CDCl}_{3}\right) \delta 40.7,63.1,70.9,79.4,79.6,87.3,87.5,119.5,119.6$, 127.0, 128.7, 129.4, 136.7, 139.9, 140.0, 148.8, 148.9, 160.3, 160.6, 163.8, 164.9; HRMS (FAB, 3NOBA) calcd for $\mathrm{C}_{23} \mathrm{H}_{19} \mathrm{O}_{2} \mathrm{~N}_{2} \mathrm{I}_{4}(\mathrm{M}+\mathrm{H})^{+}$862.7625, obsd 862.7643.

$\boldsymbol{N}, \boldsymbol{N}^{\prime}$-Bis(4'-benzyloxysalicylidene)-(1S)-benzyl-1,2-ethylenediamine (3f). From (1S)-benzyl-1,2ethylenediamine $(256 \mathrm{mg}, 1.71 \mathrm{mmol})$ and 4-benzyloxy-2-hydroxybenzaldehyde $(701 \mathrm{mg}, 3.07 \mathrm{mmol}$, 1.8 equiv.), following General Procedure A, with heating at $45^{\circ} \mathrm{C}$ for $24 \mathrm{~h}$, was obtained $3 \mathbf{f}$. Purification was achieved by trituration (cold ethanol) to give clean salen $(867 \mathrm{mg}, 99 \%):[\alpha]^{19}{ }_{\mathrm{D}}+99.5$ (c $\left.0.84, \mathrm{CH}_{2} \mathrm{Cl}_{2}\right)$; ${ }^{1} \mathrm{H}$ NMR (400 MHz, $\left.\mathrm{CDCl}_{3}\right) \delta 2.96(\mathrm{dd}, J=13.5,7.8 \mathrm{~Hz}, 1 \mathrm{H}), 3.09$ (dd, $J=13.5,4.4$ $\mathrm{Hz}, 1 \mathrm{H}), 3.62-3.68(\mathrm{~m}, 2 \mathrm{H}), 3.89(\mathrm{~d}, J=8.8 \mathrm{~Hz}, 1 \mathrm{H}), 5.05(\mathrm{~s}, 4 \mathrm{H}), 6.43(\mathrm{dd}, J=8.6,2.3 \mathrm{~Hz}, 1 \mathrm{H}), 6.46$ $(\mathrm{dd}, J=8.6,2.3 \mathrm{~Hz}, 1 \mathrm{H}), 6.52(\mathrm{dd}, J=3.8,2.4 \mathrm{~Hz}, 2 \mathrm{H}), 6.96(\mathrm{~d}, J=8.6 \mathrm{~Hz}, 1 \mathrm{H}), 7.08(\mathrm{~d}, J=8.6 \mathrm{~Hz}$, $1 \mathrm{H}), 6.96-7.44(\mathrm{~m}, 15 \mathrm{H}), 7.91(\mathrm{~s}, 1 \mathrm{H}), 8.15(\mathrm{~s}, 1 \mathrm{H}), 13.72(\mathrm{br} \mathrm{s}, 2 \mathrm{H}) ;{ }^{13} \mathrm{C} \mathrm{NMR}\left(100 \mathrm{MHz}, \mathrm{CDCl}_{3}\right) \delta$ $40.8,63.0,69.9,70.8,101.9,102.0,106.9,107.0,112.4,112.5,126.5,127.5,128.0,128.4,128.6$, $129.5,132.7,132.8,136.35,136.37,137.8,162.4,162.6,163.8,164.5,164.6,165.5$; HRMS (FAB, 3NOBA) calcd for $\mathrm{C}_{37} \mathrm{H}_{35} \mathrm{~N}_{2} \mathrm{O}_{4}(\mathrm{M}+\mathrm{H})^{+}$571.2597, obsd 571.2578. 
$N, N^{\prime}$-Bis(3',5'-di-tert-butylsalicylidene)-(1S)-( $\alpha$-naphthylmethyl)-1,2-ethylenediamine (4a). The requisite diamine, (1S)-( $\alpha$-naphthylmethyl)-1,2-ethylenediamine (4), was prepared as its dihydrochloride salt, from 3-( $\alpha$-naphthyl)-L-alanine (Chem-Impex International, 98.5\%). ${ }^{5}$ Following General Procedure A, from (1S)-( $\alpha$-naphthylmethyl)-1,2-ethylenediamine dihydrochloride (150 $\mathrm{mg}$, $0.549 \mathrm{mmol}), 3,5$-di-tert-butylsalicylaldehyde $(172 \mathrm{mg}, 1.1 \mathrm{mmol})$ and $\mathrm{NEt}_{3}(153 \mu \mathrm{L}, 1.1 \mathrm{mmol})$, after heating at $70{ }^{\circ} \mathrm{C}$ for $1 \mathrm{~h}$, followed by stirring another $5 \mathrm{~h}$ at $\mathrm{rt}$, was obtained $4 \mathbf{a}$. Filtration and trituration with ice-cold ethanol provided pure salen (329 mg, 95\%): $[\alpha]^{19} \mathrm{D}-36.4\left(c 1.26, \mathrm{CH}_{2} \mathrm{Cl}_{2}\right)$; ${ }^{1} \mathrm{H}$ NMR $\left(500 \mathrm{MHz}, \mathrm{CDCl}_{3}\right) \delta 1.21(\mathrm{~s}, 9 \mathrm{H}), 1.26(\mathrm{~s}, 9 \mathrm{H}), 1.41(\mathrm{~s}, 9 \mathrm{H}), 1.44(\mathrm{~s}, 9 \mathrm{H}), 3.34(\mathrm{dd}, J=13.9$, 7.7Hz, 1H), 3.65 (dd, $J=13.9,5.2 \mathrm{~Hz}, 1 \mathrm{H}), 3.82(\mathrm{dd}, J=11.7,4.2 \mathrm{~Hz}, 1 \mathrm{H}), 3.90(\mathrm{~m}, 1 \mathrm{H}), 4.0(\mathrm{dd}, J$ $=12,4.2 \mathrm{~Hz}, 1 \mathrm{H}), 6.83(\mathrm{~d}, J=2.4 \mathrm{~Hz}, 1 \mathrm{H}), 7.04(\mathrm{~d}, J=2.4 \mathrm{~Hz}, 1 \mathrm{H}), 7.33(\mathrm{~m}, 4 \mathrm{H}), 7.47(\mathrm{dt}, J=6.8,1.1$ $\mathrm{Hz}, 1 \mathrm{H}), 7.52(\mathrm{dt}, J=6.8,1.5 \mathrm{~Hz}, 1 \mathrm{H}), 7.72(\mathrm{~d}, J=7.9 \mathrm{~Hz}, 1 \mathrm{H}), 7.85(\mathrm{~d}, J=7.7 \mathrm{~Hz}, 1 \mathrm{H}), 7.96(\mathrm{~s}, 1 \mathrm{H})$, $8.06(\mathrm{~d}, J=8.3 \mathrm{~Hz}, 1 \mathrm{H}), 8.36(\mathrm{~s}, 1 \mathrm{H}), 13.6$ (br s, 1H), 13.7 (br s, 1H); ${ }^{13} \mathrm{C} \mathrm{NMR}\left(100 \mathrm{MHz}, \mathrm{CDCl}_{3}\right)$

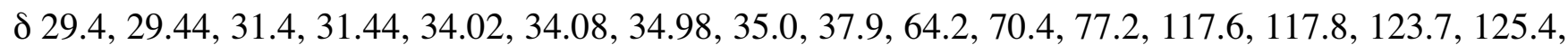
125.6, 126.0, 126.1, 126.1, 127.0, 127.1, 127.4, 128.2, 128.8, 131.9, 133.9, 134.1, 136.4, 136.6, 139.9, 140.0, 157.9, 158.1, 166.6, 167.8; HRMS (FAB, 3-NOBA) calcd for $\mathrm{C}_{43} \mathrm{H}_{57} \mathrm{~N}_{2} \mathrm{O}_{2}(\mathrm{M}+\mathrm{H})^{+} 633.442$, obsd 633.4440 .

$N, N^{\prime}$-Bis(3'-tert-butylsalicylidene)-(1S)-( $\alpha$-naphthylmethyl)-1,2-ethylenediamine (4b). Salen 4b was obtained from $(1 S)$-( $\alpha$-naphthylmethyl)-1,2-ethylenediamine (145 mg, $0.728 \mathrm{mmol})$ and 3-tertbutylsalicylaldehyde (260 mg, $1.46 \mathrm{mmol}$ ), following General Procedure A, heating for $2 \mathrm{~h}$ at $70{ }^{\circ} \mathrm{C}$, followed by stirring for $6 \mathrm{~h}$ at rt. Washing with ice-cold ethanol afforded the pure salen (309 $\mathrm{mg}, 82 \%)$ : $\left[\alpha{ }^{19}{ }_{\mathrm{D}}-24.1\left(c 1.31, \mathrm{CH}_{2} \mathrm{Cl}_{2}\right) ;{ }^{1} \mathrm{H}\right.$ NMR $\left(500 \mathrm{MHz}, \mathrm{CDCl}_{3}\right) \delta 1.43(\mathrm{~s}, 9 \mathrm{H}) ; 1.45(\mathrm{~s}, 9 \mathrm{H}), 3.34(\mathrm{dd}, J=$ $13.9,7.9 \mathrm{~Hz}, 1 \mathrm{H}), 3.69(\mathrm{dd}, J=13.8,4.8 \mathrm{~Hz}, 1 \mathrm{H}), 3.86(\mathrm{dd}, J=11.5,7.1 \mathrm{~Hz}, 1 \mathrm{H}) ; 3.90(\mathrm{~m}, 1 \mathrm{H}), 4.03$ (dd $J=11.6,4.4 \mathrm{~Hz}, 1 \mathrm{H}), 6.69(\mathrm{t}, J=7.6 \mathrm{~Hz}, 1 \mathrm{H}), 6.78(\mathrm{t}, J=7.6 \mathrm{~Hz}, 1 \mathrm{H}), 6.83(\mathrm{~d}, J=7.5 \mathrm{~Hz}, 1 \mathrm{H})$, $7.08(\mathrm{~d}, J=7.6 \mathrm{~Hz}, 1 \mathrm{H}), 7.3(\mathrm{~m}, 4 \mathrm{H}), 7.47(\mathrm{t}, J=7 \mathrm{~Hz}, 1 \mathrm{H}), 7.52(\mathrm{t}, J=8.2 \mathrm{~Hz}, 1 \mathrm{H}), 7.72(\mathrm{~d}, J=8 \mathrm{~Hz}$, $1 \mathrm{H}), 7.85$ (d, $J=8 \mathrm{~Hz}, 1 \mathrm{H}), 7.89$ (s, 1H), 8.07 (d, $J=8.3 \mathrm{~Hz}, 1 \mathrm{H}), 8.38$ (s, 1H), 13.8 (s, 1H); 13.9 (s, $1 \mathrm{H}) ;{ }^{13} \mathrm{C} \mathrm{NMR}\left(100 \mathrm{MHz}, \mathrm{CDCl}_{3}\right) \delta 29.3,29.3,34.8,34.81,37.8,64.2,70.4,117.7,117.8,118.3$, $118.5,123.7,125.3,125.6,126.2$, 127.5, 128.2, 128.9, 129.4, 129.6, 129.81, 129.84, 131.9, 133. 95, 133.97, 137.15, 137.4, 160.3, 160.4, 166.2, 167.5; HRMS (FAB, 3-NOBA) calcd for $\mathrm{C}_{35} \mathrm{H}_{41} \mathrm{~N}_{2} \mathrm{O}_{2}$ $(\mathrm{M}+\mathrm{H})^{+}$521.3168, obsd. 521.3157.

$N, N^{\prime}$-Bis(2'-hydroxy-1'-naphthylidene)-(1S)-( $\alpha$-naphthylmethyl)-1,2-ethylenediamine (4c). From (1S)-( $\alpha$-naphthylmethyl)-1,2-ethylenediamine dihydrochloride (150 mg, $0.549 \mathrm{mmol})$, 2-hydroxy-1naphthaldehyde $(189 \mathrm{mg}, 1.1 \mathrm{mmol})$ and $\mathrm{NEt}_{3}(1.1 \mathrm{mmol})$, following General Procedure A, with heating for $1 \mathrm{~h}$ at $70{ }^{\circ} \mathrm{C}$ and stirring for another $6 \mathrm{~h}$ at rt, was obtained $4 \mathrm{c}$. Filtration and crystallization from hot EtOH provided clean 4c (103 mg, 37\%): $[\alpha]^{19}{ }_{\mathrm{D}}^{-75.4}\left(\mathrm{c} 1.00, \mathrm{CH}_{2} \mathrm{Cl}_{2}\right)$; ${ }^{1} \mathrm{H} \mathrm{NMR}(500 \mathrm{MHz}$, $\left.\mathrm{CDCl}_{3}\right) \delta 3.44(\mathrm{dd}, J=13.8,7.8 \mathrm{~Hz}, 1 \mathrm{H}), 3.72(\mathrm{dd}, J=14,5.1 \mathrm{~Hz}, 1 \mathrm{H}), 3.89(\mathrm{~m}, 1 \mathrm{H}), 4.1(\mathrm{~m}, 2 \mathrm{H}), 6.92$ $(\mathrm{d}, J=9.2 \mathrm{~Hz}, 1 \mathrm{H}), 6.98(\mathrm{~d}, J=9.1 \mathrm{~Hz}, 1 \mathrm{H}), 7.15(\mathrm{t}, J=9.6 \mathrm{~Hz}, 1 \mathrm{H}), 7.17(\mathrm{t}, J=8.7 \mathrm{~Hz}, 1 \mathrm{H}), 7.25(\mathrm{~m}$, 2H), $7.37(\mathrm{~m}, 3 \mathrm{H}), 7.48(\mathrm{~m}, 2 \mathrm{H}), 7.54(\mathrm{~m}, 3 \mathrm{H}), 7.62(\mathrm{~m}, 3 \mathrm{H}), 7.73(\mathrm{~d}, J=7.3 \mathrm{~Hz}, 1 \mathrm{H}), 7.81(\mathrm{~d}, J=8.4$ 
$\mathrm{Hz}, 1 \mathrm{H}), 7.87(\mathrm{~d}, J=8 \mathrm{~Hz}, 1 \mathrm{H}), 8.06(\mathrm{~d}, J=8.4 \mathrm{~Hz}, 1 \mathrm{H}), 8.5(\mathrm{~s}, 1 \mathrm{H}), 8.8(\mathrm{~s}, 1 \mathrm{H}), 14.7$ (br s, $1 \mathrm{H}) ; 14.9$ (br s, $1 \mathrm{H}) ;{ }^{13} \mathrm{C} \mathrm{NMR}\left(100 \mathrm{MHz}, \mathrm{CDCl}_{3}\right) \delta 37.4,59.5,67.6,107.3,107.4,118.3,118.5,121.6,122.8$, $123.19,125.37,125.7,126.4,126.49,126.7,127.5,127.7,127.8,128.3,128.7,128.9,129,131.5$, 132.7, 132.77, 133.1, 133.9, 135.7, 136.5, 160.4, 160.5, 168.9, 172.1; HRMS (FAB, 3-NOBA) calcd for $\mathrm{C}_{35} \mathrm{H}_{29} \mathrm{~N}_{2} \mathrm{O}_{2}(\mathrm{M}+\mathrm{H})^{+} 509.2229$, obsd 509.2244.

$N, N^{\prime}$-Bis(3',5'-diiodosalicylidene)-(1S)-( $\alpha$-naphthylmethyl)-1,2-ethylenediamine (4e). Following General Procedure A, from (1S)-( $\alpha$-naphthylmethyl)-1,2-ethylenediamine dihydrochloride (150 mg, $0.549 \mathrm{mmol}$ ), 3,5-diiodosalicylaldehyde (410 mg, $1.1 \mathrm{mmol}$ ) and $\mathrm{NEt}_{3}$ (153 $\mu \mathrm{L}, 1.1 \mathrm{mmol}$ ), heating for $30 \mathrm{~min}$ at $70{ }^{\circ} \mathrm{C}$, followed by $4 \mathrm{~h}$ stirring at rt, was obtained $4 \mathbf{e}$. Purification was achieved by trituration with cold ethanol to give the final product (415 mg, 83\%): $[\alpha]{ }^{19}{ }_{\mathrm{D}}-35.9\left(c 1.02, \mathrm{CH}_{2} \mathrm{Cl}_{2}\right) ;{ }^{1} \mathrm{H}$ NMR $\left(500 \mathrm{MHz}, \mathrm{CDCl}_{3}\right) \delta 3.29(\mathrm{dd}, J=14,8.5 \mathrm{~Hz}, 1 \mathrm{H}), 3.62(\mathrm{dd}, J=14,5 \mathrm{~Hz}, 1 \mathrm{H}), 3.85(\mathrm{dd}, J=12.3,8.1$ $\mathrm{Hz}, 1 \mathrm{H}), 3.98(\mathrm{~m}, 1 \mathrm{H}), 4.1(\mathrm{dd}, J=12.4,2.9 \mathrm{~Hz}, 1 \mathrm{H}), 7.18(\mathrm{~d}, J=2 \mathrm{~Hz}, 1 \mathrm{H}), 7.21(\mathrm{~d}, J=6.8 \mathrm{~Hz}, 1 \mathrm{H})$, $7.33(\mathrm{t}, J=7.1 \mathrm{~Hz}, 1 \mathrm{H}), 7.46(\mathrm{~d}, J=2 \mathrm{~Hz}, 1 \mathrm{H}), 7.52(\mathrm{~m}, 2 \mathrm{H}), 7.74(\mathrm{~d}, J=8.2 \mathrm{~Hz}, 1 \mathrm{H}), 7.86(\mathrm{~d}, J=7.9$ $\mathrm{Hz}, 1 \mathrm{H}), 7.93$ (d, $J=8.3 \mathrm{~Hz}, 1 \mathrm{H}), 7.98(\mathrm{~d}, J=2 \mathrm{~Hz}, 1 \mathrm{H}), 8.01$ (d, $J=2 \mathrm{~Hz}, 1 \mathrm{H}), 8.11$ (s, 1H), 14.3 (s, 1H), $14.4(\mathrm{~s}, 1 \mathrm{H}) ;{ }^{13} \mathrm{C} \mathrm{NMR}\left(125 \mathrm{MHz}, \mathrm{CDCl}_{3}\right) \delta$ 37.8, 63.3, 69.7, 79.4, 79.5, 87.2, 87.5, 119.4, 119.6, $123.1,125.4,125.9,126.5,128.0,128.2,129.1,131.6,132.6,134,139.9,140.0,148.7,148.9,160.3$, 160.6, 163.8, 164.9; HRMS (FAB, 3-NOBA) calcd for $\mathrm{C}_{27} \mathrm{H}_{21} \mathrm{~N}_{2} \mathrm{O}_{2} \mathrm{I}_{4}(\mathrm{M}+\mathrm{H})^{+} 912.7782$, obsd 912.7801.

$N, N^{\prime}$-Bis(4'-benzyloxysalicylidene)-(1S)-( $\alpha$-naphthylmethyl)-1,2-ethylenediamine (4f). The title salen was accessed from $(1 S)$-( $\alpha$-naphthylmethyl)-1,2-ethylenediamine (110 mg, $0.549 \mathrm{mmol})$ and 4benzyloxysalicylaldehyde $(251 \mathrm{mg}, 1.10 \mathrm{mmol})$, following General Procedure A, heating for $2 \mathrm{~h}$ at 70 ${ }^{\circ} \mathrm{C}$, followed by stirring for $6 \mathrm{~h}$ at rt. Filtration and trituration with cold ethanol gave clean $4 \mathbf{f}(272 \mathrm{mg}$,

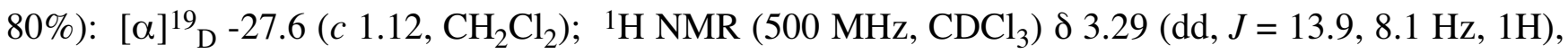
$3.62(\mathrm{dd}, J=13.9,5 \mathrm{~Hz}, 1 \mathrm{H}), 3.76(\mathrm{dd}, J=12,7.2 \mathrm{~Hz}, 1 \mathrm{H}), 3.83(\mathrm{~m}, 1 \mathrm{H}), 3.96(\mathrm{dd}, J=12,3.5 \mathrm{~Hz}, 1 \mathrm{H})$, $5.03(\mathrm{~s}, 2 \mathrm{H}), 5.04(\mathrm{~s}, 2 \mathrm{H}), 6.35(\mathrm{~d}, J=2.4 \mathrm{~Hz}, 1 \mathrm{H}), 6.37(\mathrm{~d}, J=2.4 \mathrm{~Hz}, 1 \mathrm{H}), 6.43(\mathrm{~d}, J=2.4 \mathrm{~Hz}, 1 \mathrm{H})$, $6.45(\mathrm{~d}, J=2.4 \mathrm{~Hz}, 1 \mathrm{H}), 6.49(\mathrm{~d}, J=2.3 \mathrm{~Hz}, 1 \mathrm{H}), 6.84(\mathrm{~d}, J=8.6 \mathrm{~Hz}, 1 \mathrm{H}), 7.07(\mathrm{~d}, J=8.6 \mathrm{~Hz}, 1 \mathrm{H})$, $7.26(\mathrm{~d}, J=7.2 \mathrm{~Hz}, 1 \mathrm{H}), 7.35(\mathrm{~m}, 11 \mathrm{H}), 7.47(\mathrm{t}, J=6.9 \mathrm{~Hz}, 1 \mathrm{H}), 7.53(\mathrm{dt}, J=8.4,1.3 \mathrm{~Hz}, 1 \mathrm{H}), 7.71(\mathrm{~d}$, $J=7.4 \mathrm{~Hz}, 1 \mathrm{H}), 7.72(\mathrm{~s}, 1 \mathrm{H}), 7.85(\mathrm{~d}, J=7.7 \mathrm{~Hz}, 1 \mathrm{H}), 8.01(\mathrm{~d}, J=8.3 \mathrm{~Hz}, 1 \mathrm{H}), 8.2(\mathrm{~s}, 1 \mathrm{H}), 13.7$ (br s, 2H); ${ }^{13} \mathrm{C} \mathrm{NMR}\left(100 \mathrm{MHz}, \mathrm{CDCl}_{3}\right) \delta 37.8,63.5,69.7,69.9,101.9,102.0,106.8,107.0,112.3,112.5$, $123.5,125.4,125.6,126.2,127.4,127.5,128.1,128.2$, 128.6, 128.9, 131.8, 132.7, 132.75, 133.8, 133.9, 136.36, 136.38, 162.4, 162.6, 163.8, 164.49, 164.52, 165.7; HRMS (FAB, 3-NOBA) calcd for $\mathrm{C}_{41} \mathrm{H}_{37} \mathrm{~N}_{2} \mathrm{O}_{4}(\mathrm{M}+\mathrm{H})^{+} 621.2753$, obsd. 621.2733 . 
$N, N^{\prime}$-Bis(8'-hydroxy-1',1',7',7'-tetramethyljulolidinyl-9'-methylidene)-(1S)- $\alpha$-naphthylmethyl-1,2ethylenediamine (4g). From (1S)-( $\alpha$-naphthylmethyl)-1,2-ethylenediamine (150 mg, $0.549 \mathrm{mmol}), 8$ hydroxy-1,1,7,7-tetramethyljulolidinyl-9-carboxaldehyde (300 mg, $1.1 \mathrm{mmol})$ and $\mathrm{NEt}_{3}(2.2 \mathrm{mmol})$, following General Procedure A, heating for $1 \mathrm{~h}$ at $70{ }^{\circ} \mathrm{C}$, followed by stirring for $5 \mathrm{~h}$ at $\mathrm{rt}$, was obtained a modest precipitate. Trituration with cold ethanol yielded clean salen $4 \mathbf{g}(117 \mathrm{mg}, 30 \%)$ : $[\alpha]^{19}{ }_{\mathrm{D}}-74.9$ (c $\left.0.71, \mathrm{CH}_{2} \mathrm{Cl}_{2}\right) ;{ }^{1} \mathrm{H} \mathrm{NMR}\left(400 \mathrm{MHz}, \mathrm{CDCl}_{3}\right) \delta 1.12(\mathrm{~s}, 3 \mathrm{H}), 1.15(\mathrm{~s}, 3 \mathrm{H}), 1.20(\mathrm{~s}, 3 \mathrm{H}), 1.22(\mathrm{~s}, 3 \mathrm{H})$, $1.45(\mathrm{~s}, 3 \mathrm{H}), 1.47(\mathrm{~s}, 3 \mathrm{H}), 1.49(\mathrm{~s}, 3 \mathrm{H}), 1.5(\mathrm{~s}, 3 \mathrm{H}), 1.70(\mathrm{~m}, 8 \mathrm{H}), 3.15(\mathrm{~m}, 10 \mathrm{H}), 3.70(\mathrm{~m}, 2 \mathrm{H}), 3.82$ $(\mathrm{dd}, J=11.3,4.7 \mathrm{~Hz}, 1 \mathrm{H}), 6.6(\mathrm{~s}, 3 \mathrm{H}), 6.8(\mathrm{~s}, 3 \mathrm{H}), 7.29(\mathrm{t}, J=6.1 \mathrm{~Hz}, 1 \mathrm{H}), 7.33(\mathrm{t}, J=7.1 \mathrm{~Hz}, 1 \mathrm{H})$, $7.45(\mathrm{t}, J=6.7 \mathrm{~Hz}, 1 \mathrm{H}), 7.49(\mathrm{t}, J=8.3 \mathrm{~Hz}, 1 \mathrm{H}), 7.67(\mathrm{~s}, 1 \mathrm{H}), 7.68(\mathrm{~d}, J=8.7 \mathrm{~Hz}, 1 \mathrm{H}), 7.82(\mathrm{~d}, J=7.8$ $\mathrm{Hz}, 1 \mathrm{H}), 8.07$ (d, J = 5 Hz, 1H), 8.07 (s, 1H), 14 (br s, 1H), 14.1 (br s, 1H); ${ }^{13} \mathrm{C} \mathrm{NMR}(100 \mathrm{MHz}$, $\left.\mathrm{CDCl}_{3}\right) \delta 28.46,28.53,31.1,31.14,31.6,31.7,32.19,32.2,36.6,38,40.1,46.9,47.3,63.8,70.0,108.5$, 108.7, 114.6, 114.8, 121.43, 121.6, 124.0, 125.4, 125.9, 126.9, 127.5, 127.6, 128.1, 128.6, 131.9, 133.8, 134.8, 145.4, 145.7, 160.3, 161.0, 164.8, 165.8; HRMS (FAB, 3-NOBA) calcd for $\mathrm{C}_{47} \mathrm{H}_{59} \mathrm{~N}_{4} \mathrm{O}_{2}$ $(\mathrm{M}+\mathrm{H})^{+} 711.4638$, obsd 711.4619 .

$N, N^{\prime}$-Bis(3',5'-di-tert-butylsalicylidene)-(1S)-( $\beta$-naphthylmethyl)-1,2-ethylenediamine (5a). The requisite diamine, (1S)-(2-naphthylmethyl)-1,2-ethylenediamine (5), was prepared from 3-( $\beta$-naphthyl)L-alanine (Chem-Impex International, 98.5\%,). ${ }^{5}$ From $(1 S)$-( $\beta$-naphthylmethyl)-1,2-ethylenediamine (145 mg, $0.728 \mathrm{mmol}$ ) and 3,5-di-tert-butylsalicylaldehyde (343 $\mathrm{mg}, 1.46 \mathrm{mmol})$, following General Procedure A, with stirring overnight at $50{ }^{\circ} \mathrm{C}$ was obtained 5a. Filtration, followed by washing with ice-cold ethanol, provided the pure product $(320 \mathrm{mg}, 85 \%):[\alpha]{ }^{19}{ }_{\mathrm{D}}+5.69\left(c 1.23, \mathrm{CH}_{2} \mathrm{Cl}_{2}\right) ;{ }^{1} \mathrm{H}$ NMR $\left(500 \mathrm{MHz}, \mathrm{CDCl}_{3}\right) \delta 1.22(\mathrm{~s}, 3 \mathrm{H}), 1.27$ (s, 3H), 1.43 (s, 3H), 1.45 (s, 3H), 3.19 (dd, J = 13.5, 7.6 Hz, 1H), 3.27 (dd, $J=13.5,5.4 \mathrm{~Hz}, 1 \mathrm{H}), 3.73(\mathrm{dd}, J=11.8,7.7 \mathrm{~Hz}, 1 \mathrm{H}), 3.86(\mathrm{~m}, 1 \mathrm{H}), 3.97(\mathrm{dd}, J=11.9$, $3.6 \mathrm{~Hz}, 1 \mathrm{H}), 6.9$ (br s, 1H), 7.04 (br d, $J=1.7 \mathrm{~Hz}, 1 \mathrm{H}), 7.35$ (m, 3H), 7.43 (m, 2H), 7.67 (br s, 1H), 7.77 (m, 3H), 8.15 (br s, 1H), 8.33 (br s, 1H), 13.6 (br s, 2H); ${ }^{13} \mathrm{C} \mathrm{NMR} \mathrm{(100} \mathrm{MHz,} \mathrm{CDCl}_{3}$ ) $\delta 29.38,29.42$, 31.38, 31.44, 34.02, 34.07, 35.0, 41.1, 63.8, 71.3, 77.2, 117.6, 117.8, 125.5, 125.97, 126.0, 126.16, $127.0,127.6,127.9,128.1,128.2,132.2,133.5,135.6,136.4,136.6,139.9,140.0,157.97,158.05$, 166.6, 167.7; HRMS (FAB, 3-NOBA) calcd for $\mathrm{C}_{43} \mathrm{H}_{57} \mathrm{~N}_{2} \mathrm{O}_{2}(\mathrm{M}+\mathrm{H})^{+} 633.4420$, obsd 633.4413.

$N, N^{\prime}$-Bis(3'-tert-butylsalicylidene)-(1S)-( $\beta$-naphthylmethyl)-1,2-ethylenediamine (5b). The title salen was obtained by following General Procedure A, from (1S)-( $\beta$-naphthylmethyl)-1,2ethylenediamine (145 mg, $0.728 \mathrm{mmol}$ ) and 3-tert-butylsalicylaldehyde (260 mg, $1.46 \mathrm{mmol})$, after heating overnight at $50{ }^{\circ} \mathrm{C}$. Precipitation was induced by the addition of a small amount of water. Filtration and trituration with ethanol yielded pure 5b $(399 \mathrm{mg}, 87 \%)$ : $[\alpha]^{19}{ }_{\mathrm{D}}+11.0\left(c 1.98, \mathrm{CH}_{2} \mathrm{Cl}_{2}\right)$; ${ }^{1} \mathrm{H}$ NMR $\left(500 \mathrm{MHz}, \mathrm{CDCl}_{3}\right) \delta 1.41(\mathrm{~s}, 3 \mathrm{H}), 1.44(\mathrm{~s}, 3 \mathrm{H}), 3.19(\mathrm{dd}, J=13.6,7.8 \mathrm{~Hz}, 1 \mathrm{H}), 3.28(\mathrm{dd}, J=$ $13.6,5.5 \mathrm{~Hz}, 1 \mathrm{H}), 3.76(\mathrm{dd}, J=12.1,7.6 \mathrm{~Hz}, 1 \mathrm{H}), 3.86(\mathrm{~m} .1 \mathrm{H}), 3.98(\mathrm{dd}, J=12.1,3.6 \mathrm{~Hz}, 1 \mathrm{H}), 6.7$ (t, $J=7.6 \mathrm{~Hz}, 1 \mathrm{H}), 6.77(\mathrm{t}, J=7.6 \mathrm{~Hz}, 1 \mathrm{H}), 6.91(\mathrm{~d}, J=6.7 \mathrm{~Hz}, 1 \mathrm{H}), 7.05(\mathrm{~d}, J=6.7 \mathrm{~Hz}, 1 \mathrm{H}), 7.27(\mathrm{dd}, J$ $=7.7,1.5 \mathrm{~Hz}, 1 \mathrm{H}), 7.29(\mathrm{dd}, J=7.7,1.5 \mathrm{~Hz}, 1 \mathrm{H}), 7.33(\mathrm{dd}, J=8.3,1.6 \mathrm{~Hz}, 1 \mathrm{H}), 7.42(\mathrm{~m}, 2 \mathrm{H}), 7.67(\mathrm{~s}$, $1 \mathrm{H}), 7.76(\mathrm{~m}, 3 \mathrm{H}), 8.12(\mathrm{~s}, 1 \mathrm{H}), 8.32(\mathrm{~s}, 1 \mathrm{H}), 13.8$ (br s, $2 \mathrm{H}) ;{ }^{13} \mathrm{C} \mathrm{NMR}\left(125 \mathrm{MHz}, \mathrm{CDCl}_{3}\right) \delta 29.3$, 
$29.35,34.8,41.1,63.8,71.2,117.76,117.83,118.4,118.5,125.5,126.0,127.56,127.6,127.86,128.1$, $128.2,129.5,129.52,129.8,129.9,132.3,133.5,135.4,137.2,137.4,160.3,160.4,166.2,167.4$; HRMS (FAB, 3-NOBA) calcd for $\mathrm{C}_{35} \mathrm{H}_{41} \mathrm{~N}_{2} \mathrm{O}_{2}(\mathrm{M}+\mathrm{H})^{+} 521.3168$, obsd 521.3150.

$N, N^{\prime}$-Bis(2'-hydroxy-1'-naphthylidene)-(1S)- $\beta$-naphthylmethyl)-1,2-ethylenediamine $\quad(5 \mathrm{c})$. Following General Procedure A, stirring overnight at $50{ }^{\circ} \mathrm{C}$, from $(1 S)$-( $\beta$-naphthylmethyl)-1,2ethylenediamine (145 mg, $0.728 \mathrm{mmol}$ ) and 2-hydroxy-1-naphthaldehyde (252 mg, $1.46 \mathrm{mmol}$ ), was obtained 5c. Purification by trituration with ethanol yielded a bright yellow solid (332 $\mathrm{mg}, 90 \%)$ : $[\alpha]^{19}{ }_{\mathrm{D}}-7.30\left(c 0.61, \mathrm{CH}_{2} \mathrm{Cl}_{2}\right) ;{ }^{1} \mathrm{H}$ NMR $\left(500 \mathrm{MHz}, \mathrm{DMSO}_{6}\right) \delta 3.23(\mathrm{dd}, J=13.8,8.5 \mathrm{~Hz}, 1 \mathrm{H}), 3.34$ $(\mathrm{m}, 1 \mathrm{H}), 4.02(\mathrm{dd}, J=13.1,3.3 \mathrm{~Hz}, 1 \mathrm{H}), 4.41(\mathrm{~m}, 1 \mathrm{H}), 6.74(\mathrm{~d}, J=9.3 \mathrm{~Hz}, 1 \mathrm{H}), 6.81(\mathrm{~d}, J=9.2 \mathrm{~Hz}$, 1H), 7.18 (app q, $J=7.7 \mathrm{~Hz}, 2 \mathrm{H}), 7.32(\mathrm{t}, J=7.6 \mathrm{~Hz}, 1 \mathrm{H}), 7.39(\mathrm{t}, J=7.6 \mathrm{~Hz}, 1 \mathrm{H}), 7.48(\mathrm{~m}, 2 \mathrm{H}), 7.62$ $(\mathrm{d}, J=7.8 \mathrm{~Hz}, 2 \mathrm{H}), 7.71(\mathrm{~d}, J=9.3 \mathrm{~Hz}, 1 \mathrm{H}), 7.72(\mathrm{~d}, J=9.3 \mathrm{~Hz}, 1 \mathrm{H}), 7.84(\mathrm{~m}, 4 \mathrm{H}), 8.02(\mathrm{~d}, J=8.4 \mathrm{~Hz}$, 1H), 9.07 (s, 1H), 9.2 (s, 1H), 14.4 (br s, 1H), 14.6 (br s, 1H); ${ }^{13} \mathrm{C}$ NMR (100 MHz, DMSO-d 6 ) $\delta 56.1$, 65.0, 106.4, 106.6, 118.7, 118.8, 122.4, 122.5, 123.1, 124.2, 125.5, 125.8, 126.05, 127.3, 127.4, 127.6, 127.6, 127.7 127.8, 128.7, 131.8, 133.0, 133.3, 133.8, 135.0, 136.1, 136.7, 160.3, 160.7, 172.1, 174.8; HRMS (FAB, 3-NOBA) calcd for $\mathrm{C}_{35} \mathrm{H}_{29} \mathrm{~N}_{2} \mathrm{O}_{2}(\mathrm{M}+\mathrm{H})^{+} 509.2229$, obsd 509.2242.

$N, N^{\prime}$-Bis(1'-hydroxy-2'-naphthylidene)-(1S)-( $\beta$-naphthylmethyl)-1,2-ethylenediamine (5d). The target salen was obtained following General Procedure A, with stirring overnight at $50{ }^{\circ} \mathrm{C}$, starting from (1S)-( $\beta$-naphthylmethyl)-1,2-ethylenediamine (145 mg, $0.728 \mathrm{mmol})$ and 1-hydroxy-2-naphthaldehyde (252 $\mathrm{mg}, 1.45 \mathrm{mmol})$. Filtration and washing with cold ethanol provided pure $5 \mathbf{d}$ was (323 $\mathrm{mg}, 87 \%$ ): $[\alpha]^{19}{ }_{\mathrm{D}}+536\left(c 1.29, \mathrm{CH}_{2} \mathrm{Cl}_{2}\right) ;{ }^{1} \mathrm{H}$ NMR $\left(500 \mathrm{MHz}, \mathrm{CDCl}_{3}\right) \delta 3.22(\mathrm{dd}, J=13.8,7.9 \mathrm{~Hz}, 1 \mathrm{H}), 3.3(\mathrm{dd}$, $J=13.8,5.3 \mathrm{~Hz}, 1 \mathrm{H}), 3.75(\mathrm{dd}, J=12.8,7.7 \mathrm{~Hz}, 1 \mathrm{H}), 3.91(\mathrm{~m}, 1 \mathrm{H}), 3.97(\mathrm{dd}, J=12.8,3.6 \mathrm{~Hz}, 1 \mathrm{H})$, $6.84(\mathrm{t}, J=8.7 \mathrm{~Hz}, 2 \mathrm{H}), 6.91(\mathrm{dd}, J=8.35,1.3 \mathrm{~Hz}, 2 \mathrm{H}), 7.33(\mathrm{dd}, J=8.35,1.3 \mathrm{~Hz}, 1 \mathrm{H}), 7.44(\mathrm{~m}, 3 \mathrm{H})$, $7.53(\mathrm{~m}, 3 \mathrm{H}), 7.59$ (t, $J=8.3 \mathrm{~Hz}, 2 \mathrm{H}), 7.68(\mathrm{br} \mathrm{s}, 1 \mathrm{H}), 7.77(\mathrm{~m}, 3 \mathrm{H}), 7.85(\mathrm{br} \mathrm{s}, 1 \mathrm{H}), 7.94(\mathrm{br} \mathrm{s}, 1 \mathrm{H})$,

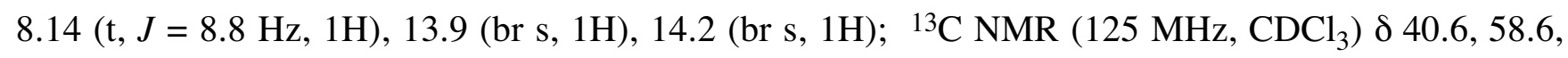
68.2, 109.8, 110.1, 116.03, 116.63, 124.4, 124.8, 125.2, 125.2, 125.8, 126.3, 127.29, 127.3, 127.35, 127.4, 127.46, 127.58, 127.6, 128.2, 128.3, 128.5, 129.4, 129.8, 132.4, 133.5, 134.2, 136.6, 137, 163.8, 163.9, 168.2, 171.8; HRMS (FAB, 3-NOBA) calcd for $\mathrm{C}_{35} \mathrm{H}_{29} \mathrm{~N}_{2} \mathrm{O}_{2}(\mathrm{M}+\mathrm{H})^{+}$509.2229, obsd 509.2210.

$N, N^{\prime}$-Bis(3',5'-diiodosalicylidene)-(1S)-( $\beta$-naphthylmethyl)-1,2-ethylenediamine (5e). Following General Procedure A, from (1S)-( $\beta$-naphthylmethyl)-1,2-ethylenediamine $(145 \mathrm{mg}, 0.728 \mathrm{mmol})$ and 3,5-diiodosalicylaldehyde (547 mg, $1.46 \mathrm{mmol}$ ), with stirring overnight at $50{ }^{\circ} \mathrm{C}$, was obtained $5 \mathbf{e}$. Filtration and further purification by trituration (cold ethanol) provided clean salen $(571 \mathrm{mg}, 85 \%)$ : $[\alpha]^{19}{ }_{\mathrm{D}}+7.52\left(c 1.05, \mathrm{CH}_{2} \mathrm{Cl}_{2}\right) ;{ }^{1} \mathrm{H} \mathrm{NMR}\left(400 \mathrm{MHz}, \mathrm{CDCl}_{3}\right) \delta 3.1(\mathrm{dd}, J=13.6,8.4 \mathrm{~Hz}, 1 \mathrm{H}), 3.26(\mathrm{dd}$, $J=13.6,5.1 \mathrm{~Hz}, 1 \mathrm{H}), 3.78(\mathrm{dd}, J=12.4,8 \mathrm{~Hz}, 1 \mathrm{H}), 3.89(\mathrm{~m}, 1 \mathrm{H}), 4.06(\mathrm{dd}, J=12.3,2.7 \mathrm{~Hz}, 1 \mathrm{H}), 7.23$ (d, $J=2 \mathrm{~Hz}, 1 \mathrm{H}), 7.25(\mathrm{~m}, 1 \mathrm{H}), 7.44(\mathrm{~m}, 3 \mathrm{H}), 7.58(\mathrm{br} \mathrm{s}, 1 \mathrm{H}), 7.76(\mathrm{~m}, 4 \mathrm{H}), 7.99(\mathrm{~d}, J=2 \mathrm{~Hz}, 1 \mathrm{H})$, $8.01(\mathrm{~d}, J=2 \mathrm{~Hz}, 1 \mathrm{H}), 8.07$ (br s, $1 \mathrm{H}), 14.4$ (br s, $2 \mathrm{H}) ;{ }^{13} \mathrm{C} \mathrm{NMR}\left(100 \mathrm{MHz}, \mathrm{CDCl}_{3}\right) \delta 40.9,63.1,70.8$, 77.2, 79.5, 79.6, 87.2, 87.5, 119.4, 119.6, 125.8, 126.3, 127.3, 127.5, 127.6, 128.1, 128.4, 132.3, 133.4, 
134.2, 134.2, 139.9, 139.95, 148.7, 148.8, 160.2, 160.5, 163.8, 164.9; HRMS (FAB, 3-NOBA) calcd for $\mathrm{C}_{27} \mathrm{H}_{21} \mathrm{~N}_{2} \mathrm{O}_{2} \mathrm{I}_{4}(\mathrm{M}+\mathrm{H})^{+}$912.7782, obsd 912.7769 .

$N, N^{\prime}$-Bis(4'-benzyloxysalicylidene)-(1S)-( $\beta$-naphthylmethyl)-1,2-ethylenediamine (5f): Salen $5 f$ was obtained by heating overnight at $50{ }^{\circ} \mathrm{C}$, following General Procedure A, starting from $(1 S)$ - $(\beta$ naphthylmethyl)-1,2-ethylenediamine (145 mg, $0.728 \mathrm{mmol}$ ) and 4-benzyloxysalicylaldehyde (334 $\mathrm{mg}$, $1.46 \mathrm{mmol})$. Trituration of the precipitated salen with ethanol provided pure salen (413 $\mathrm{mg}, 91 \%)$ : $[\alpha]^{19}{ }_{\mathrm{D}}+23.8\left(c 1.72, \mathrm{CH}_{2} \mathrm{Cl}_{2}\right) ;{ }^{1} \mathrm{H} \mathrm{NMR}\left(400 \mathrm{MHz}, \mathrm{CDCl}_{3}\right) \delta 3.11(\mathrm{dd}, J=14,8 \mathrm{~Hz}, 1 \mathrm{H}), 3.24(\mathrm{dd}, J$ = 14, $5.2 \mathrm{~Hz}, 1 \mathrm{H}), 3.67(\mathrm{dd}, J=12,7.4 \mathrm{~Hz}, 1 \mathrm{H}), 3.75(\mathrm{~m}, 1 \mathrm{H}), 3.92(\mathrm{dd}, J=12,3.2 \mathrm{~Hz}, 1 \mathrm{H}), 5.03(\mathrm{~s}$, $3 \mathrm{H}), 5.04(\mathrm{~s}, 3 \mathrm{H}), 6.38(\mathrm{dd}, J=8.6,2.4 \mathrm{~Hz}, 1 \mathrm{H}), 6.43(\mathrm{dd}, J=8.6,2.4 \mathrm{~Hz}, 1 \mathrm{H}), 6.50(\mathrm{~m}, 2 \mathrm{H}), 6.91(\mathrm{~d}, J$ $=8.6 \mathrm{~Hz}, 1 \mathrm{H}), 7.06(\mathrm{~d}, J=8.6 \mathrm{~Hz}, 1 \mathrm{H}), 7.4(\mathrm{~m}, 13 \mathrm{H}), 7.63(\mathrm{br} \mathrm{s}, 1 \mathrm{H}), 7.76(\mathrm{~m}, 3 \mathrm{H}), 7.92(\mathrm{br} \mathrm{s}, 1 \mathrm{H})$, 8.15 (br s, 1H), 13.7 (br s, 2H); ${ }^{13} \mathrm{C}$ NMR (125 MHz, $\left.\mathrm{CDCl}_{3}\right) \delta 41.0,63.2,69.9,70.9,102,102.1$, 106.9, 107.1, 112.4, 112.5, 125.5, 126.04, 127.48, 127.55, 127.6, 127.8, 128.07, 128.1, 128.6, 132.2, $132.7,132.8,133.5,135.4,136.44,136.43$ 162.5, 162.6, 163.8, 164.5, 164.6, 165.64; HRMS (FAB, 3NOBA) calcd for $\mathrm{C}_{41} \mathrm{H}_{36} \mathrm{~N}_{2} \mathrm{O}_{4}(\mathrm{M}+\mathrm{H})^{+} 621.2753$, obsd. 621.2772 .

\section{$N, N^{\prime}$-Bis $\left(8^{\prime}\right.$-hydroxy-1',1',7',7'-tetramethyljulolidinyl-9'-methylidene)-(1S)-( $\beta$-naphthylmethyl)-} 1,2-ethylenediamine (5g): Following General Procedure A, from (1S)-( $\beta$-naphthylmethyl)-1,2ethylenediamine (145 mg, $0.728 \mathrm{mmol}$ ) and 8-hydroxy-1,1,7,7-tetramethyljulolidinyl-9-carboxaldehyde (400 mg, $1.46 \mathrm{mmol}$ ), after heating overnight at $50{ }^{\circ} \mathrm{C}$, was obtained $\mathbf{5 g}$. Filtration and trituration with cold ethanol provided a pure sample $(444 \mathrm{mg}, 86 \%):[\alpha]{ }^{19} \mathrm{D}+143\left(c 1.54, \mathrm{CH}_{2} \mathrm{Cl}_{2}\right) ;{ }^{1} \mathrm{H}$ NMR (400 $\left.\mathrm{MHz}, \mathrm{CDCl}_{3}\right) \delta 1.15(\mathrm{~s}, 6 \mathrm{H}), 1.2(\mathrm{~s}, 3 \mathrm{H}), 1.22(\mathrm{~s}, 3 \mathrm{H}), 1.46(\mathrm{~s}, 3 \mathrm{H}), 1.48(\mathrm{~s}, 6 \mathrm{H}), 1.51(\mathrm{~s}, 3 \mathrm{H}), 1.70(\mathrm{~m}$, $8 \mathrm{H}), 3.15(\mathrm{~m}, 10 \mathrm{H}), 3.6(\mathrm{dd}, J=12,7.1 \mathrm{~Hz}, 1 \mathrm{H}), 3.67(\mathrm{~m}, 1 \mathrm{H}), 3.78(\mathrm{dd}, J=12,1 \mathrm{~Hz}, 1 \mathrm{H}), 6.67(\mathrm{~s}$, $3 \mathrm{H}), 6.8(\mathrm{~s}, 3 \mathrm{H}), 7.3(\mathrm{dd}, J=8.4,1.3 \mathrm{~Hz}, 1 \mathrm{H}), 7.41(\mathrm{~m}, 2 \mathrm{H}), 7.65(\mathrm{~s}, 1 \mathrm{H}), 7.75(\mathrm{~m}, J=8.9 \mathrm{~Hz}, 3 \mathrm{H}), 7.87$ (s, 1H), 8.02 (s, 1H), 14 (br s, 2H); ${ }^{13} \mathrm{C}$ NMR (100 MHz, $\mathrm{CDCl}_{3}$ ) $\delta$ 28.47, 28.53, 31.1, 31.2, 31.65, 31.7, 32.2, 36.6, 40.13, 41.13, 47.0, 47.35, 63.2, 71, 77.2, 108.6, 108.7, 114.7, 114.8, 121.5, 121.6, 125.2, 125.7, 127.5, 127.56, 127.61, 127.65, 127.8, 128.1, 128.2, 132.1, 133.5, 136.3, 145.5, 145.7, 160.3, 161.15, 164.8, 165.7; HRMS (FAB, 3-NOBA) calcd for $\mathrm{C}_{47} \mathrm{H}_{59} \mathrm{~N}_{4} \mathrm{O}_{2}(\mathrm{M}+\mathrm{H})^{+} 711.4638$, obsd 711.4669 .

\section{$N, N^{\prime}$-Bis(3',5'-di-tert-butylsalicylidene)-3- $O$-methyl-4,5-O-isopropylidene-1,2-dideoxy-D-}

fructopyranosyl-1,2-diamine (6a \& 7a): Diamines 6/7 were obtained as an anomeric mixture (favoring the $\beta$-anomer $\sim 9: 1$ in $\mathrm{CDCl}_{3}$ ) in three steps $\left[\mathrm{Tf}_{2} \mathrm{O}\right.$, pyridine; (ii) $\mathrm{NaN}_{3}$, DMF; (iii) $\mathrm{H}_{2}, \mathrm{PtO}_{2}$ ] from the known $\mathrm{D}$-fructose derivative, 2-azido-2-deoxy-3-O-methyl-4,5- $O$-isopropylidene- $\beta-D$ fructopyranose. ${ }^{6}$ General Procedure A was followed, with heating overnight at $50{ }^{\circ} \mathrm{C}$, from anomeric diamines 6/7 (162 mg, $0.700 \mathrm{mmol})$ and 3,5-di-tert-butylsalicylaldehyde (328 mg, $1.40 \mathrm{mmol}) . \mathrm{SiO}_{2}$ column chromatography $\left(0 \rightarrow 20 \% \mathrm{Et}_{2} \mathrm{O}\right.$-hexanes), provided both the $\beta$-anomer $7 \mathbf{a}$ ( $260 \mathrm{mg}, 56 \%$, elutes first) and the $\alpha$-anomer $6 \mathbf{6}(87 \mathrm{mg}, 19 \%)$. 
$\boldsymbol{\beta}$-Anomer (7a): $[\alpha]^{19}{ }_{\mathrm{D}}-120\left(c\right.$ 1.00, $\left.\mathrm{CH}_{2} \mathrm{Cl}_{2}\right)$; ${ }^{1} \mathrm{H}$ NMR $\left(600 \mathrm{MHz}, \mathrm{CDCl}_{3}\right) \delta 1.3(\mathrm{~s}, 9 \mathrm{H}), 1.31(\mathrm{~s}$, 9H), 1.34 (s, 3H), 1.43 (s, 9H), 1.44 (s, 9H), 1.57 (s, 3H), 3.68 (s, 3H), 3.75 (d, J=12 Hz, 1H), 3.8 (d, J $=6 \mathrm{~Hz}, 1 \mathrm{H}), 3.93(\mathrm{dd}, J=12,3.2 \mathrm{~Hz}, 1 \mathrm{H}), 3.96(\mathrm{dd}, J=12,2.3 \mathrm{~Hz}, 1 \mathrm{H}), 4.04(\mathrm{br} \mathrm{d}, J=12 \mathrm{~Hz}, 1 \mathrm{H})$, $4.16(\mathrm{~m}, 1 \mathrm{H}), 4.29(\operatorname{app~t}, J=6 \mathrm{~Hz}, 1 \mathrm{H}), 7.08(\mathrm{~d}, J=2 \mathrm{~Hz}, 1 \mathrm{H}), 7.16(\mathrm{~d}, J=2 \mathrm{~Hz}, 1 \mathrm{H}), 7.38(\mathrm{~d}, J=2$ $\mathrm{Hz}, 1 \mathrm{H}), 7.42$ (d, $J=2 \mathrm{~Hz}, 1 \mathrm{H}), 8.36(\mathrm{~s}, 1 \mathrm{H}), 8.61(\mathrm{~s}, 1 \mathrm{H}), 13.4(\mathrm{~s}, 1 \mathrm{H}), 13.6(\mathrm{~s}, 1 \mathrm{H}) ;{ }^{13} \mathrm{C}$ NMR $(150$

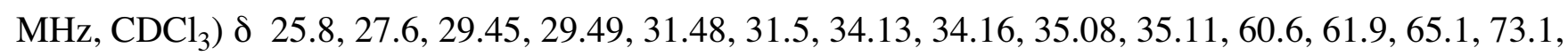
76.4, 79.9, 90.5, 109.3, 117.7, 118.0, 126.1, 127, 127.2, 127.8, 136.8, 137, 140.0, 140.3, 157.9, 158.3, 165.6, 169.0; HRMS (FAB, 3-NOBA) calcd for $\mathrm{C}_{40} \mathrm{H}_{60} \mathrm{O}_{6} \mathrm{~N}_{2}(\mathrm{M}+\mathrm{H})^{+} 665.4530$, obsd 665.4551.

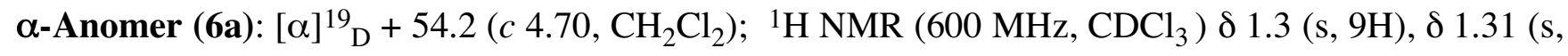
9H), $1.33(\mathrm{~s}, 3 \mathrm{H}), 1.43(\mathrm{~s}, 9 \mathrm{H}), 1.43(\mathrm{~s}, 9 \mathrm{H}), 1.44(\mathrm{~s}, 3 \mathrm{H}), 3.58(\mathrm{~d}, J=7 \mathrm{~Hz}, 1 \mathrm{H}), 3.72(\mathrm{~m}, 5 \mathrm{H}), 4.06$ $(\mathrm{d}, J=13 \mathrm{~Hz}, 1 \mathrm{H}), 4.12(\mathrm{dd}, J=12,5, \mathrm{~Hz}, 1 \mathrm{H}), 4.42(\mathrm{~m}, 2 \mathrm{H}), 7.07(\mathrm{~d}, J=2 \mathrm{~Hz}, 1 \mathrm{H}), 7.18(\mathrm{~d}, J=2$ $\mathrm{Hz}, 1 \mathrm{H}), 7.37$ (d, $J=2 \mathrm{~Hz}, 1 \mathrm{H}), 7.41$ (d, $J=2 \mathrm{~Hz}, 1 \mathrm{H}), 8.34(\mathrm{~s}, 1 \mathrm{H}), 8.63(\mathrm{~s}, 1 \mathrm{H}), 13.3(\mathrm{~s}, 1 \mathrm{H}), 13.6$

(br s, $1 \mathrm{H}) ;{ }^{13} \mathrm{C} \mathrm{NMR}\left(150 \mathrm{MHz}, \mathrm{CDCl}_{3}\right) \delta 24.6,26.9,29.4,29.5,31.5,34.1,34.13,35.04,35.07,61.0$, 62.6, 62.8, 71.8, 76.2, 84.5, 91.1, 109.6, 117.7, 117.9, 126.1, 127.2, 127.5,136.7, 136.8, 140.0, 140.3, 157.9, 158.3, 162.5, 169.0; HRMS (FAB, 3-NOBA) calcd for $\mathrm{C}_{40} \mathrm{H}_{60} \mathrm{O}_{6} \mathrm{~N}_{2}(\mathrm{M}+\mathrm{H})^{+} 665.4530$, obsd 665.4515.

\section{$N, N^{\prime}$-Bis(3'-tert-butylsalicylidene)-3- $O$-methyl-4,5- $O$-isopropylidene-1,2-dideoxy-D-}

fructopyranosyl-1,2-diamine $(6 \mathbf{b} \& \mathbf{7 b})$ : A mixure of diastereomeric salens was obtained from anomeric diamines $6 / 7$ (130 mg, $0.560 \mathrm{mmol})$ and 3-tert-butylsalicylaldehyde (200 $\mathrm{mg}, 1.12 \mathrm{mmol})$, by heating overnight at $50{ }^{\circ} \mathrm{C}$, following General Procedure A. Purification by silica gel column chromatography $\left(0 \rightarrow 20 \% \mathrm{Et}_{2} \mathrm{O}\right.$-hexanes), yielded both the $\beta$-anomer $\mathbf{7 b}$ (170 $\mathrm{mg}, 55 \%$, elutes first) and the $\alpha$-anomer $\mathbf{6 b}(26 \mathrm{mg}, 8 \%)$.

$\boldsymbol{\beta}$-Anomer (7b): $[\alpha]{ }^{19} \mathrm{D}-157$ (c 2.40, $\mathrm{CH}_{2} \mathrm{Cl}_{2}$ ); ${ }^{1} \mathrm{H} \mathrm{NMR}\left(600 \mathrm{MHz}, \mathrm{CDCl}_{3}\right) \delta 1.35$ (s, 3H), 1.43 (s, 9H), 1.45 (s, 9H), 1.58 (s, 3H), $3.68(\mathrm{~s}, 3 \mathrm{H}), 3.77$ (br d, $J=12 \mathrm{~Hz}, 1 \mathrm{H}), 3.82$ (d, $J=7 \mathrm{~Hz}, 1 \mathrm{H}), 3.92-3.96$ $(\mathrm{dd}, J=13,3 \mathrm{~Hz}, 1 \mathrm{H}), 3.96-4.01$ (dd, $J=13 \mathrm{~Hz}, 1 \mathrm{H}), 4.06(\mathrm{br} \mathrm{d}, J=12 \mathrm{~Hz}, 1 \mathrm{H}), 4.17-4.2(\mathrm{~m}, 1 \mathrm{H})$, 4.32(app t, $J=7 \mathrm{~Hz}, 1 \mathrm{H}), 6.81(\operatorname{app} \mathrm{t}, J=7 \mathrm{~Hz}, 1 \mathrm{H}), 6.84(\operatorname{app~t}, J=7 \mathrm{~Hz}, 1 \mathrm{H}), 7.13(\mathrm{~d}, J=8 \mathrm{~Hz}, 1 \mathrm{H})$, $7.20(\mathrm{~d}, J=8 \mathrm{~Hz}, 1 \mathrm{H}), 7.33(\mathrm{~d}, J=8 \mathrm{~Hz}, 1 \mathrm{H}), 7.37$ (d, $J=8 \mathrm{~Hz}, 1 \mathrm{H}), 8.37(\mathrm{~s}, 1 \mathrm{H}), 8.62(\mathrm{~s}, 1 \mathrm{H}), 13.6$ (s, 1H), $13.8(\mathrm{~s}, 1 \mathrm{H}) ;{ }^{13} \mathrm{C} \mathrm{NMR}\left(150 \mathrm{MHz}, \mathrm{CDCl}_{3}\right) \delta$ 25.8, 27.6, 29.3, 29.4, 34.8, 34.9, 60.6, 61.9, 64.9, 73.0, 76.2, 79.7, 90.5 109.5, 117.8, 117.9, 118.5, 118.8, 129.6, 129.9, 130.2, 130.9, 137.6, 137.7, 160.6, 160.7, 165.3, 168.6; HRMS (FAB, 3-NOBA) calcd for $\mathrm{C}_{32} \mathrm{H}_{44} \mathrm{O}_{6} \mathrm{~N}_{2}(\mathrm{M}+\mathrm{H})^{+}$553.3279, obsd 553.3277.

$\boldsymbol{\alpha}$-Anomer (6b): $[\alpha]^{19}{ }_{\mathrm{D}}+62.2\left(c 1.28, \mathrm{CH}_{2} \mathrm{Cl}_{2}\right){ }^{1} \mathrm{H} \mathrm{NMR}\left(600 \mathrm{MHz}, \mathrm{CDCl}_{3}\right) \delta 1.31(\mathrm{~s}, 3 \mathrm{H}), 1.41(\mathrm{~s}$, $9 \mathrm{H}), 1.43$ (s, 9H), $3.61(\mathrm{~d}, J=7 \mathrm{~Hz}, 1 \mathrm{H}), 3.68-3.71(\mathrm{~m}, 5 \mathrm{H}), 4.06$ (br d, $J=13 \mathrm{~Hz}, 1 \mathrm{H}), 4.10(\mathrm{dd}, J=$ $12,5 \mathrm{~Hz}, 1 \mathrm{H}), 4.38-4.43(\mathrm{~m}, 2 \mathrm{H}), 6.78(\operatorname{app~t}, J=8 \mathrm{~Hz}, 1 \mathrm{H}), 6.82(\operatorname{app~t}, J=8 \mathrm{~Hz}, 1 \mathrm{H}), 7.09$ (dd, $J=8$, $1.5 \mathrm{~Hz}, 1 \mathrm{H}), 7.18(\mathrm{dd}, J=8,1.5 \mathrm{~Hz}, 1 \mathrm{H}), 7.31(\mathrm{dd}, J=8,1.5 \mathrm{~Hz}, 1 \mathrm{H}), 7.34(\mathrm{dd}, J=8,1.5 \mathrm{~Hz}, 1 \mathrm{H}), 8.32$ 
(s, 1H), $8.61(\mathrm{~s}, 1 \mathrm{H}), 13.5(\mathrm{~s}, 1 \mathrm{H}), 13.9$ (br s, 1H); ${ }^{13} \mathrm{C} \mathrm{NMR}\left(150 \mathrm{MHz}, \mathrm{CDCl}_{3}\right) \delta 24.5,26.8,29.3$, 29.4, 34.8, 34.9, 60.9, 62.6, 62.9, 71.7, 75.8, 83.9, 91.1, 109.6, 117.8, 118.1, 118.4, 118.6, 129.6, 129.9, 130.8, 137.4, 137.6, 160.2, 160.6, 162.2, 168.7; HRMS (FAB, 3-NOBA) calcd for $\mathrm{C}_{32} \mathrm{H}_{44} \mathrm{O}_{6} \mathrm{~N}_{2}$ $(\mathrm{M}+\mathrm{H})^{+}$553.3279, obsd 553.3298.

\section{$N, N^{\prime}$-Bis(2'-hydroxy-1'-naphthylidene)-3-O-methyl-4,5-O-isopropylidene-1,2-dideoxy-D-}

fructopyranosyl-1,2-diamine (6c \& 7c). General Procedure A was followed, starting from anomeric diamines 6/7 (162 mg, $0.700 \mathrm{mmol})$ and 2-hydroxy-1-naphthaldehyde (241 mg, $1.40 \mathrm{mmol})$, with heating overnight at $50{ }^{\circ} \mathrm{C}$. Separation of the product mixture by $\mathrm{SiO}_{2}$ column chromatography $\left(0 \rightarrow 55 \% \mathrm{Et}_{2} \mathrm{O}\right.$-hexanes), provided both the $\beta$-anomer 7c (170 mg, 45\%, elutes first) and the $\alpha$-anomer 6c (15 mg, 4\%).

$\boldsymbol{\beta}$-Anomer (7c): $[\alpha]{ }^{19} \mathrm{D}-174\left(c 1.05, \mathrm{CH}_{2} \mathrm{Cl}_{2}\right) ;{ }^{1} \mathrm{H} \mathrm{NMR}\left(600 \mathrm{MHz}, \mathrm{CDCl}_{3}\right) \delta 1.3$ (s, 3H), 1.56 (s, $3 \mathrm{H}), 3.66(\mathrm{~d}, J=7 \mathrm{~Hz}, 1 \mathrm{H}), 3.71$ (s, 3H), 3.84-3.90 (dd, $J=13,3 \mathrm{~Hz}, 1 \mathrm{H}), 3.87-3.93$ (br d, $J=13 \mathrm{~Hz}$, $1 \mathrm{H}), 4.03-4.09$ (dd, $J=13 \mathrm{~Hz}, 3 \mathrm{~Hz}, 1 \mathrm{H}), 4.05-4.12$ (br d, $J=13 \mathrm{~Hz}, 1 \mathrm{H}), 4.19$ (m, $1 \mathrm{H}$ ), 4.36 (app t, $J$ $=6 \mathrm{~Hz}, 1 \mathrm{H}), 6.85(\mathrm{~d}, J=9 \mathrm{~Hz}, 1 \mathrm{H}), 6.91(\mathrm{~d}, J=9 \mathrm{~Hz}, 1 \mathrm{H}), 7.17(\mathrm{dd}, J=8,1 \mathrm{~Hz}, 1 \mathrm{H}), 7.22(\mathrm{dd}, J=8$ $\mathrm{Hz}, 1 \mathrm{~Hz}), 7.32$ (dd, $J=8 \mathrm{~Hz}, 1 \mathrm{~Hz}, 1 \mathrm{H}), 7.36$ (dd, $J=7,1 \mathrm{~Hz}, 1 \mathrm{H}), 7.54$ (app t, $J=7 \mathrm{~Hz}, 2 \mathrm{H}), 7.61$ (d, $J=9 \mathrm{~Hz}, 1 \mathrm{H}), 7.64(\mathrm{~d}, J=9 \mathrm{~Hz}, 1 \mathrm{H}), 7.76(\mathrm{~d}, J=8 \mathrm{~Hz}, 1 \mathrm{H}), 7.81(\mathrm{~d}, J=8 \mathrm{~Hz}, 1 \mathrm{H}), 8.68(\mathrm{br} \mathrm{d}, J=6$ $\mathrm{Hz}, 1 \mathrm{H}), 8.98(\mathrm{~d}, J=6 \mathrm{~Hz}, 1 \mathrm{H}), 14.3(\mathrm{br} \mathrm{s}, 1 \mathrm{H}), 14.5$ (d, $J=6 \mathrm{~Hz}, 1 \mathrm{H}) ;{ }^{13} \mathrm{C} \mathrm{NMR}\left(150 \mathrm{MHz}, \mathrm{CDCl}_{3}\right)$

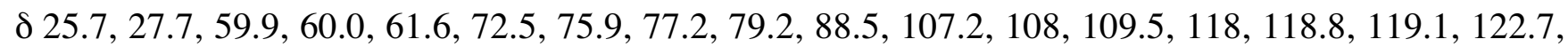
$122.9,123.3,124.3,126.3,126.7,127.9,128.0,128.9,129.0,133.3,133.4,137.3,137.5,156.5,160.2$, 172.4, 175.8; HRMS (FAB, 3-NOBA) calcd for $\mathrm{C}_{32} \mathrm{H}_{32} \mathrm{O}_{6} \mathrm{~N}_{2}(\mathrm{M}+\mathrm{H})^{+}$541.2340, obsd 541.2321.

$\alpha$-Anomer (6c): ${ }^{1} \mathrm{H}$ NMR (400 MHz, $\mathrm{CDCl}_{3}$ ) $\delta 1.27$ (s, 3H), 1.34 (s, 3H), 3.65-3.75 (m, 5H), 3.81 (dd, $J=12,6.3 \mathrm{~Hz}, 1 \mathrm{H}), 4.05(\mathrm{br} \mathrm{d}, J=13 \mathrm{~Hz}, 1 \mathrm{H}), 4.20(\mathrm{dd}, J=12,4.7 \mathrm{~Hz}, 1 \mathrm{H}), 4.36(\operatorname{app~t}, J=6.9 \mathrm{~Hz}$, $1 \mathrm{H}), 4.45(\mathrm{~m}, 1 \mathrm{H}), 6.83(\mathrm{~d}, J=9.4 \mathrm{~Hz}, 1 \mathrm{H}), 7.1(\mathrm{~d}, J=9.0 \mathrm{~Hz}, 1 \mathrm{H}), 7.29(\operatorname{app~t}, J=7.4 \mathrm{~Hz}, 1 \mathrm{H}), 7.35$ $(\operatorname{app~t}, J=7.6 \mathrm{~Hz}, 1 \mathrm{H}), 7.46(\operatorname{app~t}, J=7.6 \mathrm{~Hz}, 1 \mathrm{H}), 7.52(\mathrm{~d}, J=7.8 \mathrm{~Hz}, 1 \mathrm{H}), 7.6(\mathrm{~d}, J=9.4 \mathrm{~Hz}, 1 \mathrm{H})$, $7.68(\mathrm{~d}, J=7.9 \mathrm{~Hz}, 1 \mathrm{H}), 7.75(\mathrm{~d}, J=8.3 \mathrm{~Hz}, 1 \mathrm{H}), 7.76(\mathrm{~d}, J=9.1 \mathrm{~Hz}, 1 \mathrm{H}), 8.06(\mathrm{~d}, J=8.5 \mathrm{~Hz}, 1 \mathrm{H}), 8.6$ (br s, 1H), 9.4 (s, 1H), 14.1 (br s, 1H), 14.8 (s, 1H); ${ }^{13} \mathrm{C} \mathrm{NMR} \mathrm{(150} \mathrm{MHz,} \mathrm{CDCl}_{3}$ ) $\delta 24.4,26.6,57.1$, $60.7,62.8,71.5,74.9,83.2,90.5,106.8,108.3,109.8,117.9,119.5,120.1,122.8,123.3,124.9,126.2$, $127.5,127.8,127.9,128.9,129.1,133,133.8,135.2,137.8,157.6,159.4,164.4,174.8,177.3$; HRMS (FAB, 3-NOBA) calcd for $\mathrm{C}_{32} \mathrm{H}_{32} \mathrm{O}_{6} \mathrm{~N}_{2}(\mathrm{M}+\mathrm{H})^{+}$541.2339, obsd 541.2330.

\section{$N, N^{\prime}$-Bis(1'-hydroxy-2'-naphthylidene)-3- $O$-methyl-4,5- $O$-isopropylidene-1,2-dideoxy- $\beta$-D-}

fructopyranosyl-1,2-diamine (7d). : General Procedure A was followed, beginning from anomeric diamines 6/7 (162 mg, $0.700 \mathrm{mmol})$ and 1-hydroxy-2-naphthaldehyde (241 mg, $1.40 \mathrm{mmol})$, with overnight heating at $50{ }^{\circ} \mathrm{C}$. $\mathrm{SiO}_{2}$ column chromatography (35\% EtOAc-hexanes), yielded pure 7d (188 mg, 50\%): $[\alpha]^{19} \mathrm{D}^{-846}\left(c 0.97, \mathrm{CH}_{2} \mathrm{Cl}_{2}\right) ;{ }^{1} \mathrm{H} \mathrm{NMR}\left(600 \mathrm{MHz}, \mathrm{CDCl}_{3}\right) \delta 1.33(\mathrm{~s}, 3 \mathrm{H}), 1.53(\mathrm{~s}, 3 \mathrm{H})$, $3.65(\mathrm{~d}, J=6.8 \mathrm{~Hz}, 1 \mathrm{H}), 3.72(\mathrm{~s}, 3 \mathrm{H}), 3.76(\mathrm{br} \mathrm{d}, J=13 \mathrm{~Hz}, 1 \mathrm{H}), 3.87$ (dd, $J=13,3 \mathrm{~Hz}, 1 \mathrm{H}), 3.98$ (br d, 
$J=13 \mathrm{~Hz}, 1 \mathrm{H}), 4.05(\mathrm{dd}, J=13,3 \mathrm{~Hz}, 1 \mathrm{H}), 4.2(\mathrm{~m}, 1 \mathrm{H}), 4.39(\operatorname{app~t}, J=7 \mathrm{~Hz}, 1 \mathrm{H}), 6.79(\mathrm{~d}, J=9 \mathrm{~Hz}$, $1 \mathrm{H}), 6.86(\mathrm{~d}, J=9 \mathrm{~Hz}, 2 \mathrm{H}), 6.90(\mathrm{~d}, J=9 \mathrm{~Hz}, 1 \mathrm{H}), 7.41-7.45(\mathrm{~m}, 2 \mathrm{H}), 7.53-7.59$ (m, 4H), $7.77(\mathrm{~d}, J=$ $5 \mathrm{~Hz}, 1 \mathrm{H}), 8.10(\mathrm{~d}, J=7 \mathrm{~Hz}, 1 \mathrm{H}), 8.43(\mathrm{dd}, J=8,4.8 \mathrm{~Hz}, 1 \mathrm{H}), 13.41$ (br s, 1H), $13.70(\mathrm{~d}, J=7 \mathrm{~Hz}$, $1 \mathrm{H}) ;{ }^{13} \mathrm{C} \mathrm{NMR}\left(150 \mathrm{MHz}, \mathrm{CDCl}_{3}\right) \delta 25.7,27.6,59.5,60.0,61.9,72.5,76,79.1,88.3,109.7,109.8$, 110.6, 115.7, 116.8, 125.0, 125.2, 125.3, 125.4, 127.2, 127.3, 127.7, 127.9, 128.2, 129.2, 130.2, 130.2, 137.2, 137.4, 159.4, 163.4, 171.9, 174.8; HRMS (FAB, 3-NOBA) calcd for $\mathrm{C}_{32} \mathrm{H}_{32} \mathrm{~N}_{2} \mathrm{O}_{6}(\mathrm{M}+\mathrm{H})^{+}$ 541.2340, obsd 541.2320.

\section{$N, N^{\prime}$-Bis(3',5'-diiodosalicylidene)-3- $O$-methyl-4,5- $O$-isopropylidene-1,2-dideoxy- $\beta$-D-}

fructopyranosyl-1,2-diamine (7e). Following General Procedure A, heating overnight at $50{ }^{\circ} \mathrm{C}$, from anomeric diamines $6 / 7(162 \mathrm{mg}, 0.700 \mathrm{mmol})$ and 3,5-diiodosalicylaldehyde (241 $\mathrm{mg}, 1.40 \mathrm{mmol})$, with subsequent $\mathrm{SiO}_{2}$ column chromatography $\left(25 \rightarrow 50 \% \mathrm{Et}_{2} \mathrm{O}\right.$-hexanes), was obtained $7 \mathrm{e}(212 \mathrm{mg}$,

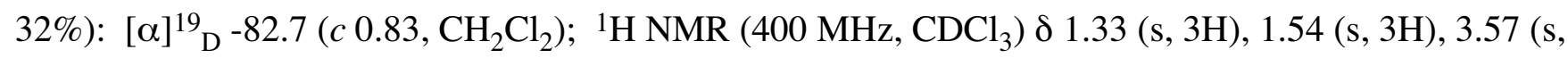
$3 \mathrm{H}), 3.81-3.90(\mathrm{~m}, 2 \mathrm{H}), 4.04(\mathrm{~d}, J=12.3 \mathrm{~Hz}, 1 \mathrm{H}), 4.16-4.19(\mathrm{~m}, 1 \mathrm{H}), 4.29(\operatorname{app~t}, J=6.3 \mathrm{~Hz}, 1 \mathrm{H})$, $7.48(\mathrm{~d}, J=2 \mathrm{~Hz}, 1 \mathrm{H}), 7.62(\mathrm{~d}, J=2 \mathrm{~Hz}, 1 \mathrm{H}), 8.04(\mathrm{~d}, J=2 \mathrm{~Hz}, 1 \mathrm{H}), 8.06-8.07(\mathrm{~m}, 2 \mathrm{H}), 8.33(\mathrm{~s}, 1 \mathrm{H})$, 14.10 (s, 1H), 14.30 (br s, 1H); $\left.{ }^{13} \mathrm{C} \mathrm{NMR} \mathrm{(150} \mathrm{MHz,} \mathrm{CDCl}_{3}\right) \delta$ 25.2, 27.2, 60.4, 62.7, 63.9, 72.1, 75, 79, 79.1, 79.9, 87.3, 88.2, 90.2, 109.7, 119.6, 119.8, 140.1, 140.9, 149.1, 149.2, 160.2, 161.4, 162.0, 166.1; HRMS (FAB, 3-NOBA) calcd for $\mathrm{C}_{24} \mathrm{H}_{24} \mathrm{~N}_{2} \mathrm{O}_{6} \mathrm{I}_{4}(\mathrm{M}+\mathrm{H})^{+} 944.7893$, obsd 944.7869.

\section{$N, N^{\prime}$-Bis(4'-benzyloxysalicylidene)-3- $O$-methyl-4,5- $O$-isopropylidene-1,2-dideoxy- $\beta$-D-}

fructopyranosyl-1,2-diamine (7f). Following General Procedure A, heating overnight at $50{ }^{\circ} \mathrm{C}$, from anomeric diamines 6/7 (162 mg, $0.700 \mathrm{mmol})$ and 4-benzyloxysalicylaldehyde (319 $\mathrm{mg}, 1.40 \mathrm{mmol})$, with subsequent $\mathrm{SiO}_{2}$ column chromatography $(25 \rightarrow 50 \%$ EtOAc-hexanes), was obtained $7 \mathbf{f}(230 \mathrm{mg}$, 48\%): $[\alpha]{ }^{19}{ }_{\mathrm{D}}-188\left(c 1.00, \mathrm{CH}_{2} \mathrm{Cl}_{2}\right) ;{ }^{1} \mathrm{H} \mathrm{NMR}$ (600 MHz, $\left.\mathrm{CDCl}_{3}\right) \delta 1.32$ (s, 3H), $1.54(\mathrm{~s}, 3 \mathrm{H}), 3.61$ (s, $3 \mathrm{H}), 3.67(\mathrm{~d}, J=13 \mathrm{~Hz}, 1 \mathrm{H}), 3.7(\mathrm{~d}, J=7 \mathrm{~Hz}, 1 \mathrm{H}), 3.9$ (d, $J=3 \mathrm{~Hz}, 1 \mathrm{H}), 3.97(\mathrm{~d}, J=13 \mathrm{~Hz}, 1 \mathrm{H}), 4.15-$ $4.17(\mathrm{~m}, 1 \mathrm{H}), 4.27(\mathrm{t}, J=7 \mathrm{~Hz}, 1 \mathrm{H}), 5.06(\mathrm{~s}, 1 \mathrm{H}), 5.07(\mathrm{~s}, 1 \mathrm{H}), 6.45(\mathrm{dd}, J=9,2 \mathrm{~Hz}, 1 \mathrm{H}), 6.49-6.52(\mathrm{~m}$, $3 \mathrm{H}), 7.1(\mathrm{~d}, J=9 \mathrm{~Hz}, 1 \mathrm{H}), 7.19(\mathrm{~d}, J=8 \mathrm{~Hz}, 1 \mathrm{H}), 7.31(\operatorname{app~t}, J=7 \mathrm{~Hz}, 2 \mathrm{H}), 7.38(\operatorname{app~t}, J=7 \mathrm{~Hz}, 4 \mathrm{H})$, $7.41(\mathrm{~d}, J=7 \mathrm{~Hz}, 2 \mathrm{H}), 8.2(\mathrm{~s}, 1 \mathrm{H}), 8.4(\mathrm{~s}, 1 \mathrm{H}), 13.5(\mathrm{~m}, 2 \mathrm{H}) ;{ }^{13} \mathrm{C} \mathrm{NMR}\left(150 \mathrm{MHz}, \mathrm{CDCl}_{3}\right) \delta 25.5$, 27.5, 60.3, 62.0, 653.7, 69.9, 70.0, 72.6, 76.0, 79.5, 89.9, 102.1, 107.1, 107.3, 109.2, 112.5, 127.4, $127.5,128,128.1,128.5,128.6,132.9,133.6,136.3,136.4,162.9,163.0,164.2,165.5,166.5$; HRMS (FAB, 3-NOBA) calcd for $\mathrm{C}_{38} \mathrm{H}_{40} \mathrm{O}_{8} \mathrm{~N}_{2}(\mathrm{M}+\mathrm{H})^{+}$653.2863, obsd 653.2875. 
General Procedure B: Synthesis of Cobalt-(III)-Salen Complexes. To a stirred solution of 'salen' ligand (7e) (43 mg, $0.045 \mathrm{mmol}, 1$ equiv.) in $5 \mathrm{~mL} \mathrm{CH}_{2} \mathrm{Cl}_{2}$, a methanolic $(2 \mathrm{~mL})$ solution of cobalt (II) acetate tetrahydrate $(11.4 \mathrm{mg}, 45 \mu \mathrm{mol}, 1.0$ equiv.), was added via cannula, under Ar. The Co(II)-salen complex precipitated out as a red solid. After filtration, the $\mathrm{Co}$ (II) complex was taken up in $\mathrm{CH}_{2} \mathrm{Cl}_{2}$ or toluene $(2 \mathrm{~mL})$ and stirred with acetic acid (>10 equiv.) [or 3,5-dinitrobenzoic acid (1 equiv.)] open to the air. The oxidation could be followed by TLC [formation of a greenish-brown spot of lower $\mathrm{R}_{\mathrm{f}}\{\mathrm{Co}-$ (III) complex\} from the visibly red, higher $\mathrm{R}_{\mathrm{f}}$ spot characteristic of the Co-(II) salen]. When TLC indicated the completion of the reaction (2-12 h), the solvent was evaporated, and the Co(III)-salen complex further dried in vacuo. The Co(III) carboxylate complexes so prepared were generally used directly for HKR experiments, under ISES (bilayer) conditions or under neat 'Jacobsen conditions. ${ }^{7}$

Table S1: MS characterization of Co(III)-salen catalysts.

\begin{tabular}{|c|c|c|c|}
\hline Catalyst & Molecular Formula & Calcd & obsd MS (FAB, 3-NOBA) \\
\hline 1b & $\mathrm{C}_{34} \mathrm{H}_{45} \mathrm{~N}_{2} \mathrm{O}_{4} \mathrm{Co}$ & 545.3 & 545.2 \\
\hline 1c & $\mathrm{C}_{34} \mathrm{H}_{33} \mathrm{~N}_{2} \mathrm{O}_{4} \mathrm{Co}$ & 533.2 & 533.1 \\
\hline 1f & $\mathrm{C}_{40} \mathrm{H}_{38} \mathrm{~N}_{2} \mathrm{O}_{6} \mathrm{Co}$ & 645.2 & 645.1 \\
\hline $1 \mathrm{~g}$ & $\mathrm{C}_{46} \mathrm{H}_{63} \mathrm{~N}_{4} \mathrm{O}_{4} \mathrm{Co}$ & 735.4 & 735.3 \\
\hline $2 a$ & $\mathrm{C}_{40} \mathrm{H}_{53} \mathrm{~N}_{2} \mathrm{O}_{4} \mathrm{Co}$ & 625.3 & 625.4 \\
\hline $2 \mathbf{b}$ & $\mathrm{C}_{32} \mathrm{H}_{37} \mathrm{~N}_{2} \mathrm{O}_{4} \mathrm{Co}$ & 513.2 & 513.2 \\
\hline $2 c$ & $\mathrm{C}_{32} \mathrm{H}_{25} \mathrm{~N}_{2} \mathrm{O}_{4} \mathrm{Co}$ & 501.1 & 501.1 \\
\hline 2d & $\mathrm{C}_{32} \mathrm{H}_{25} \mathrm{~N}_{2} \mathrm{O}_{4} \mathrm{Co}$ & 501.1 & 501.1 \\
\hline $2 f$ & $\mathrm{C}_{38} \mathrm{H}_{33} \mathrm{~N}_{2} \mathrm{O}_{6} \mathrm{Co}$ & 613.2 & 613.2 \\
\hline $2 \mathrm{~g}$ & $\mathrm{C}_{44} \mathrm{H}_{55} \mathrm{~N}_{4} \mathrm{O}_{4} \mathrm{Co}$ & 703.3 & 703.3 \\
\hline 3a & $\mathrm{C}_{41} \mathrm{H}_{55} \mathrm{~N}_{2} \mathrm{O}_{4} \mathrm{Co}$ & 639.3 & 639.4 \\
\hline $3 \mathbf{b}$ & $\mathrm{C}_{33} \mathrm{H}_{39} \mathrm{~N}_{2} \mathrm{O}_{4} \mathrm{Co}$ & 527.2 & 527.2 \\
\hline $3 c$ & $\mathrm{C}_{33} \mathrm{H}_{27} \mathrm{~N}_{2} \mathrm{O}_{4} \mathrm{Co}$ & 516.1 & 516.1 \\
\hline 3d & $\mathrm{C}_{33} \mathrm{H}_{27} \mathrm{~N}_{2} \mathrm{O}_{4} \mathrm{Co}$ & 516.1 & 516.1 \\
\hline $3 e$ & $\mathrm{C}_{25} \mathrm{H}_{19} \mathrm{~N}_{2} \mathrm{O}_{4} \mathrm{I}_{4} \mathrm{Co}$ & 918.7 & 918.7 \\
\hline $3 f$ & $\mathrm{C}_{39} \mathrm{H}_{35} \mathrm{~N}_{2} \mathrm{O}_{6} \mathrm{Co}$ & 627.2 & 627.2 \\
\hline $6 \mathbf{a}$ & $\mathrm{C}_{42} \mathrm{H}_{61} \mathrm{~N}_{2} \mathrm{O}_{8} \mathrm{Co}$ & 721.4 & 721.3 \\
\hline $6 \mathbf{b}$ & $\mathrm{C}_{34} \mathrm{H}_{45} \mathrm{~N}_{2} \mathrm{O}_{8} \mathrm{Co}$ & 609.2 & 609.2 \\
\hline $6 c$ & $\mathrm{C}_{34} \mathrm{H}_{33} \mathrm{~N}_{2} \mathrm{O}_{8} \mathrm{Co}$ & 597.1 & 597.0 \\
\hline $7 \mathbf{a}$ & $\mathrm{C}_{42} \mathrm{H}_{61} \mathrm{~N}_{2} \mathrm{O}_{8} \mathrm{Co}$ & 721.4 & 721.3 \\
\hline $7 \mathrm{~b}$ & $\mathrm{C}_{34} \mathrm{H}_{45} \mathrm{~N}_{2} \mathrm{O}_{8} \mathrm{Co}$ & 609.2 & 609.3 \\
\hline $7 \mathrm{c}$ & $\mathrm{C}_{34} \mathrm{H}_{33} \mathrm{~N}_{2} \mathrm{O}_{8} \mathrm{Co}$ & 597.1 & 597.0 \\
\hline 7d & $\mathrm{C}_{34} \mathrm{H}_{33} \mathrm{~N}_{2} \mathrm{O}_{8} \mathrm{Co}$ & 597.1 & 597.2 \\
\hline $7 f$ & $\mathrm{C}_{40} \mathrm{H}_{41} \mathrm{~N}_{2} \mathrm{O}_{10} \mathrm{Co}$ & 709.2 & 709.0 \\
\hline
\end{tabular}




\section{Enzyme Standardization}

A. Stock Solutions. The following stock solutions were made for enzyme standardization: $220 \mathrm{mM}$ $\beta-\mathrm{NAD}^{+}, 220 \mathrm{mM} \beta-\mathrm{NADP}^{+}$, alcohol dehydrogenases from horse liver (0.036 nominal EtOH units/ $\left.\mu \mathrm{L}\right)$ and T. brockii $(0.147$ nominal i-PrOH units/ $\mu \mathrm{L})$ in $25 \mathrm{mM}$ sodium phosphate buffer, $\mathrm{pH} 7.0$ and $2 \mathrm{M}$ (R)-1,2-propanediol in $\mathrm{H}_{2} \mathrm{O}$. Enzyme units were calculated by measuring the rate of formation of $\mathrm{NAD}(\mathrm{P}) \mathrm{H}$ at $340 \mathrm{~nm}$ (vide infra). In each case, one S.I. unit is taken as the amount of enzyme catalyzing the formation of one $\mu \mathrm{mol}$ of $\mathrm{NAD}(\mathrm{P}) \mathrm{H}$ per minute.

B. Standardization of TBADH: The assay cuvet contained the following components: $2.2 \mathrm{mM}$ (10 $\mu \mathrm{L} 220 \mathrm{mM}$ stock) $\mathrm{NADP}^{+}, 2 \mu \mathrm{L}$ of TBADH stock solution, $888 \mu \mathrm{L}$ of $50 \mathrm{mM}$ sodium pyrophosphate buffer, $\mathrm{pH} 8.8$, and $200 \mathrm{mM}$ (100 $\mu \mathrm{L}$ of $2 \mathrm{M}$ stock) of $(R)$-1,2-propanediol. The reaction was initiated by the addition of the $(R)$-1,2-propanediol, which typically gave a rate of $0.275 \pm 0.001 \mathrm{Abs} / \mathrm{min}$ at $25^{\circ} \mathrm{C}$, $340 \mathrm{~nm}$. This was indicative of $0.022 \mathrm{U}$ of TBADH per $\mu \mathrm{L}$ of the stock solution.

C. Standardization of HLADH: The assay cuvet contained the following components: $7.2 \mathrm{mM}$ (33 $\mu \mathrm{L}$ of $220 \mathrm{mM}$ stock) of $\mathrm{NAD}^{+}, 2 \mu \mathrm{L}$ of HLADH stock solution, $865 \mu \mathrm{L}$ of $50 \mathrm{mM}$ sodium pyrophosphate buffer, $\mathrm{pH} 8.8$, and $200 \mathrm{mM}(100 \mu \mathrm{L}$ of $2 \mathrm{M}$ stock) of $(R)$-1,2-propanediol. The reaction was initiated by the addition of the $(R)$-1,2-propanediol, which typically gave a rate of $0.25 \pm 0.01$ Abs/min at $25^{\circ} \mathrm{C}, 340 \mathrm{~nm}$. This was indicative of $0.020 \mathrm{U}$ of HLADH per $\mu \mathrm{L}$ of the stock solution.

\section{Dehydrogenase Enantioselectivities}

\section{A. Relative Velocity $\left(\mathrm{v}_{\text {rel }}\right)$ Determinations}

1. TBADH: The enantioselectivity of TBADH was estimated from the ratios of the initial velocities of $(R)$ - vs. $(S)$-1,2-propanediol at fixed concentrations between 3.0 and $80 \mathrm{mM}$ at $25^{\circ} \mathrm{C}$. Each velocity measurement was performed in triplicate. The assay cuvet contained the following: $2.2 \mathrm{mM}$ $\mathrm{NADP}^{+}(10 \mu \mathrm{L}$ from a $220 \mathrm{mM}$ stock), $0.113 \mathrm{U}$ of TBADH, and various concentrations of either $(R)$ or $(S)$-enantiomer of 1,2-propanediol. In all cases, the final volume was adjusted to $1 \mathrm{~mL}$ using $50 \mathrm{mM}$ sodium pyrophosphate buffer, $\mathrm{pH}$ 8.8. [Note: The concentration of NADP+ is fixed at saturating levels here, as reported $\mathrm{K}_{\mathrm{m}}$ value for $\mathrm{NADP}^{+}$with $\mathrm{TBADH}$ is $\left.13 \mu \mathrm{M}^{8}{ }^{8}\right]$

2. HLADH: The enantioselectivity of TBADH was estimated from the ratios of the initial velocities of $(R)$ - vs. $(S)$-1,2-propanediol at fixed concentrations between 4.0 and $100 \mathrm{mM}$ at $25{ }^{\circ} \mathrm{C}$. Each velocity measurement was made in duplicate. The assay cuvet contained the following: $7.2 \mathrm{mM}$ $\mathrm{NAD}^{+}(33 \mu \mathrm{L}$ from a $220 \mathrm{mM}$ stock), $0.049 \mathrm{U}$ of HLADH, and various concentrations of either the $(R)$ or $(S)$-enantiomer of 1,2-propanediol. In all cases, the final volume was adjusted to $1 \mathrm{~mL}$ using $50 \mathrm{mM}$ sodium pyrophosphate buffer, $\mathrm{pH}$ 8.8. [Note: The concentration of $\mathrm{NAD}^{+}$is fixed at saturating levels here, as reported $\mathrm{K}_{\mathrm{m}}$ values for $\mathrm{NAD}^{+}$with $\mathrm{HLADH}$ range from $3^{9}$ to $62^{8} \mu \mathrm{M}$.]

The data are collected in Figure S1 and in Table S2 below. One sees that TBADH displays a significant preference for the $(R)$-1,2-propanediol, and that HLADH favors the $(S)$-antipode, though 
not to the same degree. For each dehydrogenase, the relative velocities are relatively flat as a function of diol concentration. In each case, there is a slight increase in enantioselectivity as one goes to lower concentration, indicating that each enzyme displays the same enantiomeric preference in the binding step (i.e. lower $\mathrm{K}_{\mathrm{m}}$ ) as in the catalytic step (higher $\mathrm{k}_{\text {cat }}$ or $\mathrm{V}_{\max }$ ).

Figure S1: Dehydrogenase Enantiopreferences vs. 1,2-Propanediol Concentration

\section{A. HLADH}

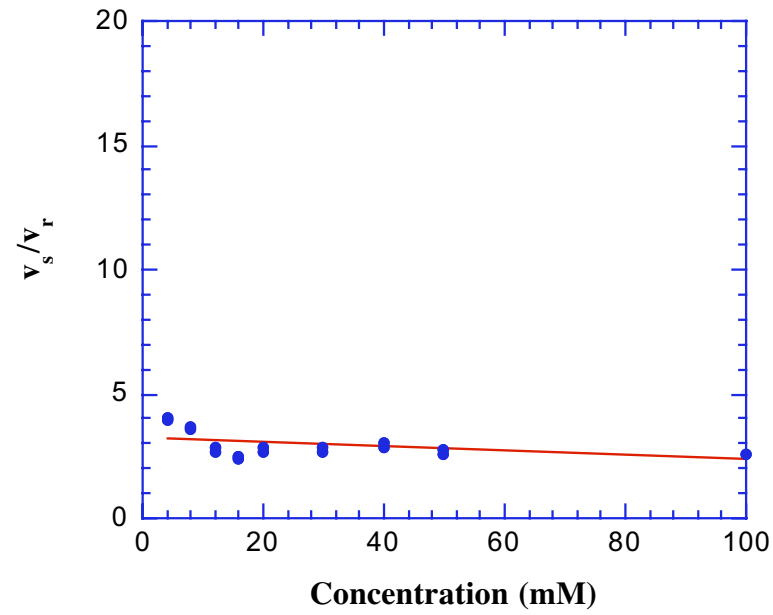

\section{B. TBADH}

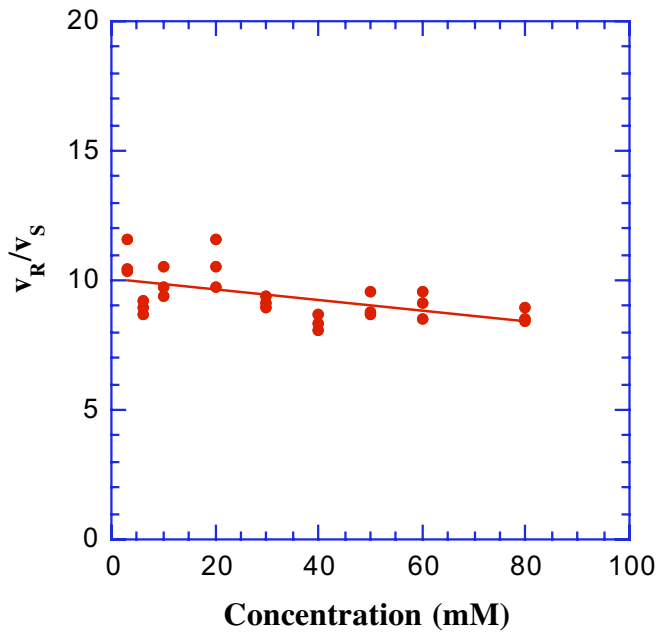

Table S2: Tabulated Dehydrogenase Enantioselectivities vs. Diol Concentration

\begin{tabular}{|c|c|c|c|c|c|c|c|c|c|}
\hline $\begin{array}{c}\text { 1,2-Propanediol } \\
\text { Conc. }(\mathrm{mM})\end{array}$ & 4 & 8 & 12 & 16 & 20 & 30 & 40 & 50 & 100 \\
\hline $\begin{array}{c}\mathrm{v}_{\mathrm{S}} / \mathrm{v}_{\mathrm{R}} \text { of } \\
\mathrm{HLADH}\end{array}$ & 4.0 & 3.6 & 2.8 & 2.4 & 2.7 & 2.8 & 3.0 & 2.7 & 2.6 \\
\hline
\end{tabular}

\begin{tabular}{|c|c|c|c|c|c|c|c|c|c|}
\hline $\begin{array}{c}\text { 1,2-Propanediol } \\
\text { Conc. }(\mathrm{mM})\end{array}$ & 3 & 6 & 10 & 20 & 30 & 40 & 50 & 60 & 80 \\
\hline $\begin{array}{c}\mathrm{v}_{\mathrm{R}} / \mathrm{v}_{\mathrm{S}} \text { of } \\
\text { TBADH }\end{array}$ & 10.8 & 8.9 & 9.9 & 10.6 & 9.1 & 8.4 & 9.0 & 9.1 & 8.6 \\
\hline
\end{tabular}

The flatness of each graph suggests that $\mathrm{K}_{\mathrm{m}}$ 's for the two enantiomers are likely not very different with either dehydrogenase. Indeed, this was confirmed by the determination of $\mathrm{K}_{\mathrm{m}}$ 's for each antipode of 1,2-propanediol for each enzyme (vide infra). 


\section{B. Michaelis-Menten Kinetic Analysis: $K_{\mathrm{m}}$ and $\mathbf{V}_{\max }$ Determinations}

Figure S2
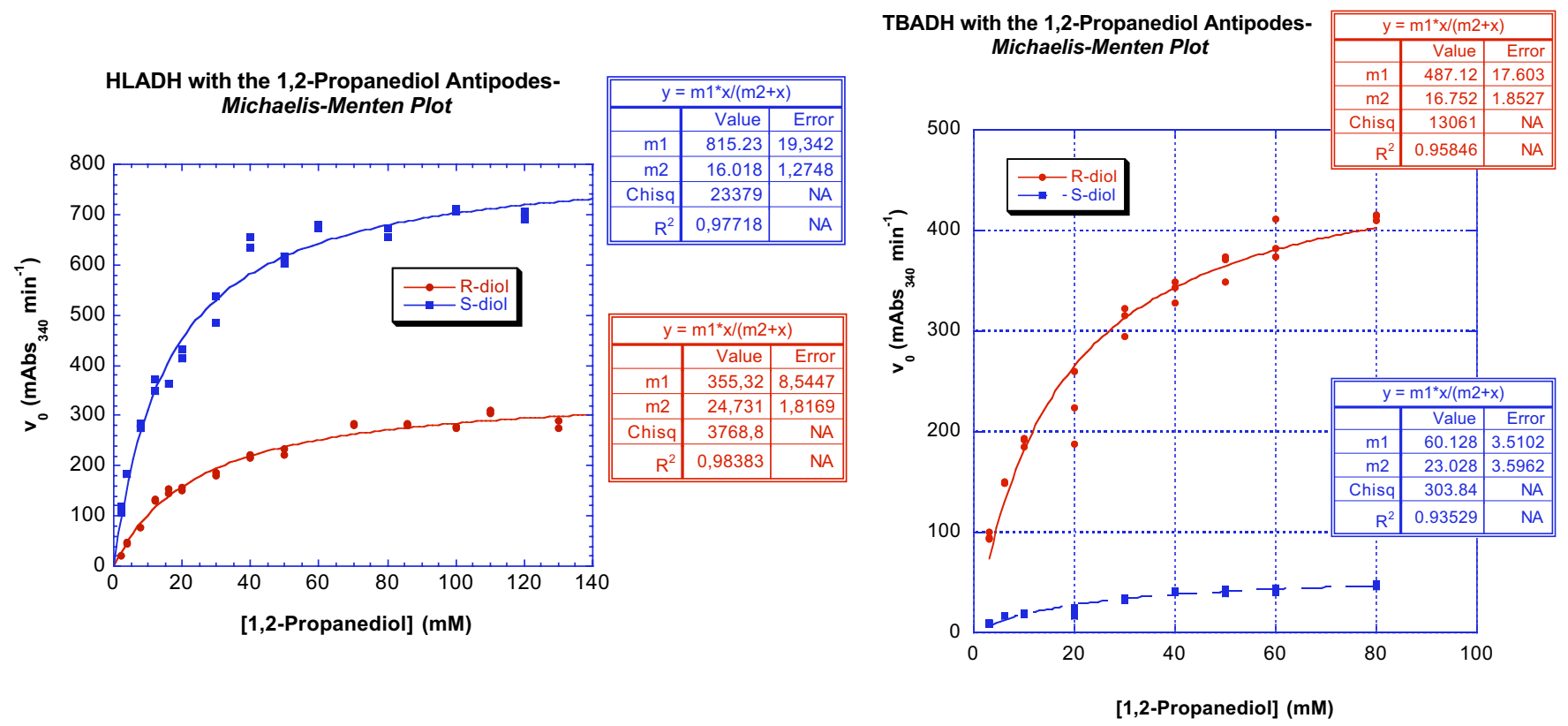

For each antipodal diol, the same data as were used to survey dehydrogenase enantioselectivity, as a function of diol concentration, were plotted in Michaelis-Menten fashion, as $\mathrm{v}_{0}$ vs. [S] (Figure S2, above). For each dehydrogenase, the data for $(R)$-1,2-propanediol are shown in red, and those for the $(S)$-enantiomer are in blue. In all four cases, the data were processed by linear, least squares fitting to a hyperbolic function. The resulting curve fits and best fit hyperbolic equations are presented above.

For HLADH, we obtain the following steady state kinetic parameters:

$$
\begin{aligned}
& (R) \text {-1,2-propanediol }-\mathrm{K}_{\mathrm{m}}=25 \pm 2 \mathrm{mM} \text { and } \mathrm{V}_{\max }=355 \pm 9 \mathrm{mAbs} \mathrm{min}^{-1} \mathrm{vs} . \\
& (S) \text {-1,2-propanediol }-\mathrm{K}_{\mathrm{m}}=16 \pm 1 \mathrm{mM} \text { and } \mathrm{V}_{\max }=819 \pm 20 \mathrm{mAbs} \mathrm{min}^{-1}
\end{aligned}
$$

Translating these data into the ratio of catalytic efficiencies for the two enantiomers, one obtains $\left[\left(\mathrm{V}_{\max } / \mathrm{K}_{\mathrm{m}}\right)_{\mathrm{S}} \div\left(\mathrm{V}_{\max } / \mathrm{K}_{\mathrm{m}}\right)_{\mathrm{R}}\right]=3.6$. This is consistent with the trends seen in enantioselectivity vs. concentration, as the HLADH enantioselectivity tends toward $\sim 4: 1(S: R)$ as one tends toward low diol concentrations (see Table S2 and Figure S1, above).

For TBADH, we obtain the following kinetic constants:

$$
\begin{aligned}
& (R) \text {-1,2-propanediol }-\mathrm{K}_{\mathrm{m}}=17 \pm 2 \mathrm{mM} \text { and } \mathrm{V}_{\max }=487 \pm 18 \mathrm{mAbs} \mathrm{min}^{-1} \mathrm{vs} . \\
& (S) \text {-1,2-propanediol }-\mathrm{K}_{\mathrm{m}}=23 \pm 4 \mathrm{mM} \text { and } \mathrm{V}_{\max }=60 \pm 4 \mathrm{mAbs}_{\min }{ }^{-1}
\end{aligned}
$$

Here the ratio of catalytic efficiencies for the two enantiomeric diols is dominated by the $\mathrm{V}_{\max }$ terms and yields $\left[\left(\mathrm{V}_{\text {max }} / \mathrm{K}_{\mathrm{m}}\right)_{\mathrm{R}} \div\left(\mathrm{V}_{\max } / \mathrm{K}_{\mathrm{m}}\right)_{\mathrm{S}}\right]=11$. Again, one sees nice agreement with trends observed in the enantioselectivity vs. concentration data, where the enantioselectivity tends toward $~$ 11:1 $(R: S)$ as one tends toward low diol concentrations (see Table S2 and Figure S1, above). 


\section{Double Cuvet-ISES (In Situ Enzymatic Screening) Procedure}

A general procedure for 'double cuvet-ISES' to estimate both enantioselectivities and relative rates for an array of catalysts being screened in parallel is presented below. The hydrolytic kinetic resolution $(\mathrm{HKR})$ of $( \pm)$ propylene oxide was chosen as model reaction. An inverted bilayer with $\mathrm{CHCl}_{3}$ as the organic solvent was chosen for this study, though initial trials indicate that other solvents both more dense (alternative lower organic layers), and less dense (upper organic layer) than the aqueous reporting layer, will also work.

Typically, quartz cuvets with one cm light paths and with nominal one mL volumes were used. For each catalyst, a two cuvet screen was performed, with cuvet 1 containing TBADH, as a $(R)$ propanediol-selective reporting enzyme, and cuvet 2 containing HLADH, as $(S)$-propanediol-selective reporting enzyme. For each cuvet, the final volume was $800 \mu \mathrm{L}(300 \mu \mathrm{L}$ of organic layer; $500 \mu \mathrm{L}$ of aqueous layer). This experimental set-up sends the spectrophotometer light beam cleanly through the aqueous reporting layer, as control experiments established that the beam passes through $1 \mathrm{~mL}$ cuvets between the 600 and $700 \mu \mathrm{L}$ volume levels.

A. Organic Layer Composition: Both cuvets 1 and 2 had the following composition: $150 \mu \mathrm{L}$ $(2.15 \mathrm{mmol})$ of $\left( \pm\right.$ )-propylene oxide $\left(7.2 \mathrm{M}\right.$ in organic layer), $150 \mu \mathrm{L}$ of $\mathrm{CHCl}_{3}$ and $0.25 \mathrm{~mol} \% \mathrm{Co}$ (III)salen catalyst. The total organic layer volume was maintained as $300 \mu \mathrm{L}$ in all catalyst screens.

\section{B. Aqueous Layer Composition:}

(i) Cuvet 1: $0.35 \mathrm{U} \mathrm{TBADH}(15.8 \mu \mathrm{L}$ from a stock solution $(0.022 \mathrm{U} / \mu \mathrm{L}))$ of, $2.2 \mathrm{mM} \beta-\mathrm{NADP}^{+}$ (5 $\mu \mathrm{L}$ from a $220 \mathrm{mM}$ stock) and $479.5 \mu \mathrm{L}$ of $50 \mathrm{mM}$ sodium pyrophosphate buffer, $\mathrm{pH} 8.8$. The final $\mathrm{pH}$ was 8.6. The total volume of the aqueous layer was maintained as $500 \mu \mathrm{L}$ in both cuvets in all ISES experiments.

(ii) Cuvet 2: $0.35 \mathrm{U}$ HLADH $(17.6 \mu \mathrm{L}$ from a stock solution $(0.02 \mathrm{U} / \mu \mathrm{L})), 7.2 \mathrm{mM} \beta-\mathrm{NAD}^{+}$ (16.5 $\mu \mathrm{L}$ from a $220 \mathrm{mM}$ stock) and $465.8 \mu \mathrm{L}$ of $50 \mathrm{mM}$ sodium pyrophosphate buffer, $\mathrm{pH}$ 8.8. The final $\mathrm{pH}$ was 8.6.

C. Step by Step Protocol: A catalyst stock solution (22 $\mu \mathrm{mol}$ of catalyst in $620 \mu \mathrm{L} \mathrm{CHCl}_{3}$ ) was made up in a $1.5 \mathrm{ml}$ microcentrifuge tube, and stored on ice, along with a stock of ( \pm )-propylene oxide, in a separate tube. The organic layers were prepared by briefly vortexing $150 \mu \mathrm{L}(5.4 \mu \mathrm{mol}, 0.25$ mol\%) of the catalyst stock in $\mathrm{CHCl}_{3}$ with $150 \mu \mathrm{L}(2.15 \mathrm{mmol})$ of $( \pm)$-propylene oxide in iced microcentrifuge tubes. These were loaded into $1 \mathrm{~mL}$ quartz cuvets using precooled (refrigerator), disposable $1 \mathrm{~mL}$ syringes. The aqueous layers were then layered on top of the organic layers, by syringe, down the walls of the cuvet. [Note: A reversed loading method was adapted for the catalysts made using 1-hydroxy-2-naphthaldehyde (aldehyde 'd') in order to get a clear bilayer. Namely, it was found practical for these catalysts to load the aqueous layer first. Then, the organic layer was syringed beneath the aqueous layer.] Catalyst screens were routinely run done in duplicate (i.e. two runs of cuvet 1 and two of cuvet 2 per screen), with ISES rates taken as the average of the two $\Delta$ O.D.340/time values obtained, for the appropriate time window (vide infra). 


\section{Illustration of the Apparatus}

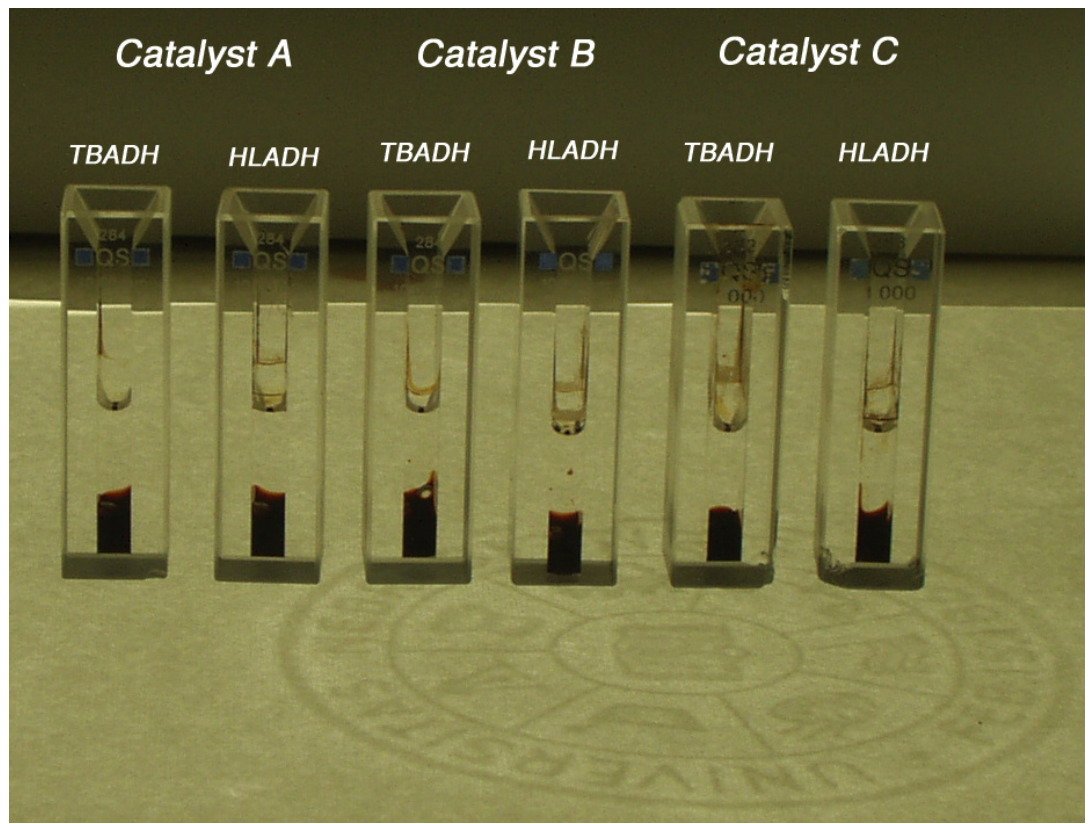

Cuvet Pairs Prepared for Screening

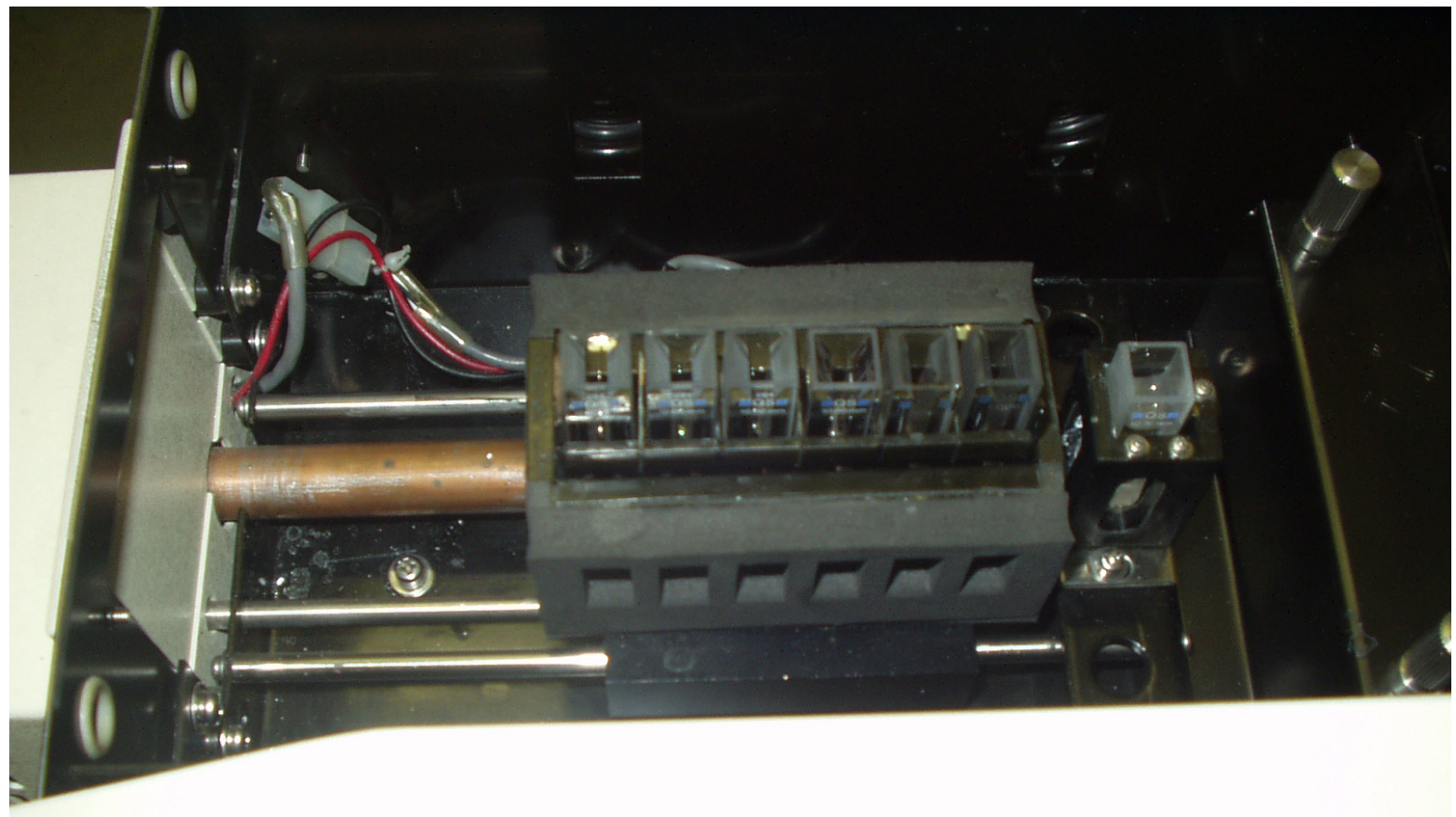

Cuvets loaded into a Shimadzu UV-2101PC Spectrophotometer with Cell Changer 


\section{Estimation of Enantioselectivity for Catalysts Screened}

Previously, in monitoring transition metal-mediated allylic substitution chemistry, ISES conditions were found in which a reliable signal could be obtained in 10-20 min. ${ }^{10}$ With the working principle of streamlining the data collection process, we set out to establish a screening protocol with a time window in this range here. Indeed, for 'fast' catalysts, it was found that a 15 minute time window sufficed to provide meaningful ISES rates in the two reporting cuvets, allowing for catalyst characterization. This time window was employed for all catalysts displaying rates surpassing 50 $\mathrm{mAbs} / \mathrm{min}$ (fastest rate of the two reporting cuvets). For catalysts exhibiting ISES rates $\leq 50 \mathrm{mAbs} / \mathrm{min}$ over the first 15 minutes, a longer observation window was chosen (35 minute total), so as to obtain a better signal. Generally, catalysts displaying ISES rates of less than $20 \mathrm{mAbs} / \mathrm{min}$ over 35 minutes were not pursued further.

The following expression was used to estimate catalyst enantioselectivity (see Appendix for derivation):

$$
\text { Catalyst Enantioselectivity }=\frac{[R]}{[S]}=\frac{\left[\left(\frac{\operatorname{Sel}_{E 2}}{\operatorname{Sel}_{E 1}}\right) \cdot v_{E 2}-v_{E 1}\right]}{\operatorname{Sel}_{E 2} \bullet\left(v_{E 1}-v_{E 2}\right)}
$$

- where the velocities in each of the reporting enzyme cuvets, $\mathrm{v}_{\mathrm{E} 1}$ and $\mathrm{v}_{\mathrm{E} 2}$, respectively, are taken as the $\Delta \mathrm{Abs}_{340} /$ time values (typically the average of two runs) seen over the time window.

- The parameters, $\mathrm{Sel}_{E 1}$ and $\mathrm{Sel}_{E 2}$, were taken as the observed reporting enzyme enantioselectivies @ $40 \mathrm{mM}$ diol concentrations (see Table S2):

$$
\begin{aligned}
& S e l_{H L A D H=E 1}=\mathrm{v}_{\mathrm{R}} / \mathrm{v}_{\mathrm{S}}(\mathrm{HLADH}) @ 40 \mathrm{mM}=0.34 \\
& S e l_{T B A D H=E 2}=\mathrm{v}_{\mathrm{R}} / \mathrm{v}_{\mathrm{S}}(\mathrm{TBADH}) @ 40 \mathrm{mM}=8.4
\end{aligned}
$$

This approximation was chosen, based upon the following considerations. Control experiments (see Section IX, below) established that under ISES conditions, 1,2-propanediol concentrations in the aqueous layer were in the range of 2-100 mM, for active catalysts over the observed time window. As noted previously, the fact that $\mathrm{K}_{\mathrm{m}}$ 's do not vary widely suggested that fixing selectivity factors in this way, might, indeed be a reasonable approximation, and permit us to make useful estimations of $(R)$ and $(S)$-diol concentrations.

The enantiomeric excesses predicted from our double cuvet-ISES data, using this equation and these empirical selectivity factors, for Co(III)-salen complexes in the library, indexed by salen, are displayed in indigo in Table 1 of the manuscript. For comparison purposes, ee's determined for the same catalysts under standard 'Jacobsen conditions'? (neat propylene oxide, no co-solvent, 0.55 equiv. $\mathrm{H}_{2} \mathrm{O}, 3 \mathrm{~h}$ reaction time) are displayed below each prediction in black. 


\section{Estimation of Relative Rates for Catalysts Screened}

In order to rank catalysts in terms of relative rate, ISES rates, $\mathrm{v}_{\mathrm{E} 1}$ and $\mathrm{v}_{\mathrm{E} 2}$, over the 15 minute time window, were imported into the expression for [total diol $]_{\text {catalyst }} \mathrm{A} /[\text { total diol }]_{\text {catalyst B }}$ shown below (see Appendix for derivation). Note that the selectivity factors used here, $\mathrm{Sel}_{\mathrm{E} 1}$ and $\mathrm{Sel}_{\mathrm{E} 2}$, are the same as those used in the expression for enantioselectivity just discussed (Section VI). For comparison purposes, these relative rate rankings were compared with actual rate data obtained by observing diol formation by ${ }^{1} \mathrm{H}$ NMR (vide infra, Section IX). Since those empirical data were normalized to the rate of the catalyst derived from salen $\mathbf{3 b}$, the predicted relative rates were similarly normalized. A comparison of the predicted and observed rates is presented in Figure 2 of the manuscript.

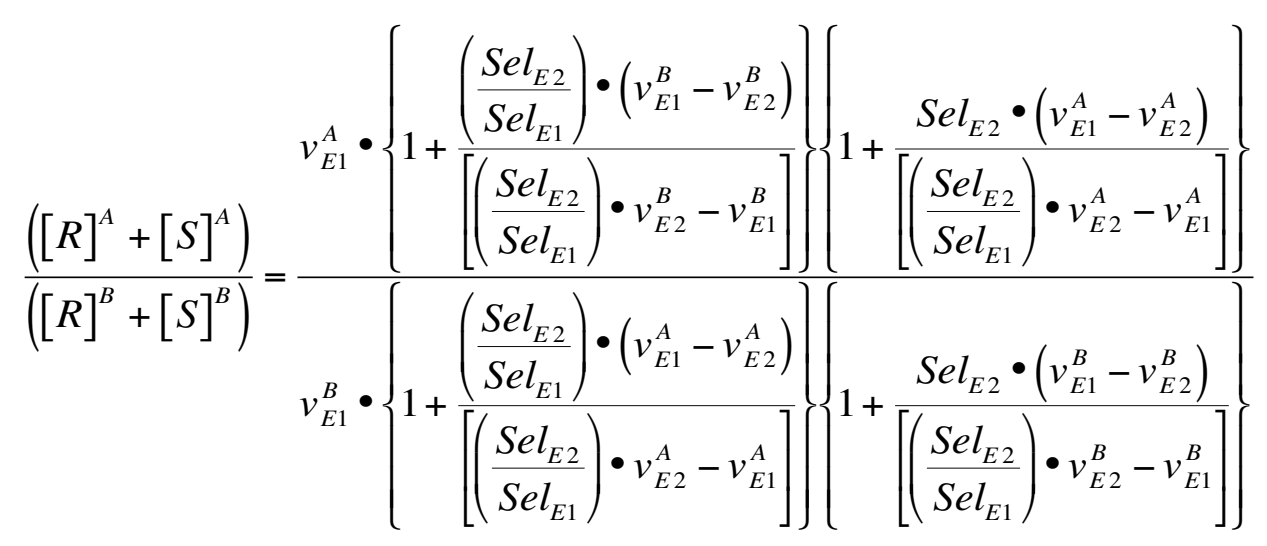




\section{Independent Measures of Enantioselectivity}

\section{A. Standard 'Jacobsen Conditions'}

Salens in the fully synthetic, 42 member focused library that led to catalysts with appreciable activity, as indicated by double cuvet-ISES, were also evaluated 'outside of the cuvet,' under conditions more typically employed in HKR reactions. Specifically, these Co(III)-salen catalysts were evaluated in the HKR of $( \pm)$-propylene oxide, under neat conditions, similar to those described by Jacobsen and coworkers. ${ }^{7}$ Reactions were arbitrarily stopped after $3 \mathrm{~h}$, to obtain the enantiopurity of the 1,2propanediol product at relatively early reaction times, for comparison with ISES predictions.

A typical procedure follows: To the Co(III)-salen catalyst derived from 7e $(5.4 \mathrm{mg}, 5.4 \mu \mathrm{mol}$, $0.25 \mathrm{~mol} \%)$ and ( \pm )-propylene oxide $(125 \mathrm{mg}, 2.15 \mathrm{mmol})$ at $0{ }^{\circ} \mathrm{C}$, was added water $(21 \mu \mathrm{L}, 1.18 \mathrm{mmol}$, 0.55 equiv.). The reaction mixture was securely capped, and allowed to warm to $\mathrm{rt}$ and stirred, most commonly for a total of $3 \mathrm{~h}$. Percent conversion at $3 \mathrm{~h}$ was evaluated by ${ }^{1} \mathrm{H}$ NMR of an aliquot (cooled to $0{ }^{\circ} \mathrm{C}$ for the transfer), in $\mathrm{CDCl}_{3}$. In most of these cases, the conversion was low enough after $3 \mathrm{~h}$, that the unreacted epoxide (bp $32{ }^{\circ} \mathrm{C}$ ) was of relatively low ee, and was therefore removed by evaporation under aspirator vacuum. When the conversion was less than $5 \%$ by NMR after $3 \mathrm{~h}$, reactions were continued for longer times.

Upon termination of the reaction, the diol product was isolated by Kugelrohr distillation $(\sim 120$ ${ }^{\circ} \mathrm{C}$-oven temp; 0.1 torr). To determine ee, the isolated diol was derivatized using $p$-bromobenzoyl imidazole (2.2 equiv.) in THF in the presence of $\mathrm{NaH}$ (4 equiv.) at rt for $5 \mathrm{~min}$. The bis $(p$ bromobenzoate) esters obtained following sequential washes with $1 \mathrm{~N} \mathrm{HCl}$ and saturated sodium bicarbonate, were of sufficient purity to be used directly for chiral HPLC analysis (Chiralcel OD, 99\% hexane/i-PrOH). A tabulation of observed ee's (chiral HPLC), percent conversions (NMR), isolated yields (Kugelrohr distallation) is presented in Table S4, below. Representative chiral HPLC traces are provided in Section XIII (vide infra). 
Table S4: HKR Under Standard 'Jacobsen Conditions' with Co(III)-Salen Catalyst 'Hits'

\begin{tabular}{|c|c|c|c|c|}
\hline Salen & $\begin{array}{c}\text { Loading } \\
(\operatorname{mol} \%)\end{array}$ & $\begin{array}{l}\text { NMR } \\
\text { conv. } \\
\text { (time) }\end{array}$ & $\%$ Yield (time) & $\%$ ee (HPLC) \\
\hline $1 \mathbf{a}$ & 0.25 & $12(3 \mathrm{~h})$ & $14(3 \mathrm{~h})$ & $+72(S)$ \\
\hline $\mathbf{2 a}$ & 0.25 & $17(3 \mathrm{~h})$ & $24(5 \mathrm{~h})$ & $-93(R)$ \\
\hline $\mathbf{3 a}$ & 0.25 & $46(3 \mathrm{~h})$ & $39(3 \mathrm{~h})$ & $+55(S)$ \\
\hline $4 \mathbf{a}$ & 0.25 & $35(3 \mathrm{~h})$ & $26(3 \mathrm{~h})$ & $+76(S)$ \\
\hline $5 \mathbf{a}$ & 0.25 & $36(3 \mathrm{~h})$ & $30(3 \mathrm{~h})$ & $+66(S)$ \\
\hline $6 \mathbf{a}^{*}$ & 0.25 & $12(3 \mathrm{~h})$ & $16(3 \mathrm{~h})$ & $+69(S)$ \\
\hline $7 a^{*}$ & 0.25 & $7(3 \mathrm{~h})$ & $6(3 \mathrm{~h})$ & $-64(R)$ \\
\hline $\mathbf{1 b}$ & 0.25 & n.d. & $35(3 \mathrm{~h})$ & $+75(S)$ \\
\hline $2 \mathbf{b}$ & 0.25 & $26(3 \mathrm{~h})$ & $29(3 \mathrm{~h})$ & $-75(R)$ \\
\hline $\mathbf{3 b}$ & 0.25 & n.d. & $17(3 \mathrm{~h})$ & $+57(S)$ \\
\hline $4 b$ & 0.25 & $50(3 \mathrm{~h})$ & $32(3 \mathrm{~h})$ & $+59(S)$ \\
\hline $5 \mathbf{b}$ & 0.25 & $45(3 \mathrm{~h})$ & $36(3 \mathrm{~h})$ & $+68(S)$ \\
\hline $6 b^{*}$ & 0.25 & $11(3 \mathrm{~h})$ & $12(3 \mathrm{~h})$ & $+42(S)$ \\
\hline $7 b^{*}$ & 0.25 & $9(3 \mathrm{~h})$ & $13(3 \mathrm{~h})$ & $-40(R)$ \\
\hline $3 c$ & 0.25 & none obs & & \\
\hline $7 c$ & 0.25 & none obs & & \\
\hline $1 d$ & 0.25 & n.d. & $21(1 \mathrm{~h}), 33(3 \mathrm{~h})$ & $+81 @ 1 \mathrm{~h},+67 @ 3 \mathrm{~h}(S)$ \\
\hline $2 d$ & 0.25 & $19(22 \mathrm{~h})$ & $15(22 \mathrm{~h})$ & $-54(R)$ \\
\hline $3 d$ & 0.25 & $10(3 \mathrm{~h})$ & $7(3 \mathrm{~h})$ & $-30(R)$ \\
\hline $5 d$ & 0.25 & $10(3 \mathrm{~h})$ & $9(3 \mathrm{~h})$ & $+11(S)$ \\
\hline $1 e$ & 0.25 & n.d. & $36(3 \mathrm{~h})$ & $+28(S)$ \\
\hline $2 \mathrm{e}$ & 0.25 & $26(3 \mathrm{~h})$ & $18(3 \mathrm{~h})$ & $-41(R)$ \\
\hline $4 e$ & 0.25 & $6(24 \mathrm{~h})$ & $6(24 h)$ & $+14(S)$ \\
\hline $5 e$ & 0.25 & $3(3 \mathrm{~h})$ & $3(3 \mathrm{~h})$ & $-5(R)$ \\
\hline $7 e$ & 0.25 & $33(3 \mathrm{~h})$ & $12(3 \mathrm{~h})$ & $+81(S)$ \\
\hline $7 e^{*}$ & 0.05 & $10(3 \mathrm{~h})$ & $16(3 \mathrm{~h})$ & $+83(S)$ \\
\hline $4 f$ & 0.25 & $24(21 \mathrm{~h})$ & $23(21 \mathrm{~h})$ & $+51(S)$ \\
\hline $2 \mathrm{~g}$ & 0.25 & n.d. & $1(3 \mathrm{~h}), 4(20 \mathrm{~h})$ & -71@3h,-65@20h(R) \\
\hline
\end{tabular}

n.d. = not determined; *Indicates that the Co(III)-3,5-dinitrobenzoate catalyst was used. All other entries are for Co(III)-salen-acetate catalysts. 


\section{B. Catalyst Performance in the ISES Cuvet}

For several catalysts, control experiments were run to assess whether running the HKR under biphasic conditions influences the enantioselectivity of theses Co(III)-salen HKR catalysts. In such cases, parallel duplicate 'double-cuvet' runs were chosen (i.e. two HLADH cuvets and two TBADH cuvets for a single catalyst). Following the ISES runs, the contents of all four reporting cuvets were merged. Unreacted epoxide was rapidly removed under Ar stream. Following dilution with $\mathrm{CH}_{2} \mathrm{Cl}_{2}$ ( $\sim 50 \mathrm{~mL})$, sodium sulfate was added to adsorb remaining water. The resulting organic solution was filtered and the filter cake washed with $\mathrm{CH}_{2} \mathrm{Cl}_{2}(2 \times 20 \mathrm{~mL})$. Following evaporation of the solvent, the crude diols were derivatized directly with $p$-bromobenzoyl imidazole to yield the 1,2-propanediol bis $(p$ bromobenzoate) esters, which were then purified by silica gel chromatography to estimate diol yields. Enantiomeric excesses were determined using chiral HPLC, as before. As can be seen from Table S5, there is no appreciable erosion of ee seen in running these HKR experiments under biphasic conditions. Indeed, the ee's seen from diol isolated directly out of the cuvet generally appear slightly higher than those obtained under 'Jacobsen conditions,' probably reflecting the shorter reaction times employed in the ISES experiment.

Table S5: Biphasic vs. 'Jacobsen Conditions'

\begin{tabular}{|c|c|c|c|c|}
\hline $\begin{array}{c}\text { Co(III)-salen } \\
\text { (indexed by } \\
\text { salen) }\end{array}$ & $\begin{array}{c}\text { \% Yield } \\
\text { (isolated) }\end{array}$ & $\begin{array}{c}\% \text { ee }_{\text {measured }} \\
\text { biphasic conds. }\end{array}$ & $\begin{array}{c}\% \text { ee }_{\text {measured }} \\
\text { neat 'Jacobsen } \\
\text { conditions' }\end{array}$ & $\begin{array}{c}\% \text { ee }_{\text {predicted }} \\
\text { double cuvet- } \\
\text { ISES }\end{array}$ \\
\hline $\mathbf{1 a}$ & 1 & 87 & 72 & 56 \\
\hline $\mathbf{4 a}$ & 1.6 & 82 & 76 & 77 \\
\hline $\mathbf{7 a}$ & $<1$ & -55 & -64 & -33 \\
\hline $\mathbf{7 e}$ & 0.4 & 85 & 81 & 87 \\
\hline
\end{tabular}

* denotes that the Co(III)-salen-3,5-dinitrobenzoate catalyst was used in this case. All other entries refer to runs with $\mathrm{Co}(\mathrm{III})$-salen-acetate catalysts. 


\section{Independent Measurement of Relative Rates of Catalysts}

The concentration of 1,2-propanediol in the aqueous layer was estimated under typical ISES conditions using ${ }^{1} \mathrm{H}$ NMR spectroscopy. The bilayer was prepared as usual for double cuvet-ISES (Section V), but for the substitution of $\mathrm{D}_{2} \mathrm{O}$ for $\mathrm{H}_{2} \mathrm{O}$ in the buffer, and the elimination of the dehydrogenase enzymes. After $15 \mathrm{~min}$, the aqueous layer $(300 \mu \mathrm{L}$ of $500 \mu \mathrm{L}$ total) was withdrawn and mixed with a fresh solution of imidazole of known concentration (also $300 \mu \mathrm{L}$ in $\mathrm{D}_{2} \mathrm{O}$ ), as an internal concentration standard. Accumulation of the ${ }^{1} \mathrm{H}$ NMR spectrum, followed by integration and quantitation, provided an experimental measure of [total diol] in the aqueous reporting layer at $t$ $=15$ minutes for the catalyst under investigation. The observed diol concentrations for ten catalysts examined in this way are collected in Table S4, below. These have been normalized to the value for the catalyst derived from salen $\mathbf{3 b}$ (arbitrarily assigned a rate of 1.0).

Table S6: Co(III)-salen-Mediated HKR: [total 1,2-Propanediol] by NMR (aqueous ISES layer)

\begin{tabular}{|l|l|l|l|l|l|l|l|l|l|l|}
\hline Salen & $\mathbf{1 a}$ & $\mathbf{2 a}$ & $\mathbf{2 b}$ & $\mathbf{1 d}$ & $\mathbf{3 b}$ & $\mathbf{4 b}$ & $\mathbf{7 e}$ & $\mathbf{5 a}$ & $\mathbf{5 b}$ & $\mathbf{5 d}$ \\
\hline $\begin{array}{l}\text { [diol] } \\
(\mathrm{mM}) @ \\
15 \mathrm{~min}\end{array}$ & 19 & 27.5 & 22 & 91 & 96 & 98 & 37 & 52.5 & 75.5 & 54 \\
\hline Norm. & 0.20 & 0.29 & 0.23 & 0.95 & 1.0 & 1.02 & 0.39 & 0.55 & 0.79 & 0.56 \\
\hline
\end{tabular}

\section{References}

1. Snider, B. B.; Lin, H. Synth. Commun. 1998, 1913-1922.

2. Y. Hsiao, L. S. Hegedus; J. Org. Chem. 1997, 62, 3586-3591.

3. Kihlberg, T.; Karimi, F.; Langstrom, B. J. Org. Chem. 2002, 67, 3687-3692.

4. Hsu, F-L.; Hamada, A.; Booheer, M. E.; Fuder, H.; Patil, P. N.; Miller, D. D. J. Med. Chem., 1980, 23, 1232-1235.

5. Brunner, H.; Hankofer, P.; Holzinger, U.; Treittinger, B.; Schoenenberger, H.; Eur. J. Med. Chem. 1990, 25, 45-44.

6. Praly, J-P.; Bonnevie, C.; Haug, P.; Descotes, G. Tetrahedron 1996, 52, 9057-9068.

7. Schaus, S. E.; Brandes, B. D.; Larrow, J. F.; Tokunaga, M.; Hansen, K. B.; Gould, A. E.; Furrow, M. E.; Jacobsen, E. N. J. Am. Chem. Soc. 2002, 124, 1307-1315.

8. Ottolina, G.; Carrea, G.; Riva, S. Bückmann, A. F. Enzyme Microb. Technol. 1990, 12, 596-602.

9. Dilmaghaninan, S.; Stead, C. V.; Ansell, R. J.; Lowe, C. R. Enzyme Microb. Technol. 1997, 20, 165-173.

10. (a) Berkowitz, D. B.; Maiti, G. Org. Lett. 2004, 6, 2661-2664; (b) Berkowitz, D. B.; Shen, W.; Maiti, G. Tetrahedron: Asymmetry 2004, 15, 2845-2851; (c) Berkowitz, D. B.; Bose, M.; Choi, S. Angew. Chem., Int. Ed. 2002, 41, 1603-1607. 


\section{Appendix: Derivation of Expressions for Enantioselectivity and Relative Rate}

\section{Potential Application of 'Double Cuvet-ISES' (Two Reporting Enzymes) to Rank Two Catalysts in Terms of Relative Enantioselectivities and Relative Rates}

Let us say that we are screening two chiral catalysts A and B, capable of producing a chiral product. a) Assume that we have two different enzymes E1 and E2 each of which takes both $(R)$-product and $(S)$-product as substrate, but with different relative velocities:

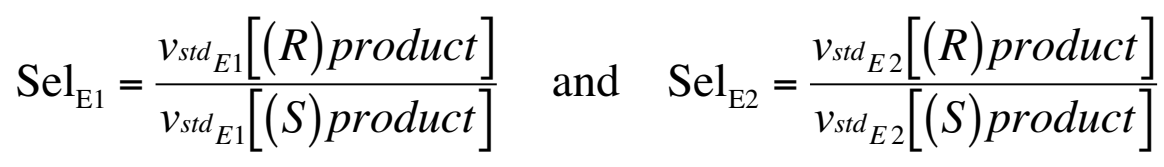

Note: These selectivity parameters are experimentally determined relative velocities at a chosen 'standard' substrate concentration appropriate to the ISES experiment.

b) We assume further that $S e l_{\mathrm{E} 1}$ and $S e l_{\mathrm{E} 2}$ can be approximated as fixed under the conditions of the experiment.

Notes: This selectivity factor will tend toward $\left[\left(\mathrm{k}_{\mathrm{cat}} / \mathrm{K}_{\mathrm{m}}\right)_{R} /\left(\mathrm{k}_{\mathrm{cat}} / \mathrm{K}_{\mathrm{m}}\right)_{S}\right]$ for low substrate concentrations $\left([S]<<\mathrm{K}_{\mathrm{m}}\right)$. It will tend toward $\left[\left(\mathrm{k}_{\mathrm{cat}-R} / \mathrm{k}_{\mathrm{cat}-S}\right]\right.$ for very high substrate concentrations $\left([S]>>\mathrm{K}_{\mathrm{m}}\right)$. And it will be some hybrid for intermediate concentrations, which turns out to be the most common situation seen empirically here (see Sections IV and IX). Since we expect [S] to increase in the time window of our screen, we have chosen to record an average velocity $(\Delta \mathrm{OD} / \mathrm{t})$ for each reporting enzyme in that window. For this reason, we have chosen as a selectivity factor the measured relative $(R):(S)$ velocities observed for each enzyme in the approximate diol concentration range in which we are operating.

c) Let $\mathrm{x}=$ the number of $(R)$-product units of both enzyme E1 and enzyme E2 used in their respective cuvets/wells

Then, assuming the rates of enantiomer turnover are additive, within some reasonable experimental uncertainty, we obtain:

$$
\begin{aligned}
& v_{E 1}=x[R]+\frac{x}{S e l_{E 1}}[S] \\
& v_{E 2}=x[R]+\frac{x}{S e l_{E 2}}[S]
\end{aligned}
$$

Note: Again, these rates are approximate. Enzymatic rates will be most cleanly first order in substrate at low diol concentrations (i.e. at early times) with pseudo-second order rate constant $\mathrm{k}_{\mathrm{cat}} / \mathrm{K}_{\mathrm{m}}$. The fact that the actual $\left(\mathrm{k}_{\text {cat }} / \mathrm{K}_{\mathrm{m}}\right)_{R} /\left(\mathrm{k}_{\mathrm{cat}} / \mathrm{K}_{\mathrm{m}}\right)_{S}$ values of the two reporting enzymes used here do not differ greatly from the relative $(R):(S)$ velocities seen at higher concentrations (see Section III) suggests that the use of these fixed selectivity factors is a reasonable approximation for these double cuvet-ISES experiments. The rates of turnover of the individual enantiomers are treated as being additive (implying 'independent'), in the interest of simplifying the expression. Of course, the simplified expression obtained then must be evaluated empirically to see if, in making these approximations, the resulting mathematical model allows for reasonable ISES predictions. This has been done for the HLADH/TBADH reporting enzymes here, and is presented in detail in Sections VI and VII, above. 
In other words:

$$
\begin{aligned}
& v_{E 1}=x\left\{[R]+\frac{[S]}{S e l_{E 1}}\right\} \\
& v_{E 2}=x\left\{[R]+\frac{[S]}{S e l_{E 2}}\right\}
\end{aligned}
$$

So:

$$
\frac{\left(v_{E 1}-v_{E 2}\right)}{\left[v_{E 1}-\left(\frac{S e l_{E 2}}{S e l_{E 2}}\right) \cdot v_{E 2}\right]}=\frac{x[S] \cdot\left(\frac{1}{S e l_{E 1}}-\frac{1}{S e l_{E 2}}\right)}{x[R] \cdot\left(1-\frac{S e l_{E 2}}{S e l_{E 1}}\right)}=\frac{[S]}{[R]}\left(-\frac{1}{S e l_{E 2}}\right)
$$

Therefore, the enantiomeric ratio is given by:

$\frac{[R]}{[S]}=\frac{\left[\left(\frac{S e l_{E 2}}{S e l_{E 1}}\right) \cdot v_{E 2}-v_{E 1}\right]}{S e l_{E 2} \bullet\left(v_{E 1}-v_{E 2}\right)}$

This equation will hold for catalysts $\mathrm{A}$ and $\mathrm{B}$, individually. It then allows us to compare the relative amounts of $(R)$-product formed in the $\mathrm{A}$ and $\mathrm{B}$ cuvets.

Substituting back into equation (3), for catalysts A and B, gives:

$$
\begin{aligned}
& v_{E 1}^{A}=X \cdot\left\{[R]^{A}+\frac{S e l_{E 2} \bullet\left(v_{E 1}^{A}-v_{E 2}^{A}\right) \bullet[R]^{A}}{S e l_{E 1} \bullet\left[\left(\frac{S e l_{E 2}}{S e l_{E 1}}\right) \cdot v_{E 2}^{A}-v_{E 1}^{A}\right]}\right\} \\
& v_{E 1}^{B}=X \bullet\left\{[R]^{B}+\frac{\operatorname{Sel}_{E 2} \bullet\left(v_{E 1}^{B}-v_{E 2}^{B}\right) \bullet[R]^{B}}{\operatorname{Sel}_{E 1} \bullet\left[\left(\frac{\operatorname{Sel}_{E 2}}{S e l_{E 1}}\right) \cdot v_{E 2}^{B}-v_{E 1}^{B}\right]}\right\}
\end{aligned}
$$


Dividing Equation (7) by (8), gives:

$$
\frac{v_{E 1}^{A}}{v_{E 1}^{B}}=\frac{[R]^{A}\left\{1+\frac{\left(\frac{S e l_{E 2}}{S e l_{E 1}}\right) \cdot\left(v_{E 1}^{A}-v_{E 2}^{A}\right)}{\left[\left(\frac{S e l_{E 2}}{S e l_{E 1}}\right) \cdot v_{E 2}^{A}-v_{E 1}^{A}\right]}\right\}}{[R]^{B}\left\{1+\frac{\left(\frac{S e l_{E 2}}{\operatorname{Sel} l_{E 1}}\right) \cdot\left(v_{E 1}^{B}-v_{E 2}^{B}\right)}{\left[\left(\frac{S e l_{E 2}}{S e l_{E 1}}\right) \cdot v_{E 2}^{B}-v_{E 1}^{B}\right]}\right\}}
$$

therefore:

$$
[R]_{r e l}=\frac{[R]^{A}}{[R]^{B}}=\frac{v_{E 1}^{A}\left\{1+\frac{\left(\frac{S e l_{E 2}}{S e l_{E 1}}\right) \cdot\left(v_{E 1}^{B}-v_{E 2}^{B}\right)}{\left[\left(\frac{S e l_{E 2}}{S e l_{E 1}}\right) \cdot v_{E 2}^{B}-v_{E 1}^{B}\right]}\right\}}{v_{E 1}^{B}\left\{1+\frac{\left(\frac{S e l_{E 2}}{\operatorname{Sel} l_{E 1}}\right) \cdot\left(v_{E 1}^{A}-v_{E 2}^{A}\right)}{\left[\left(\frac{S e l_{E 2}}{S e l_{E 1}}\right) \cdot v_{E 2}^{A}-v_{E 1}^{A}\right]}\right\}}
$$

Now, note that we can derive an expression for the (total) relative rates for catalysts A and B:

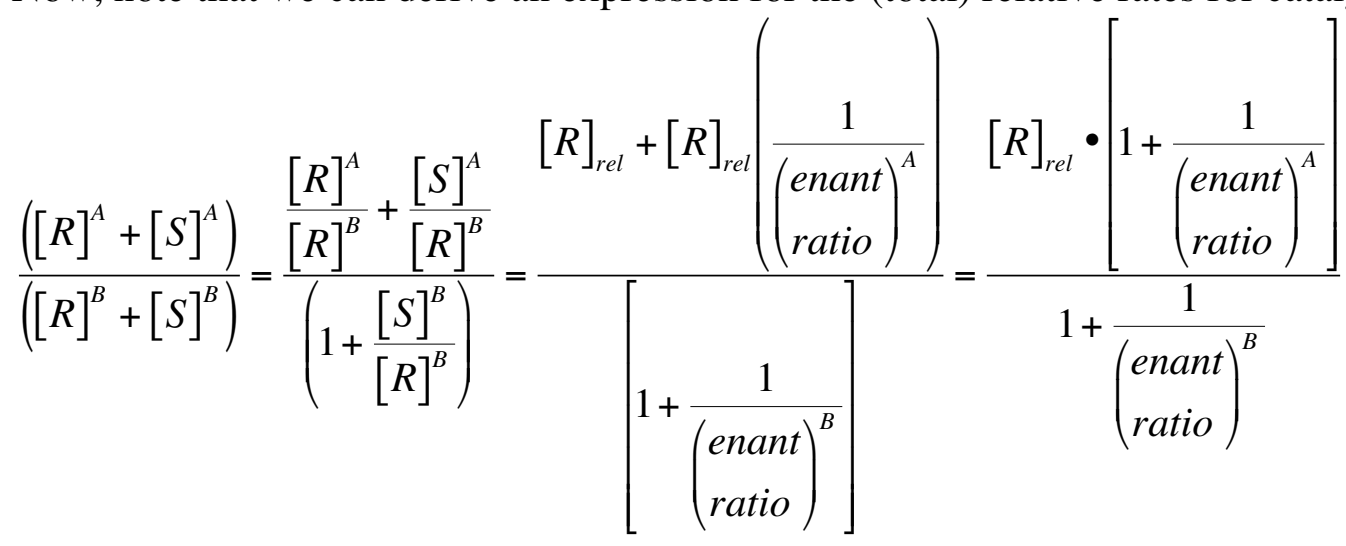

where: $\frac{[S]^{A}}{[R]^{A}} \cdot \frac{[R]^{A}}{[R]^{B}}=\frac{[S]^{A}}{[R]^{B}}$ 
Substituting from (6) and (10), we obtain:

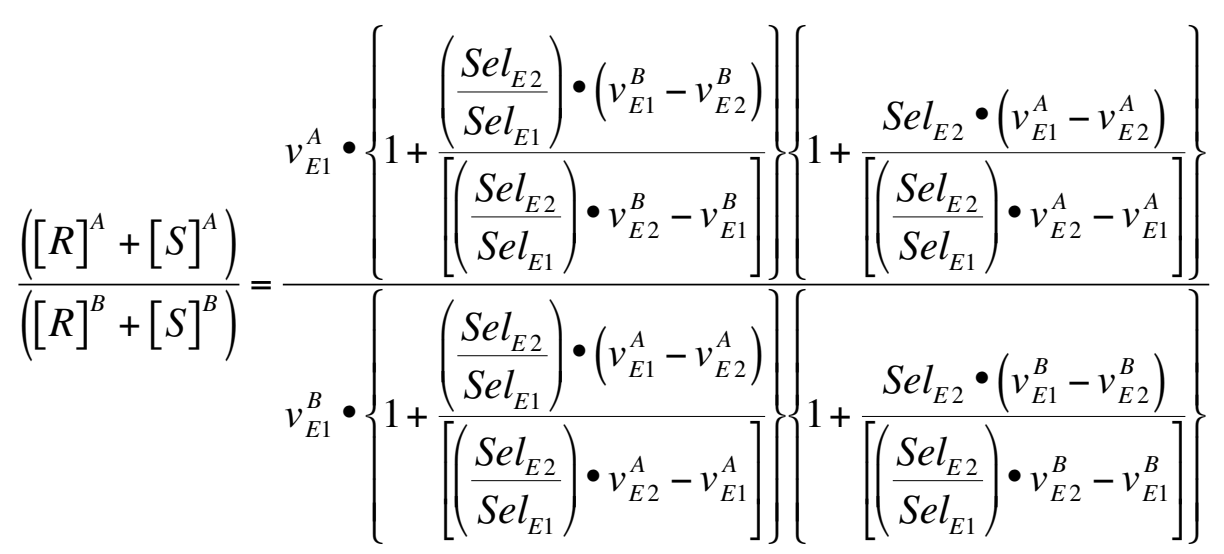


XII. ${ }^{1} \mathrm{H}$ NMR Spectra of Individual 'Salens' in the $7 \times 7$ Array

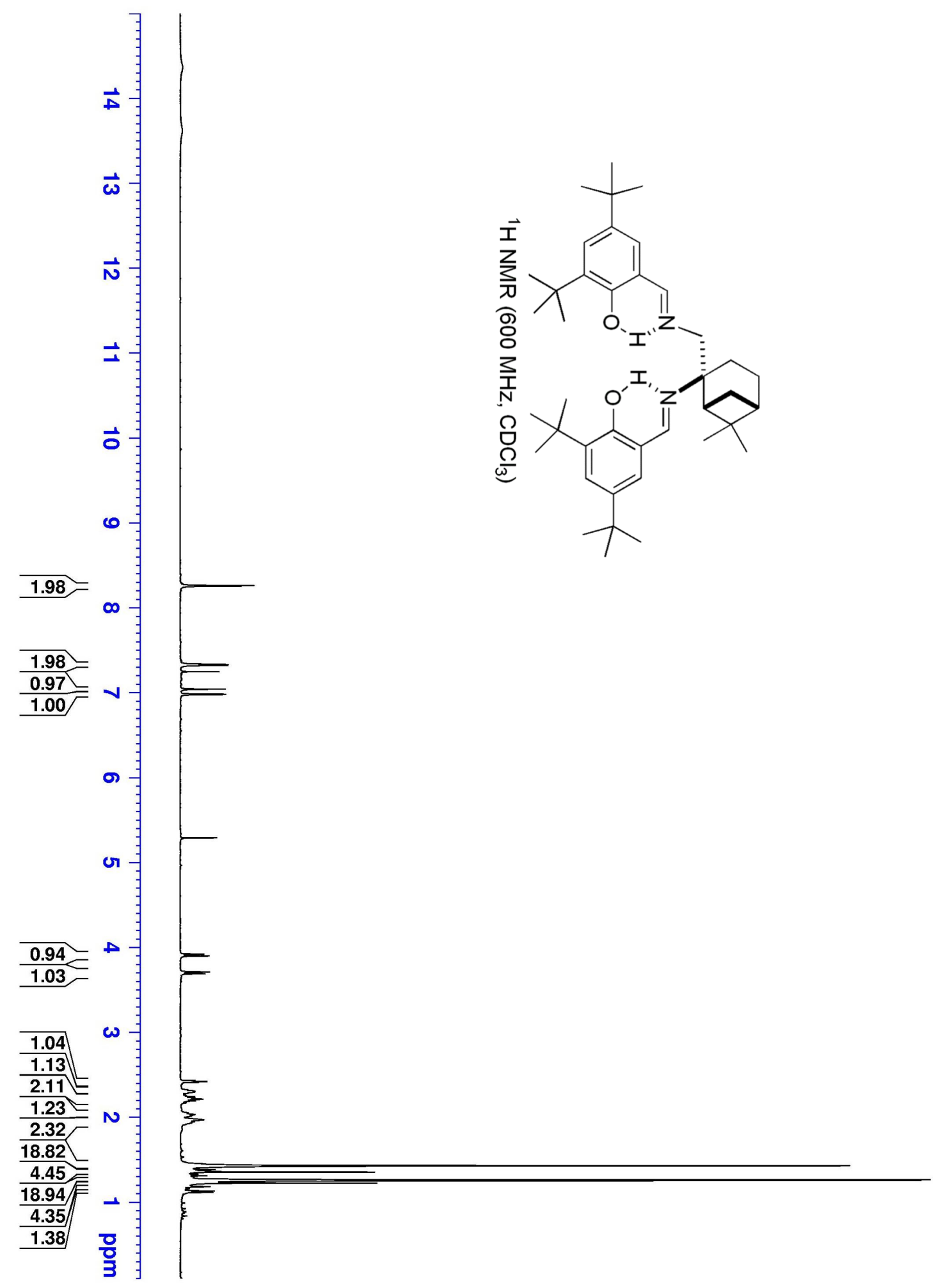




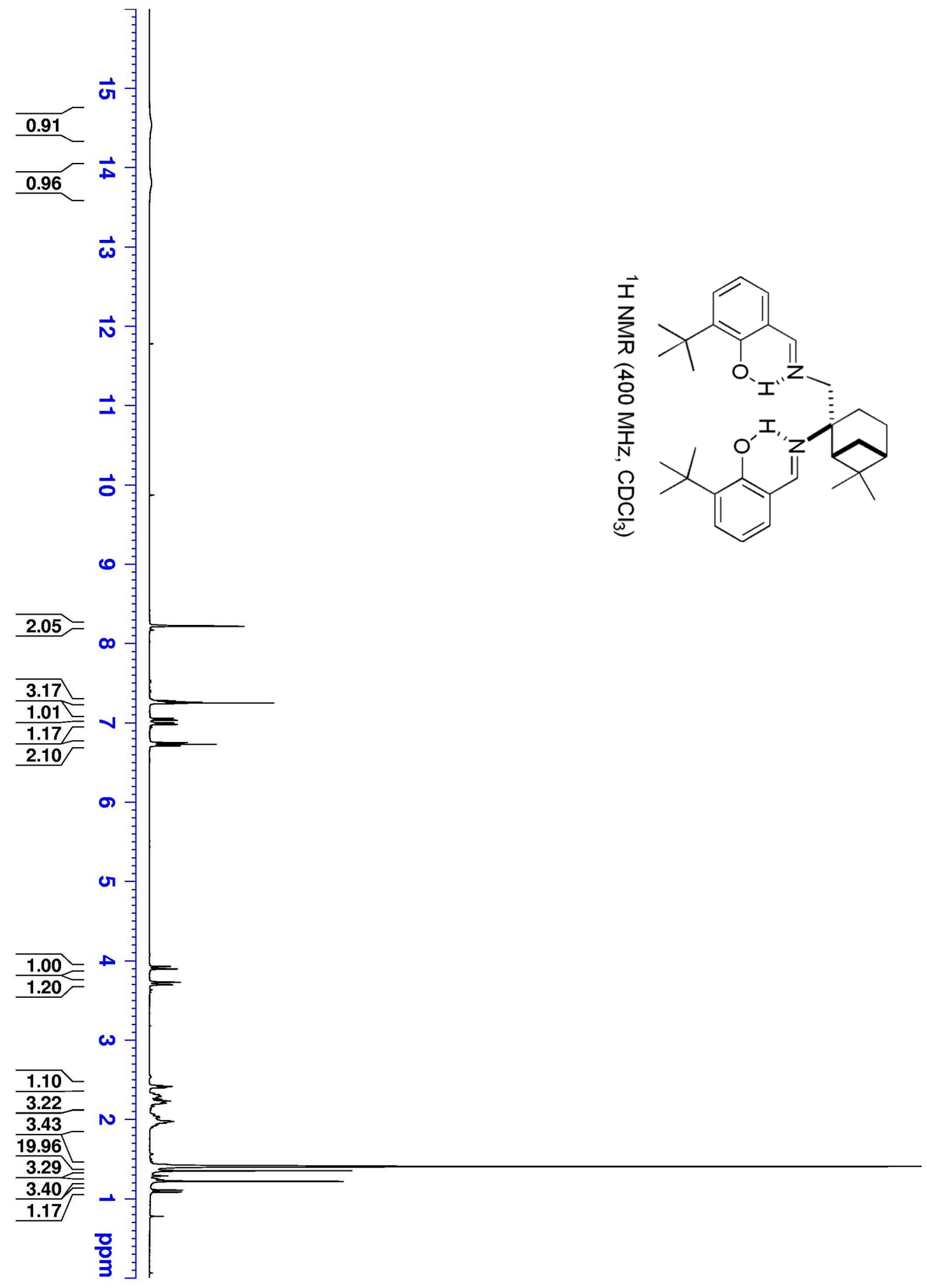




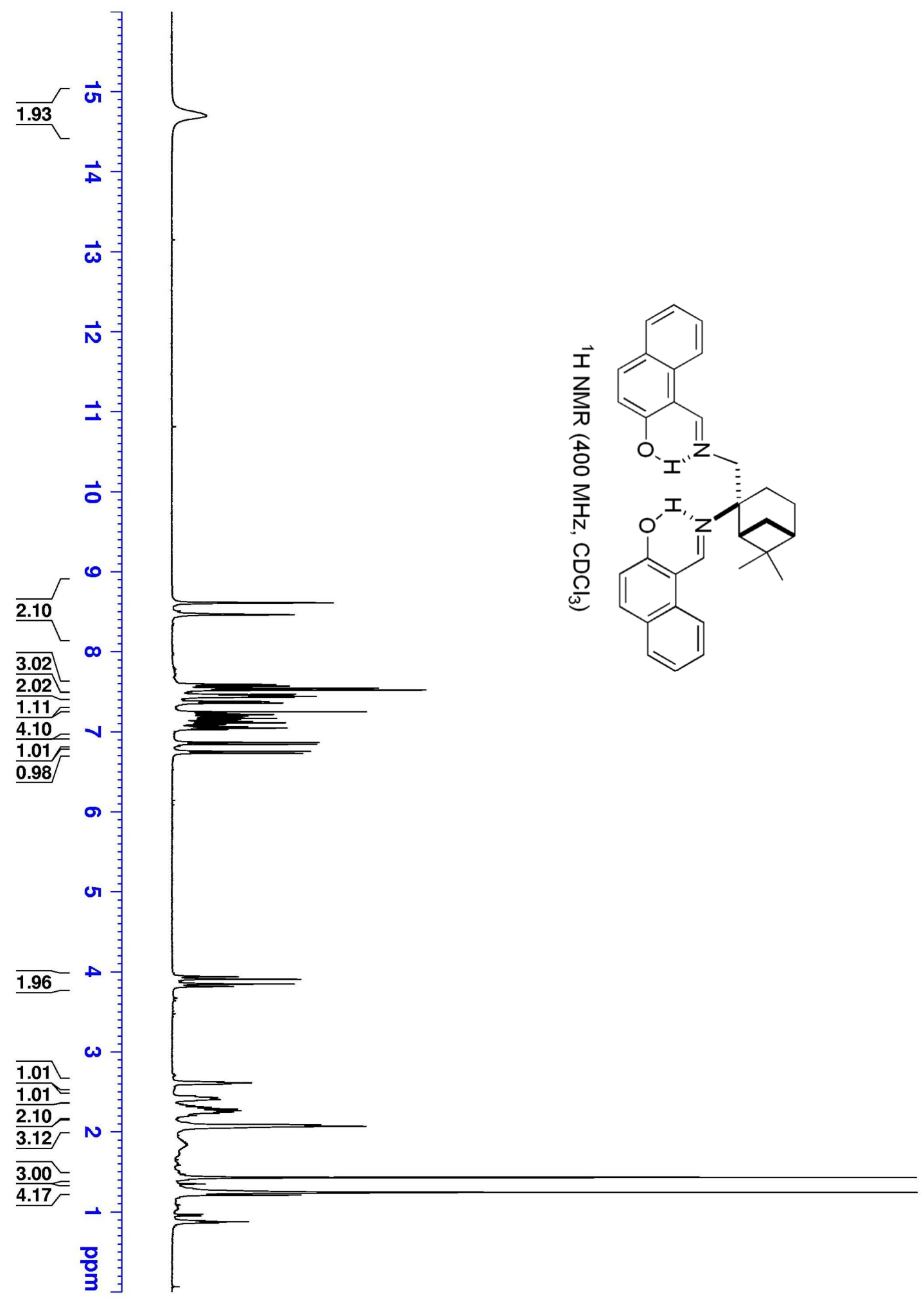




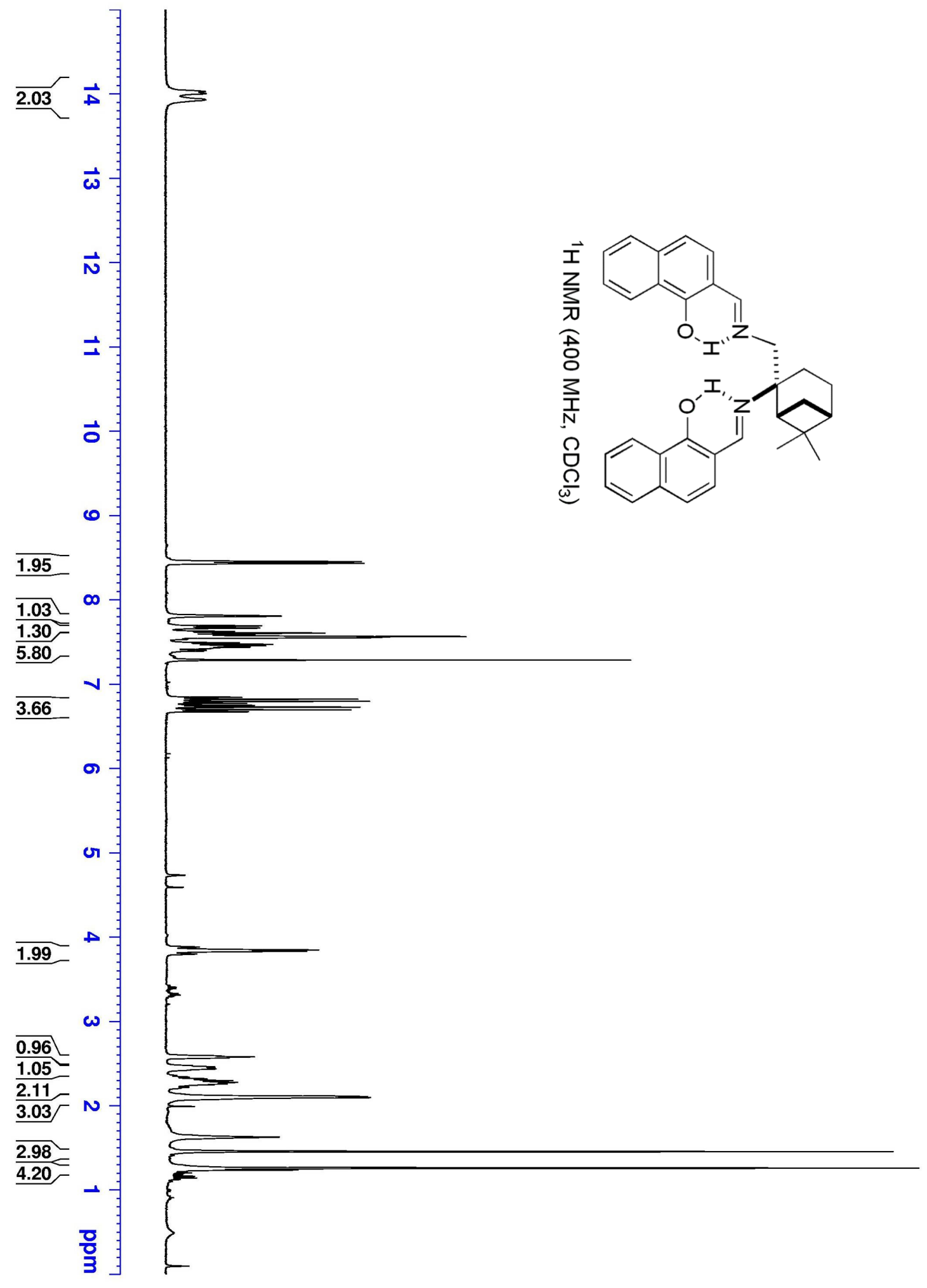




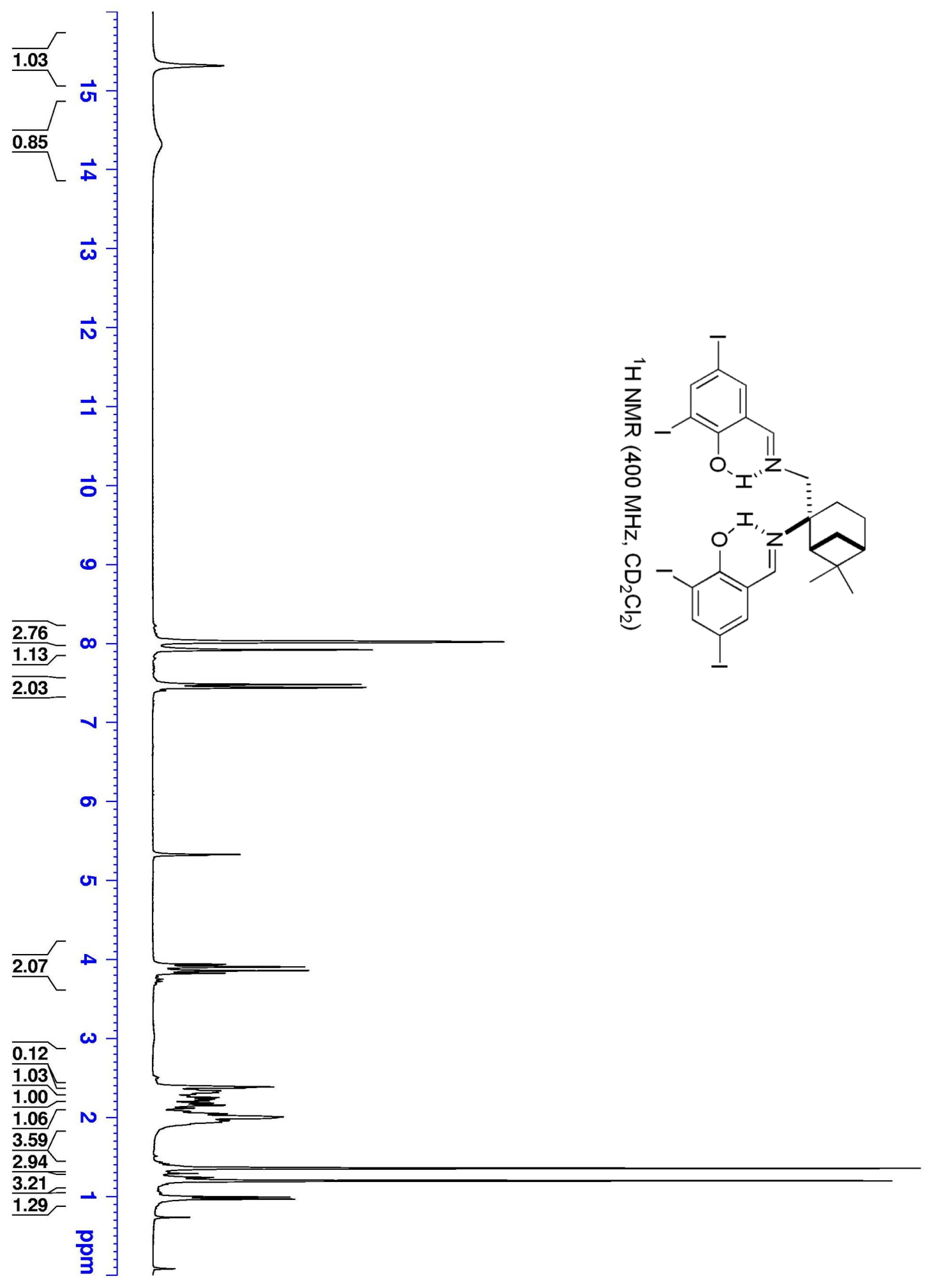




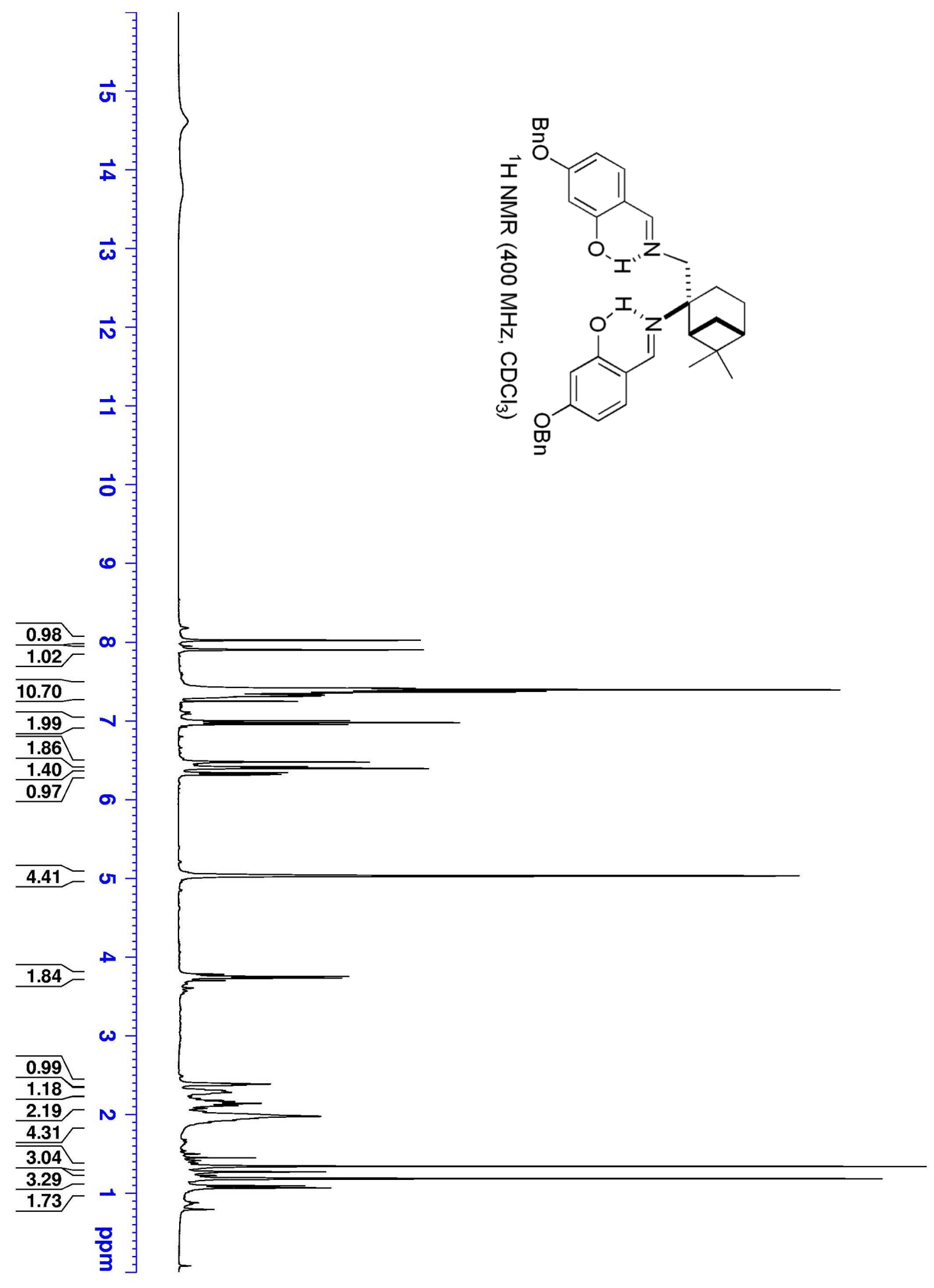




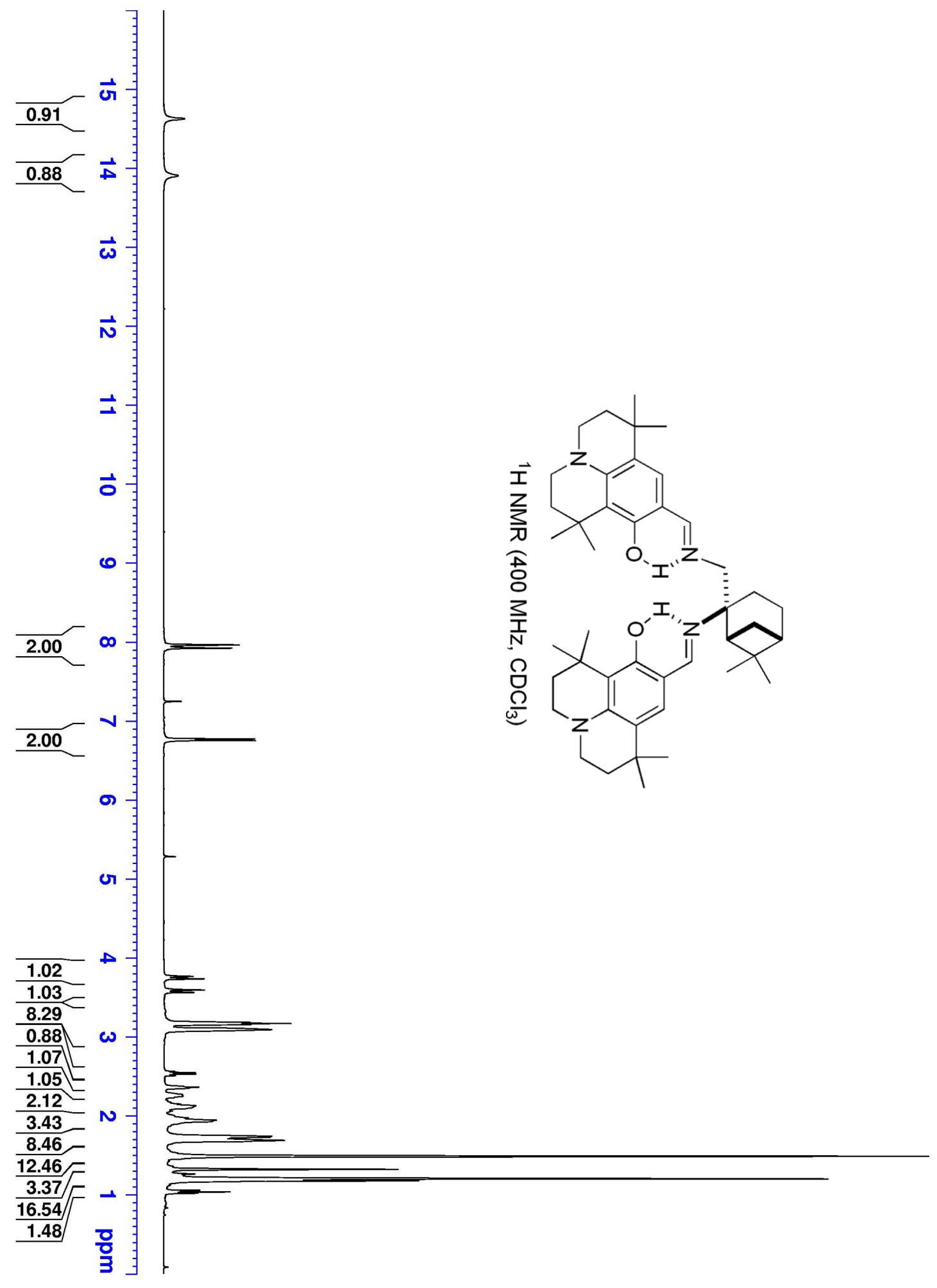




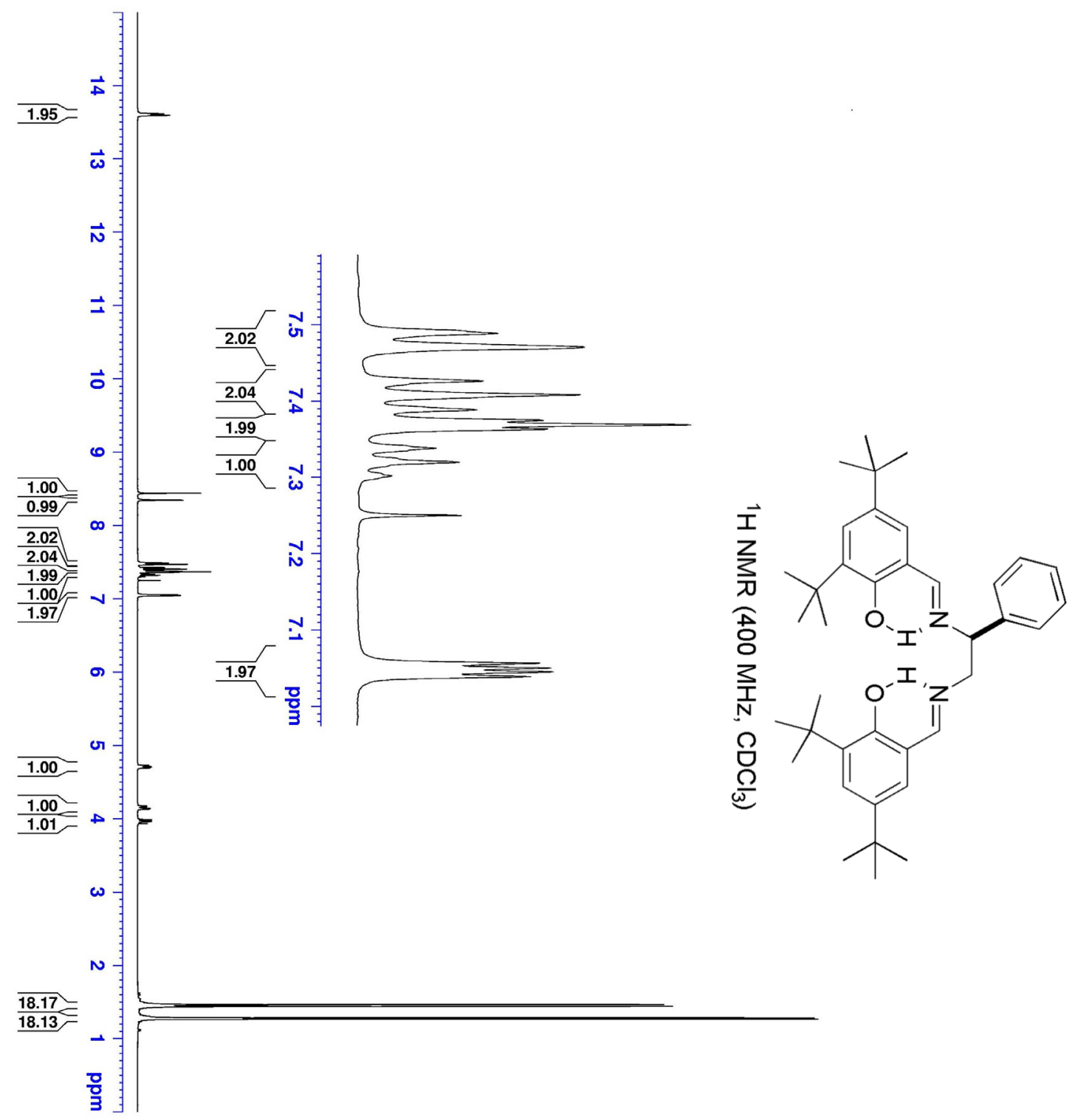




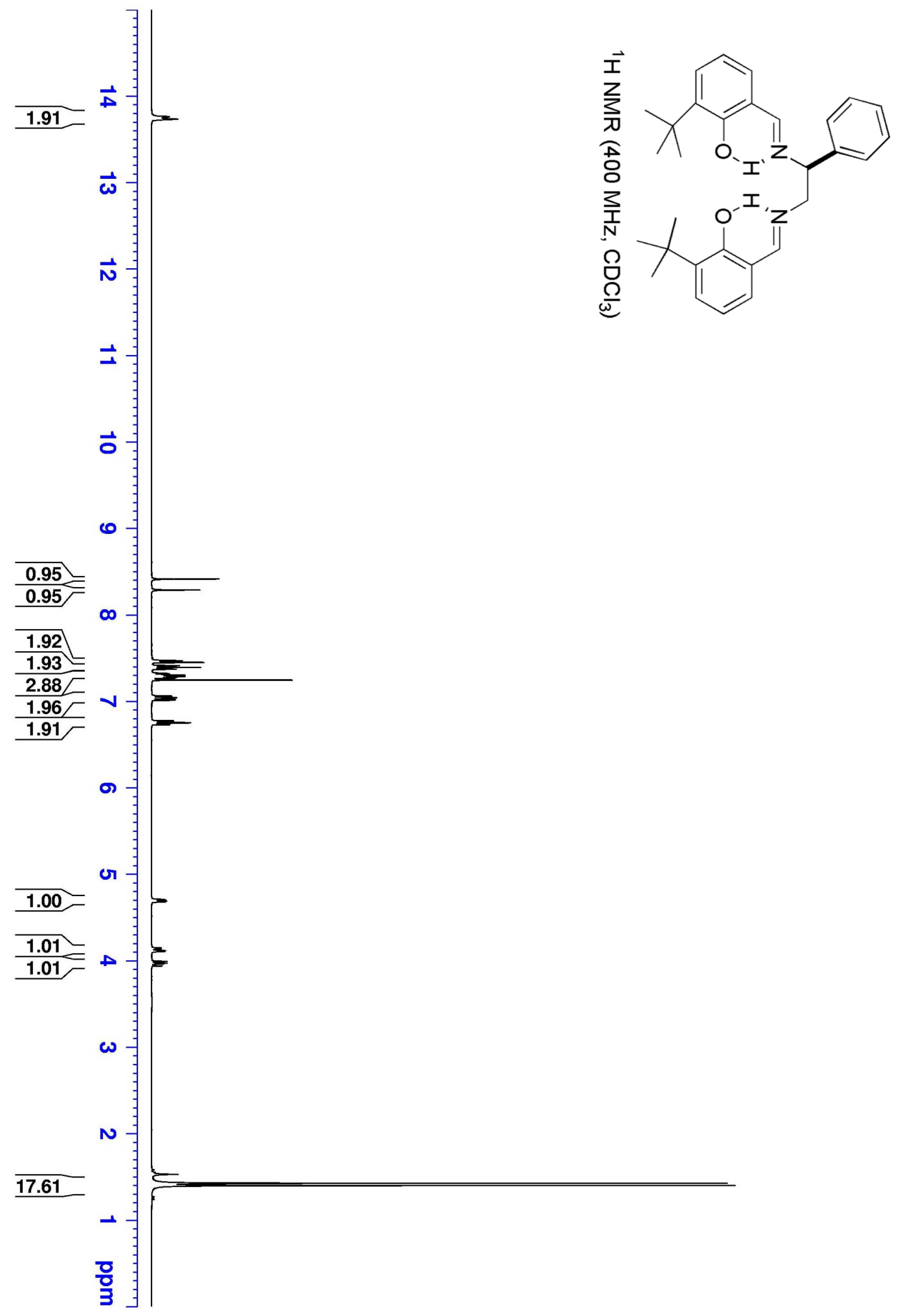




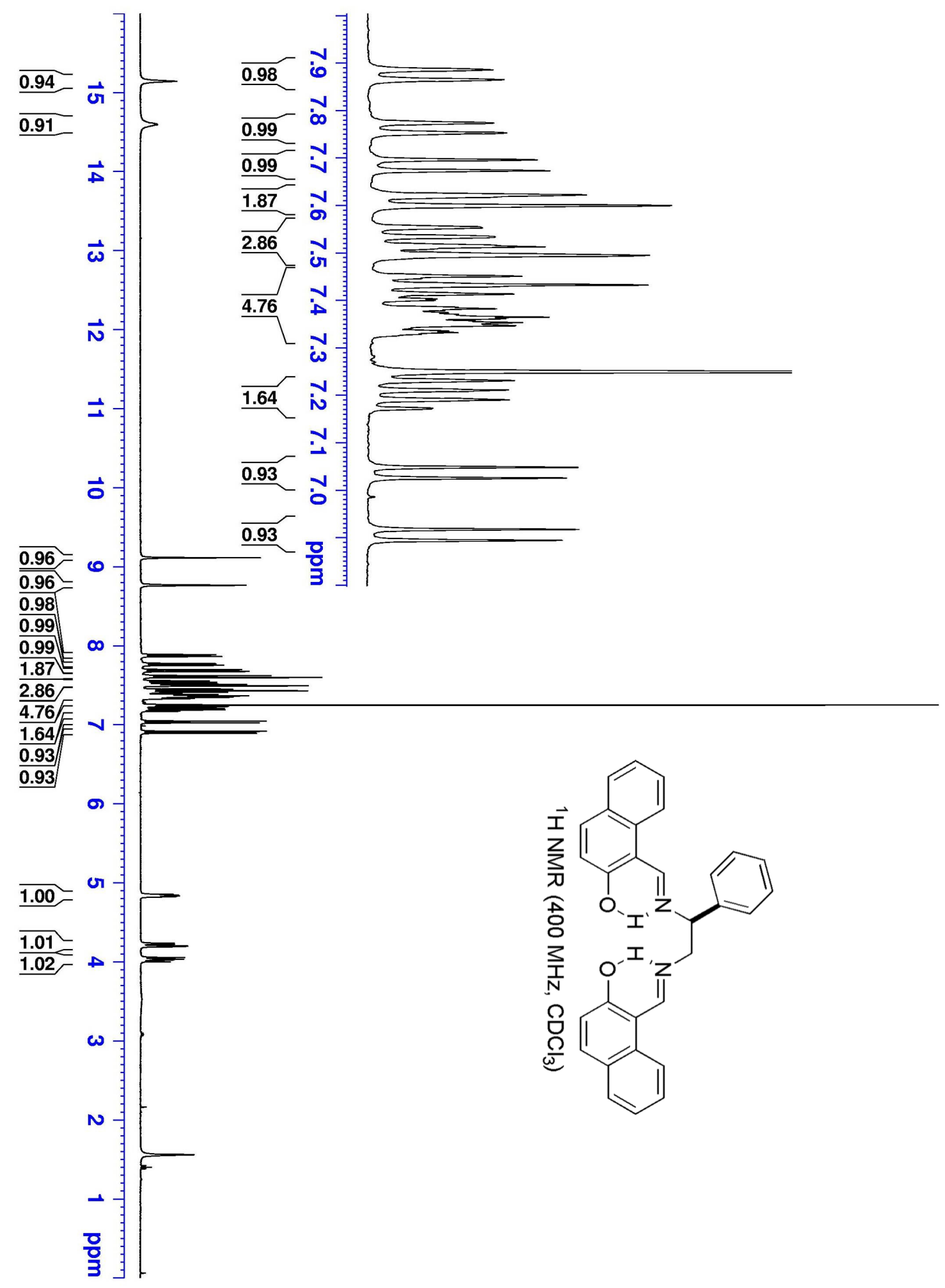




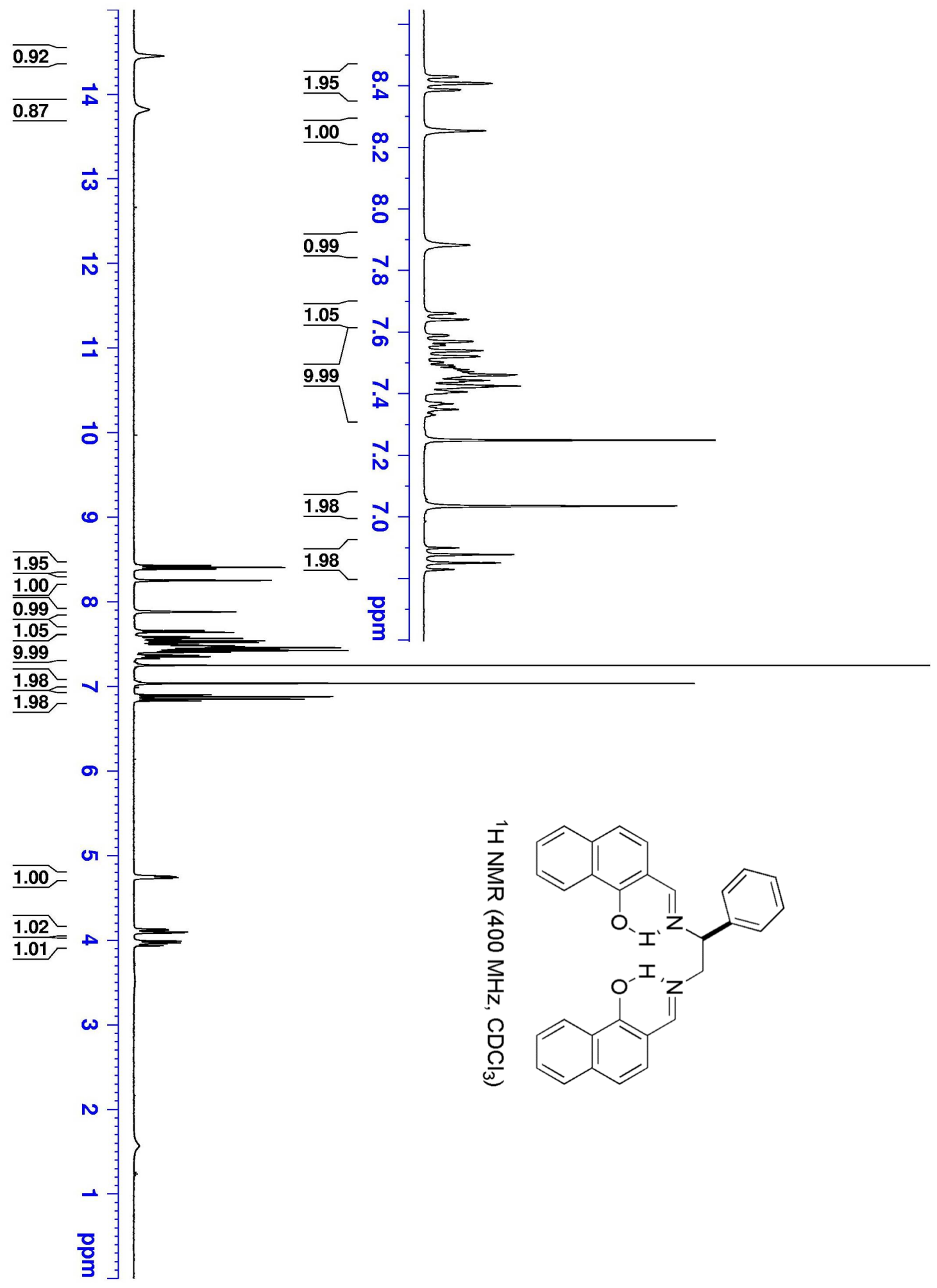




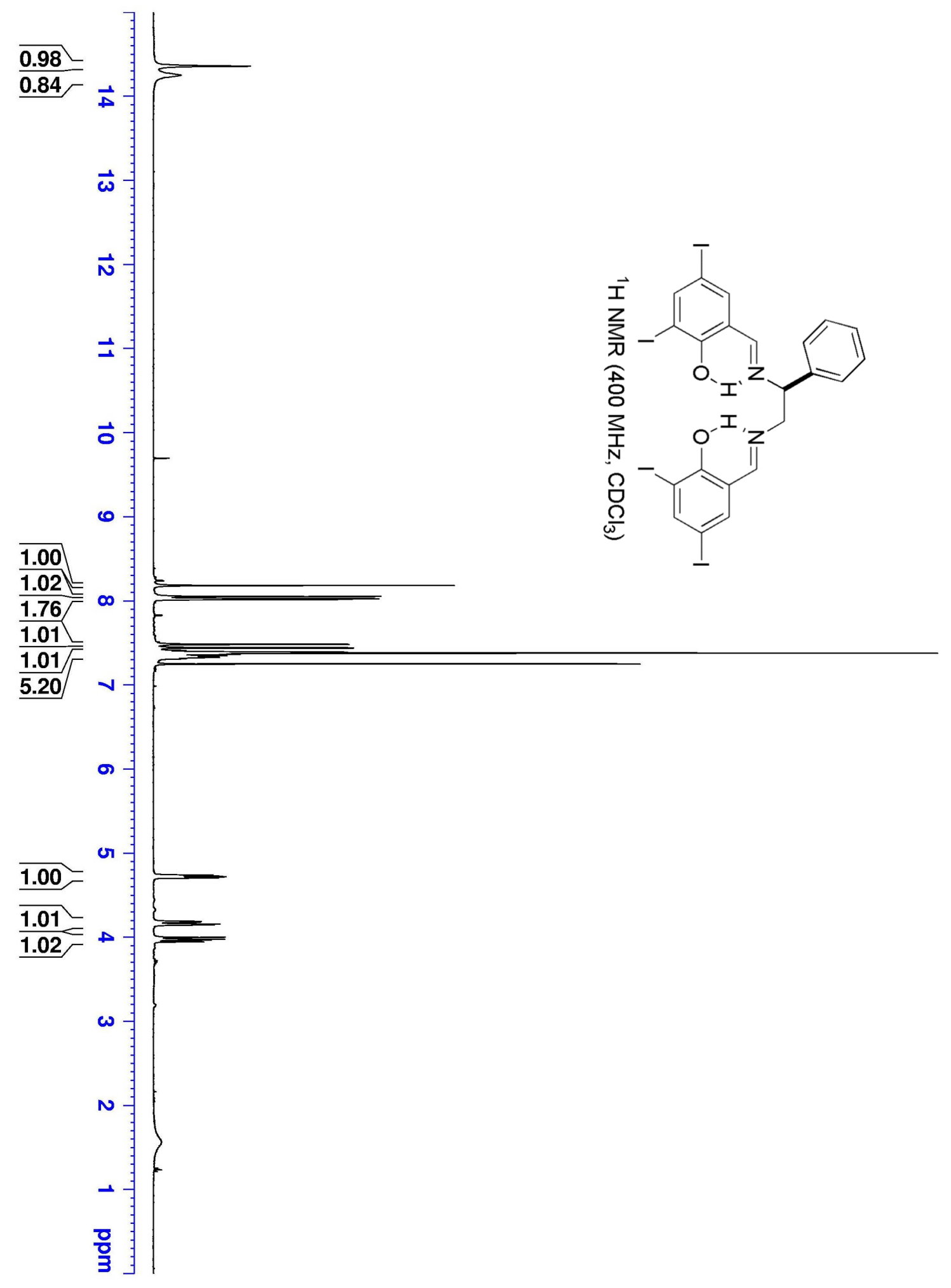




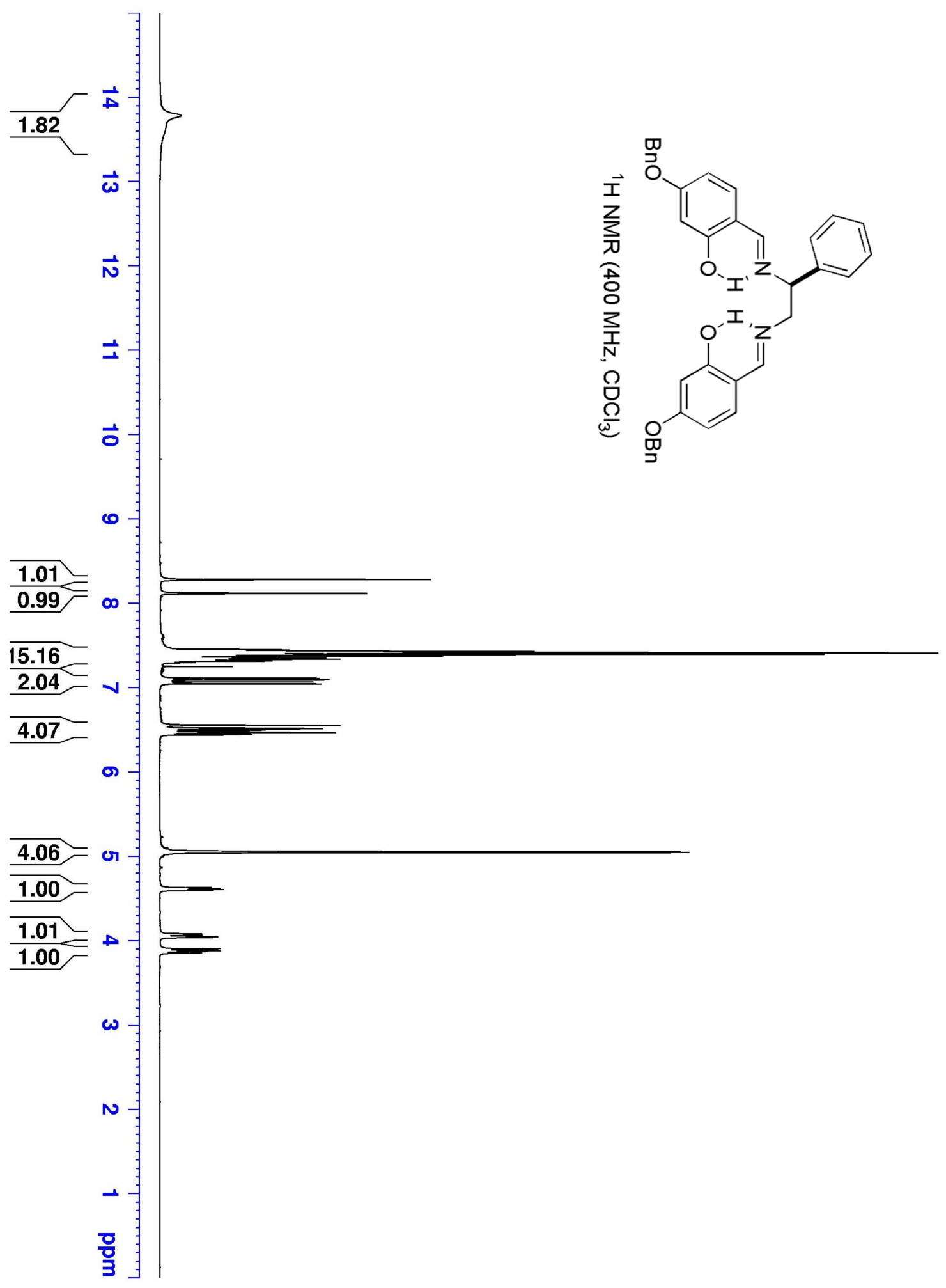




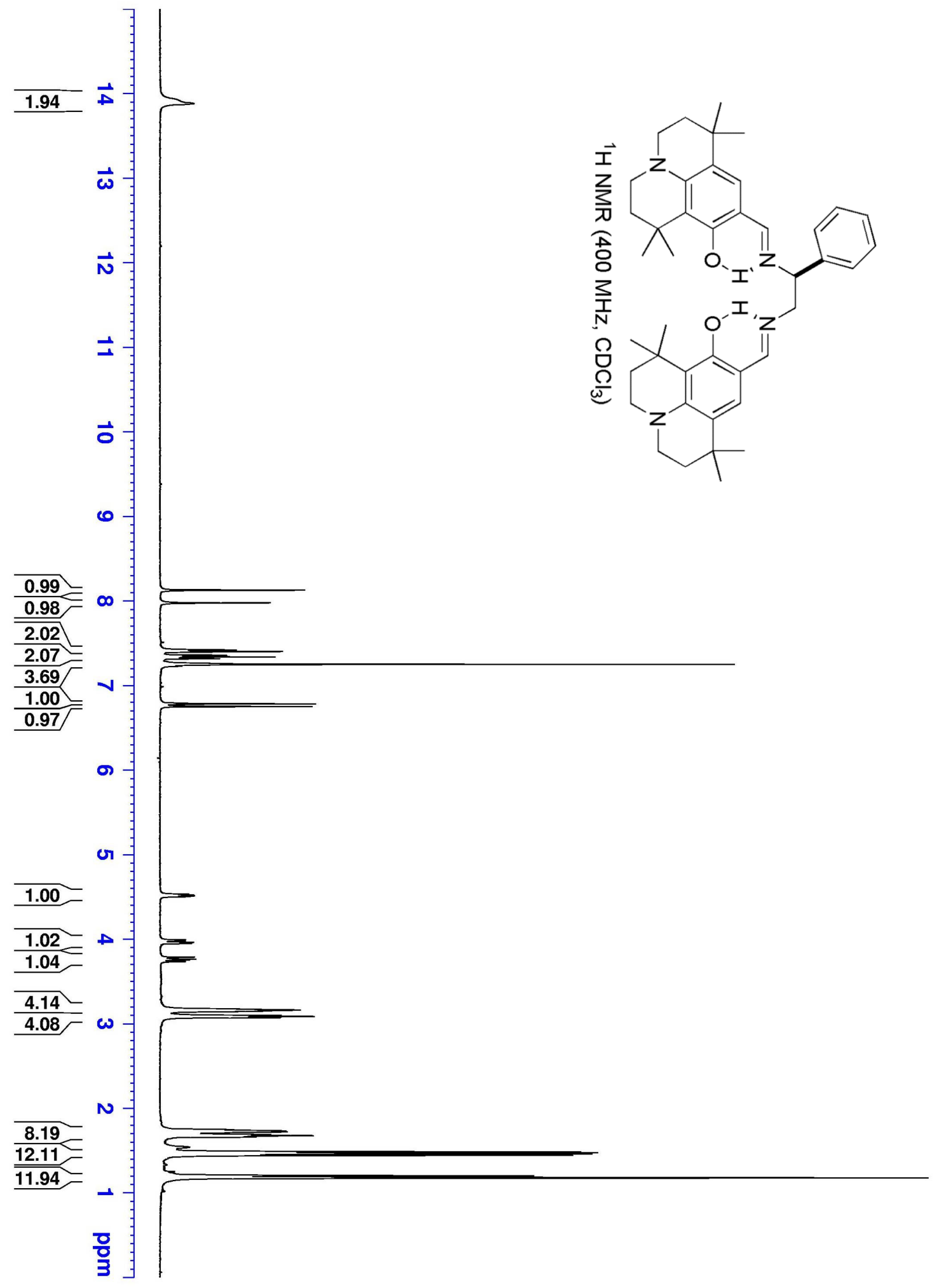




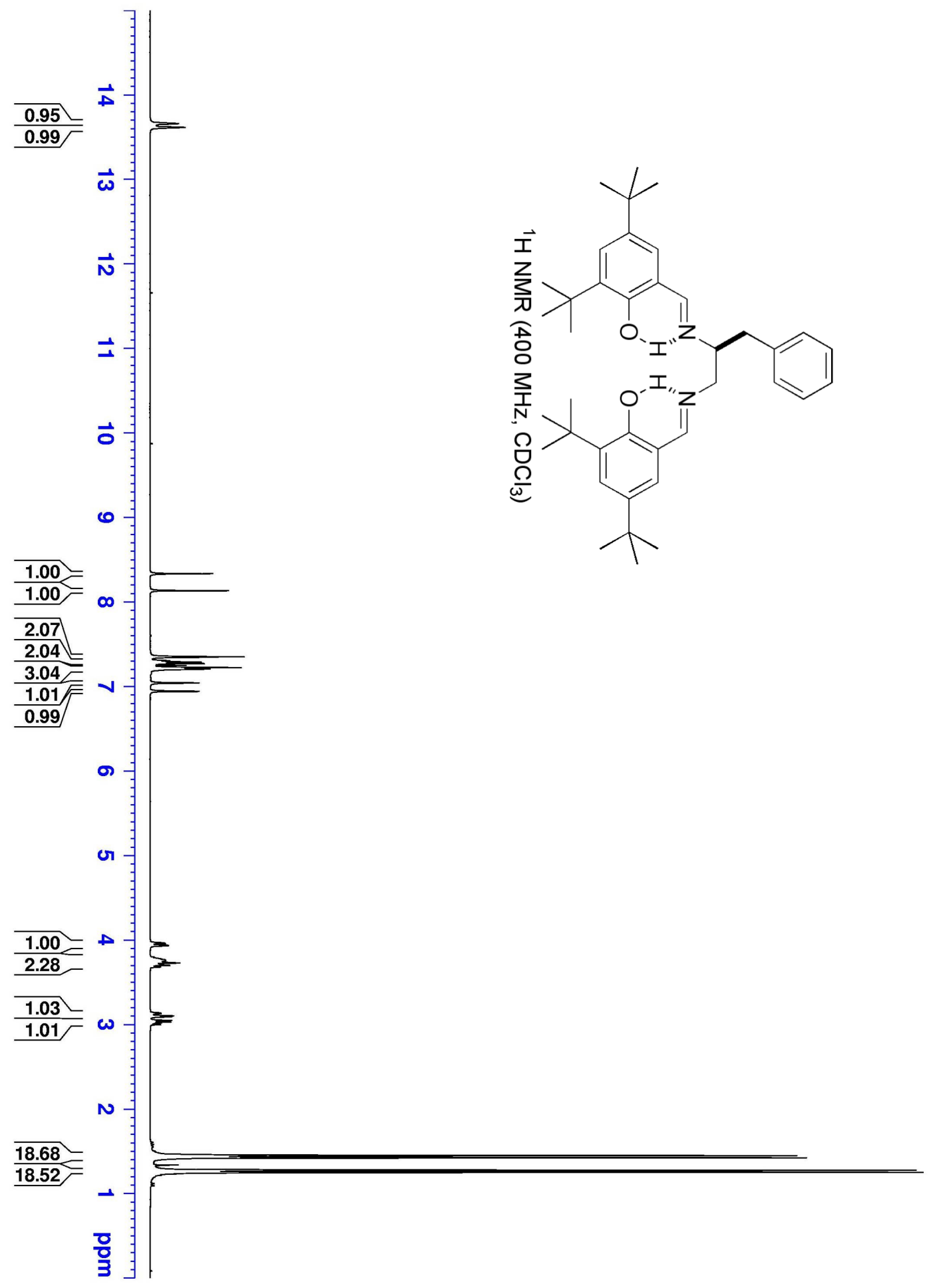




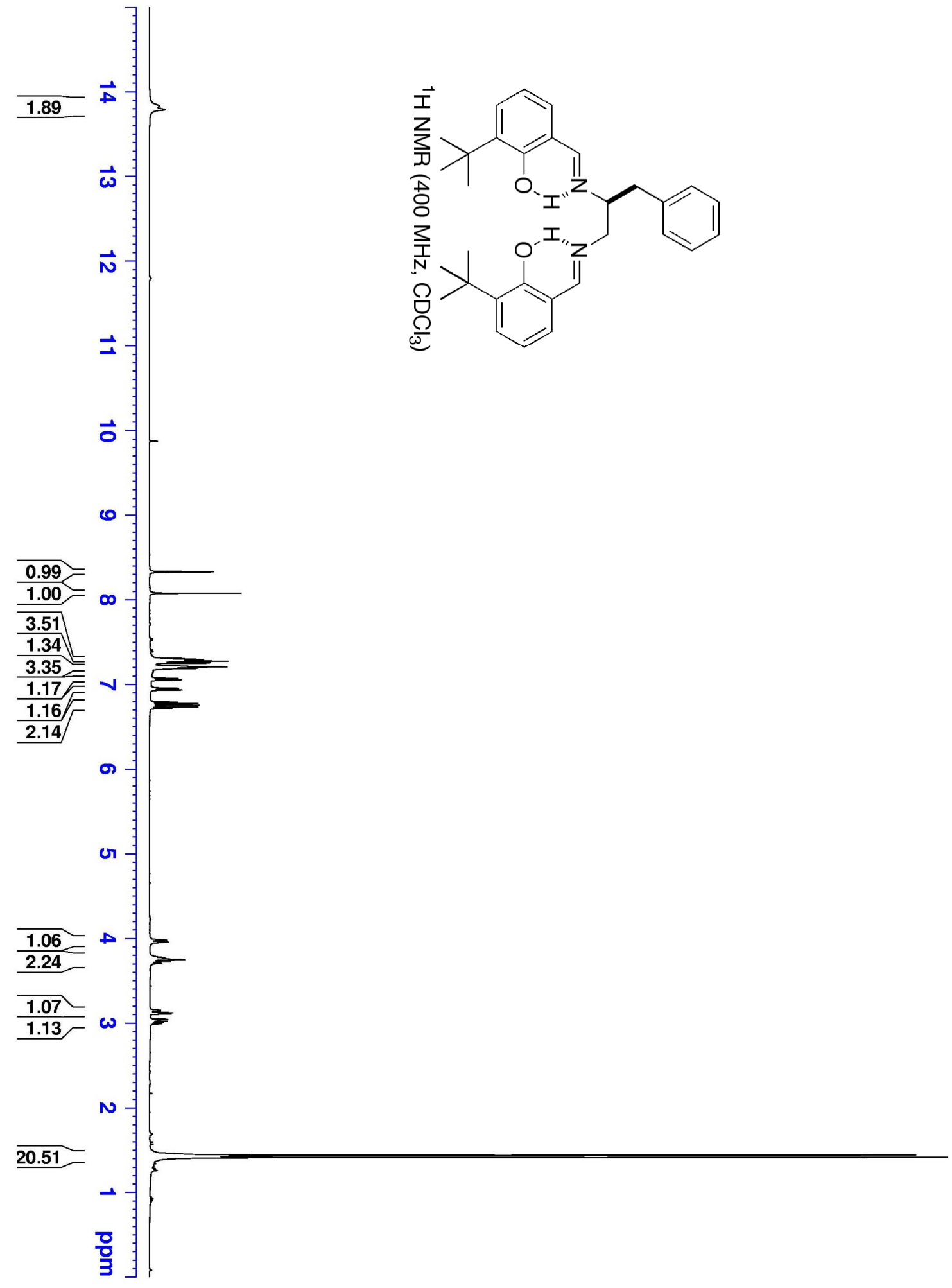




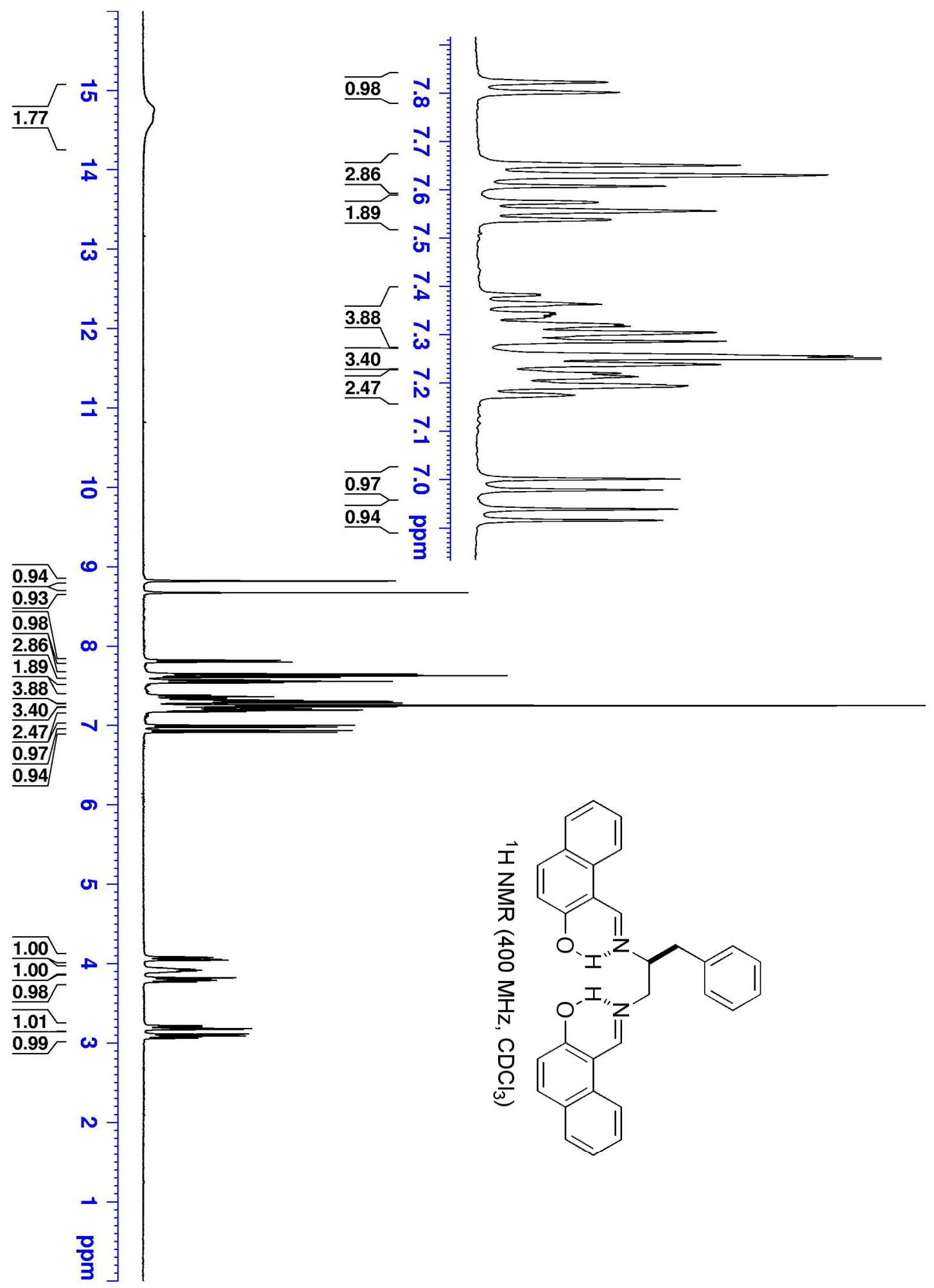




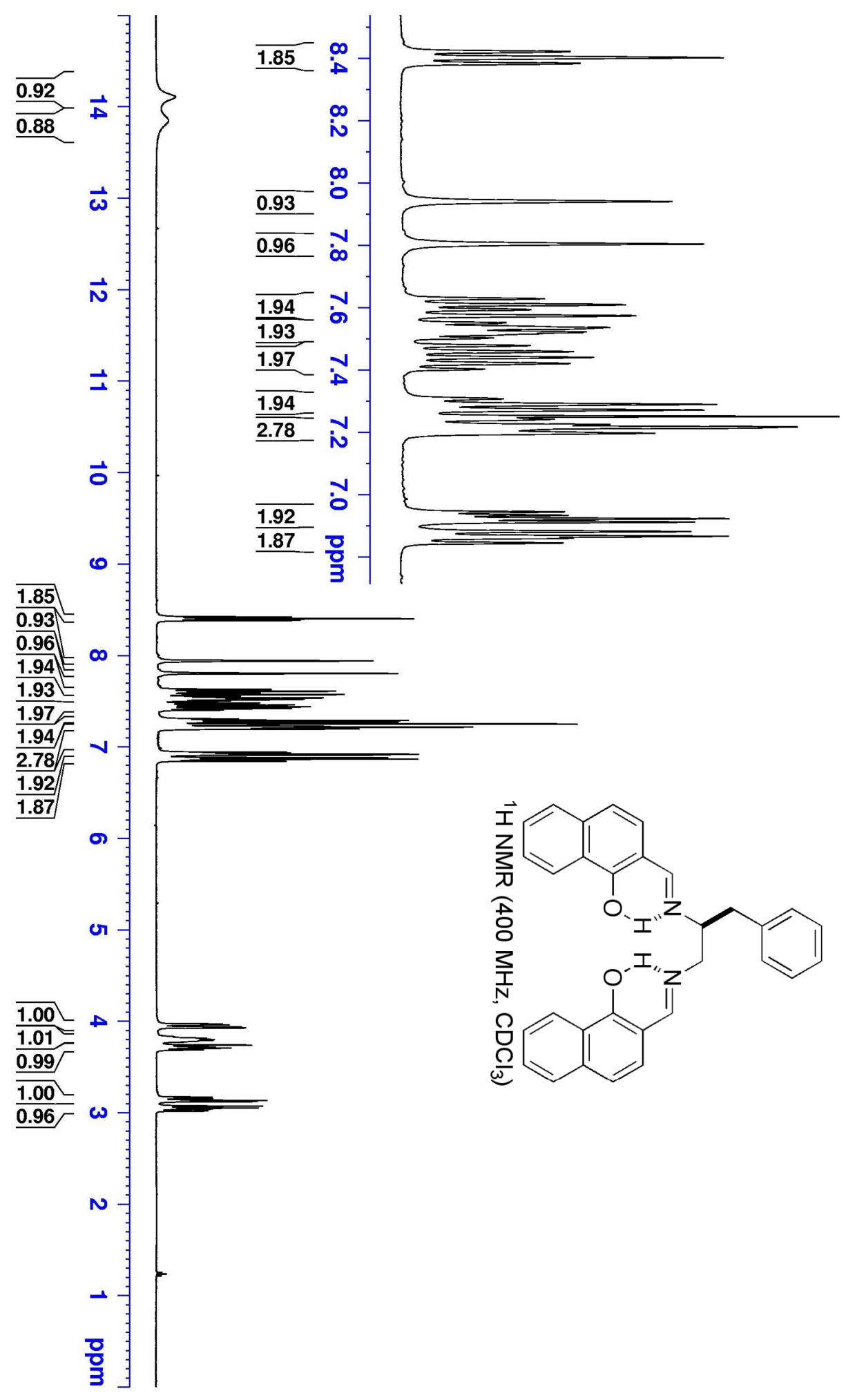




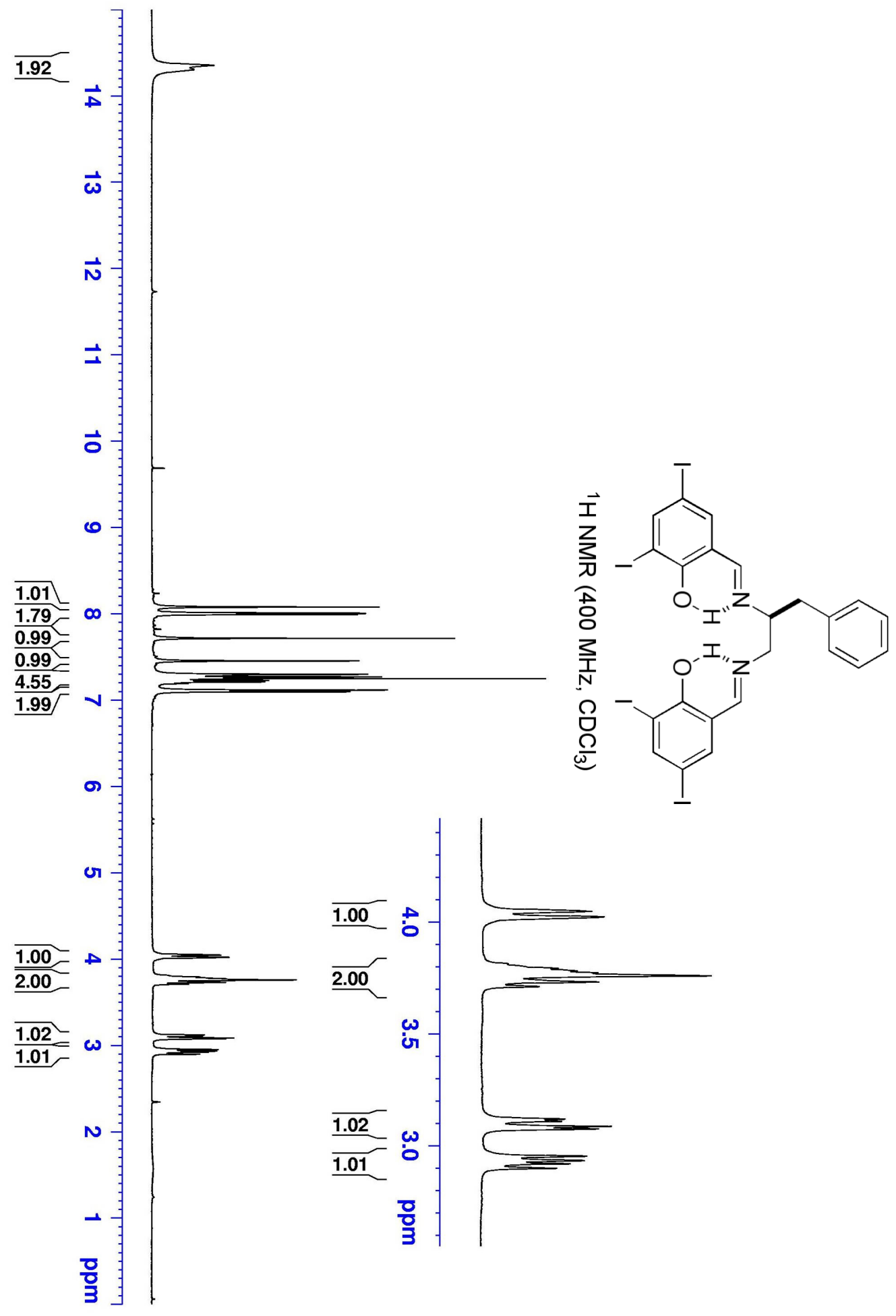




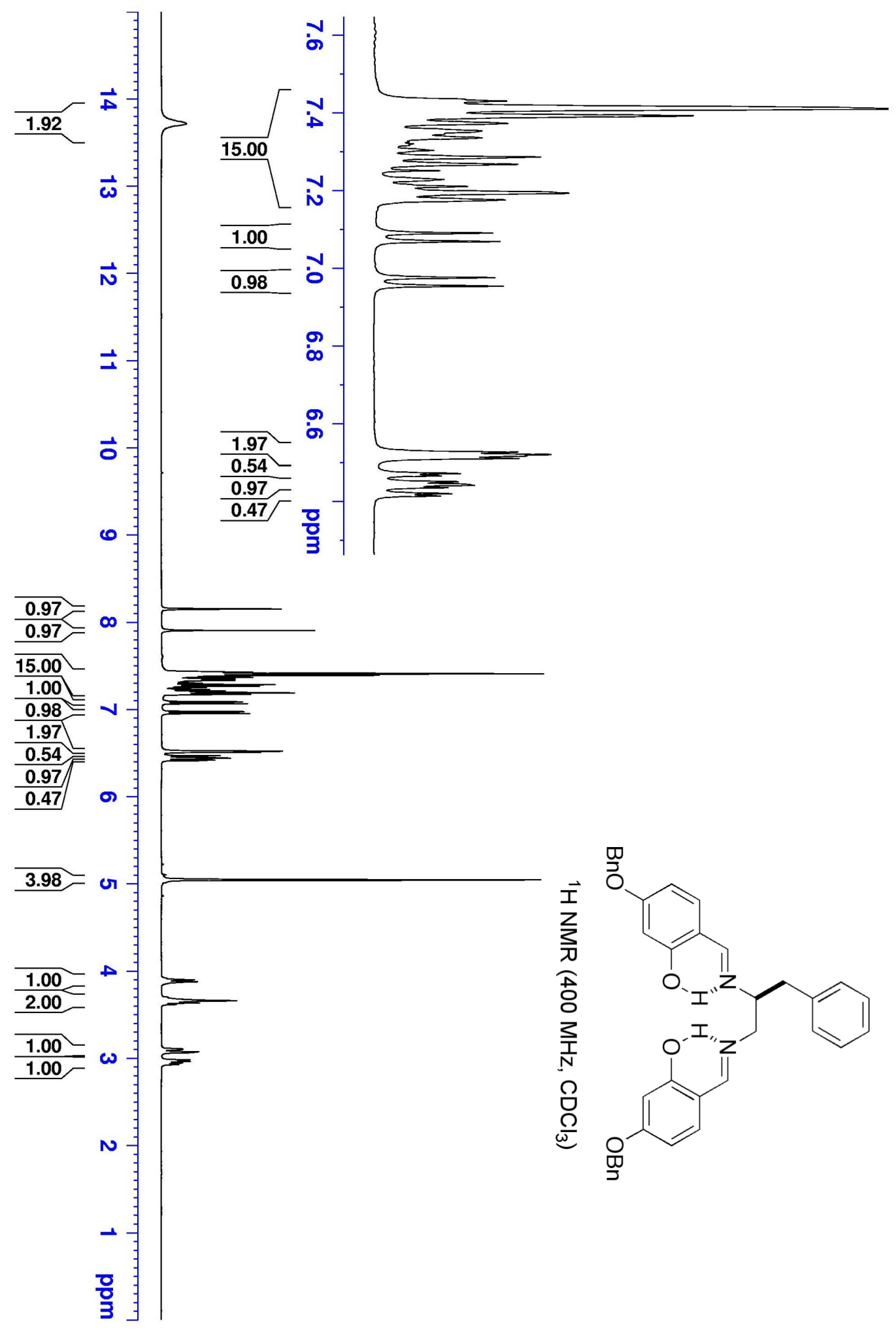




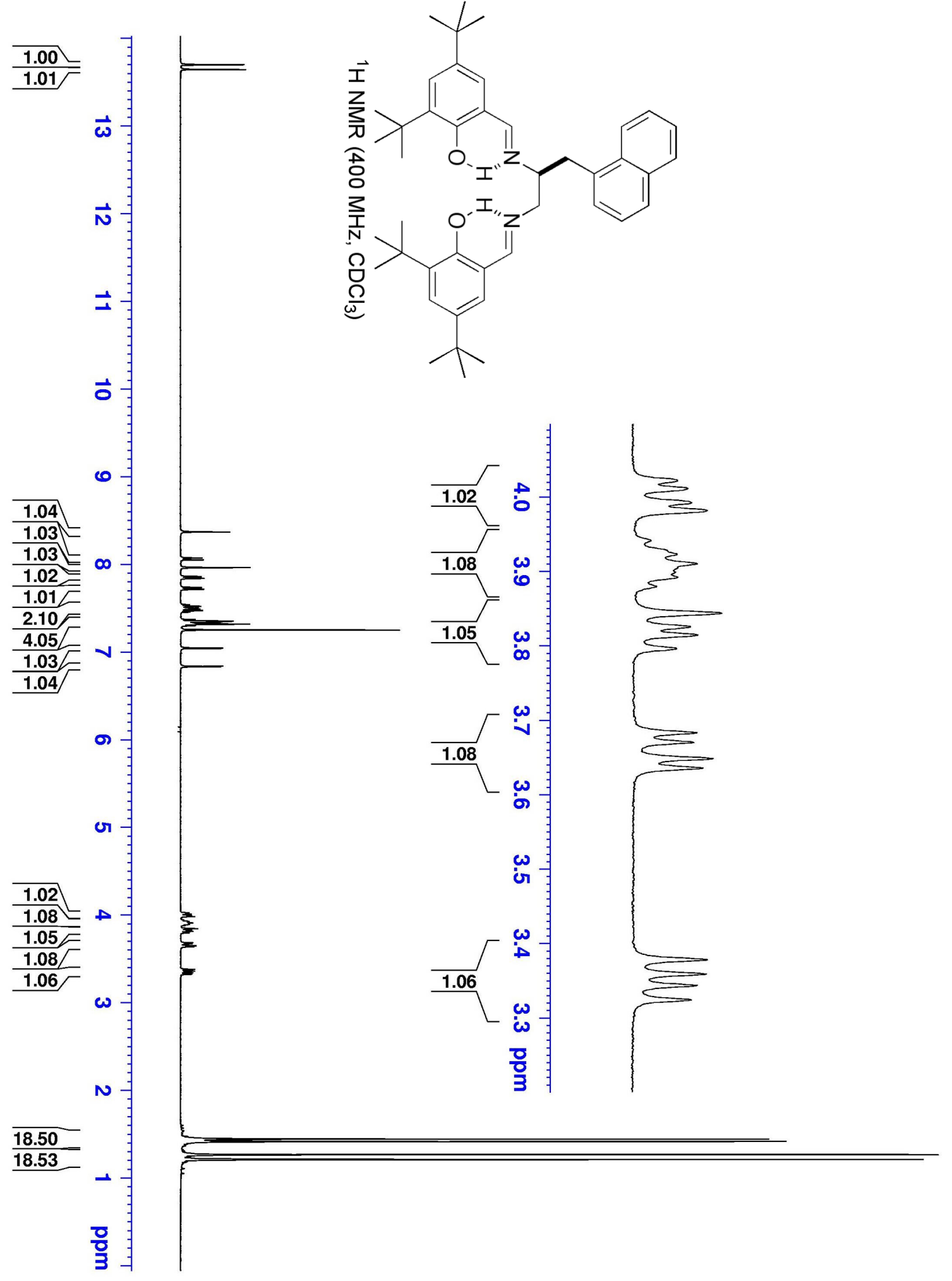




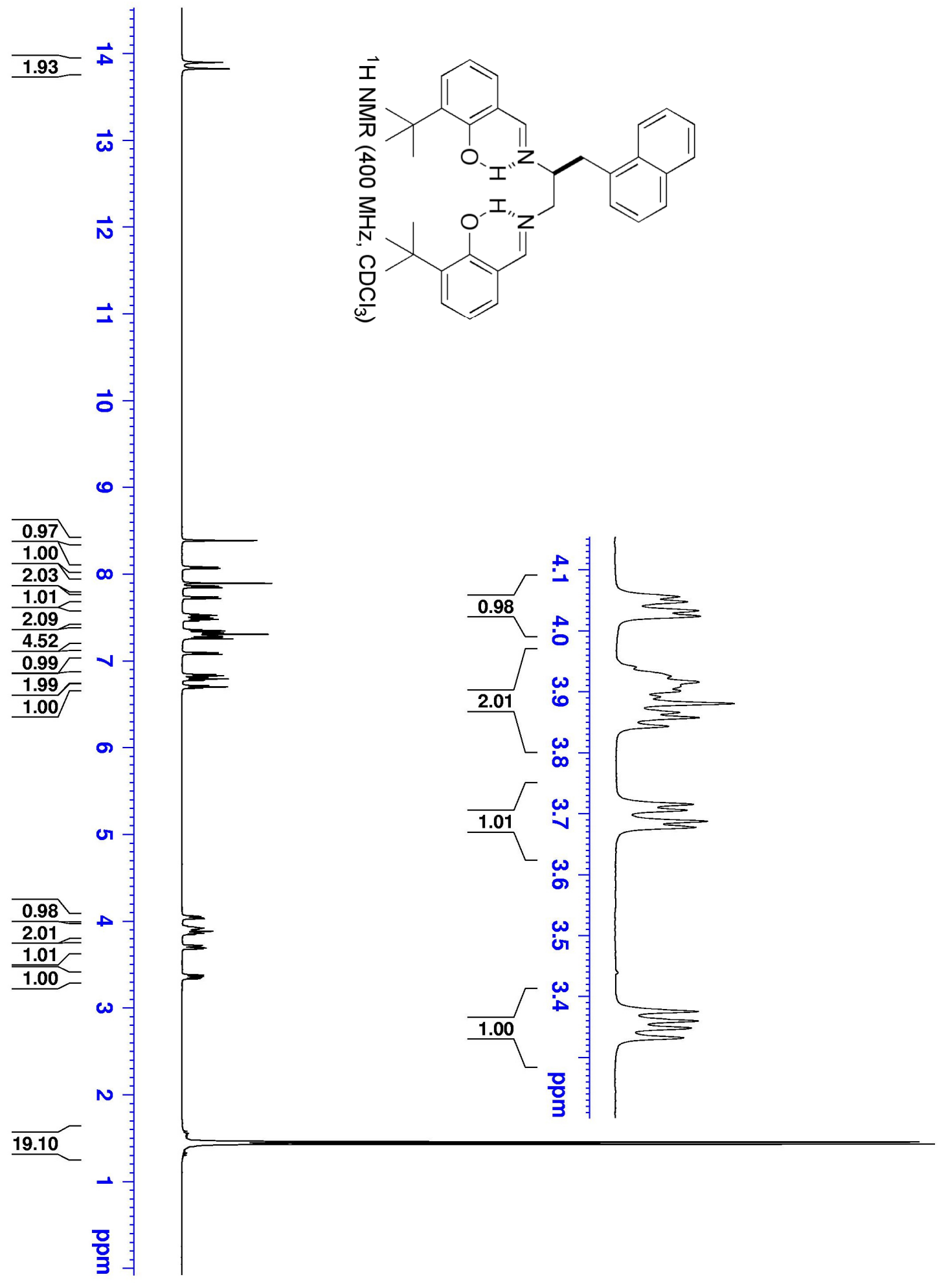




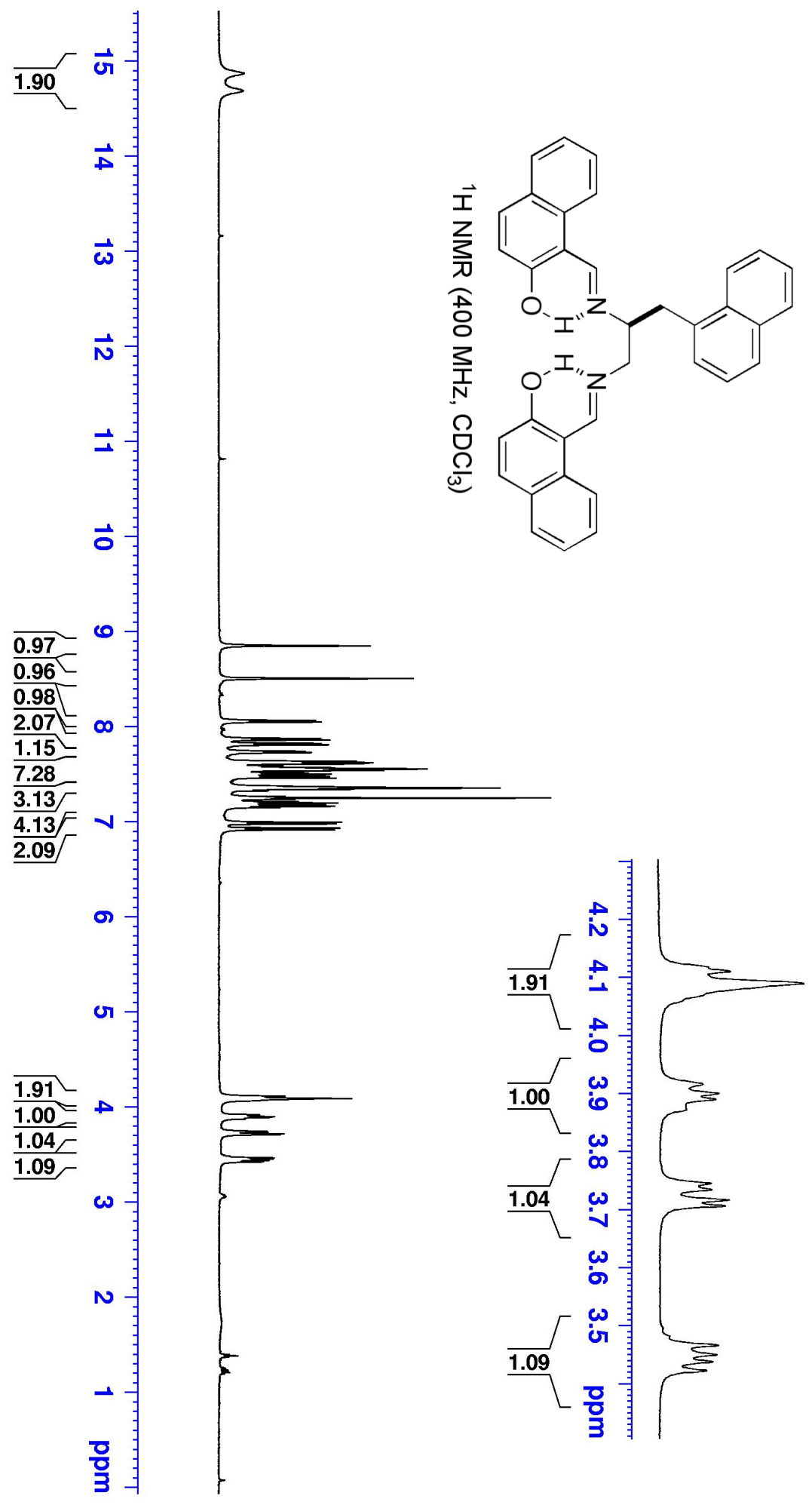




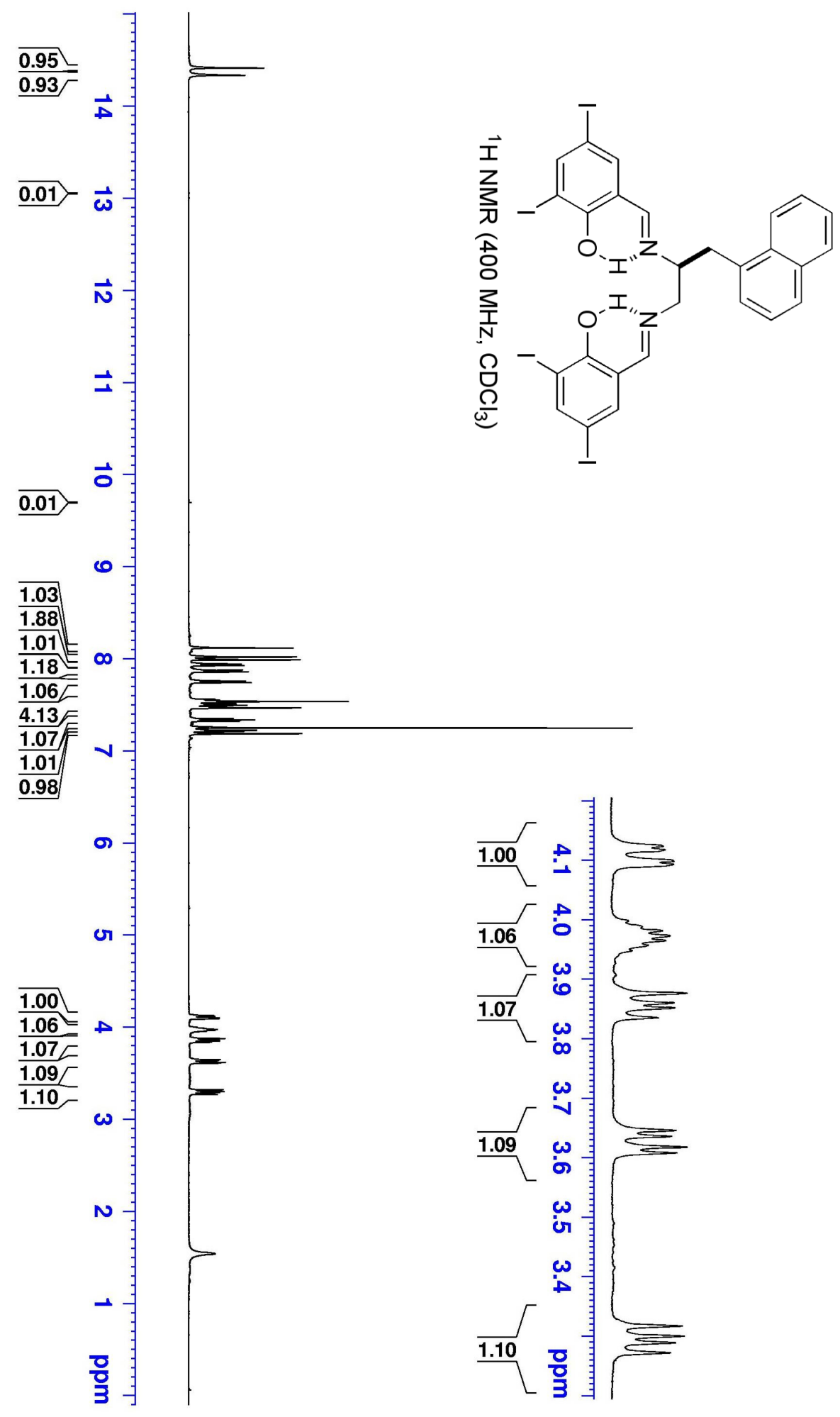




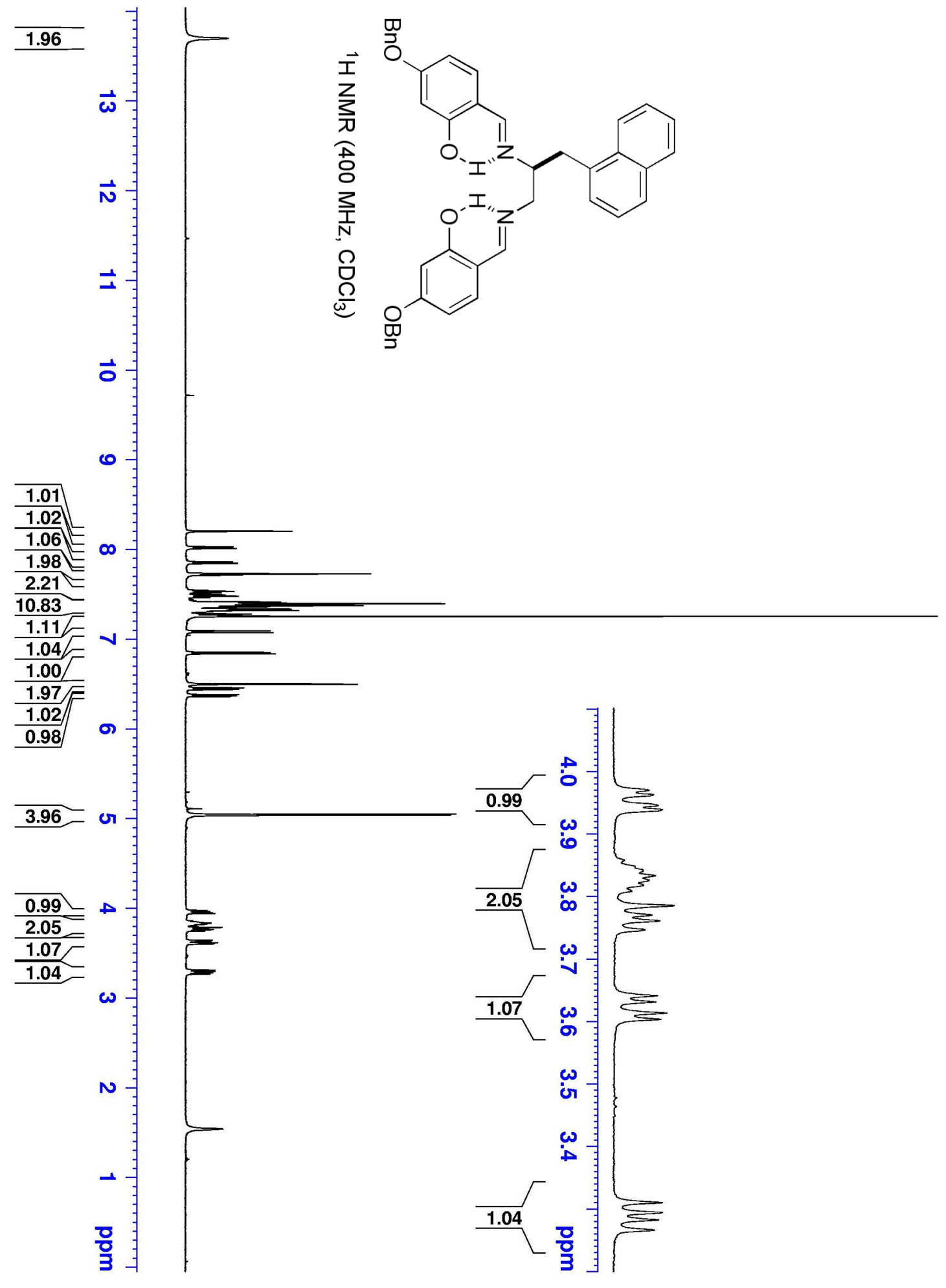




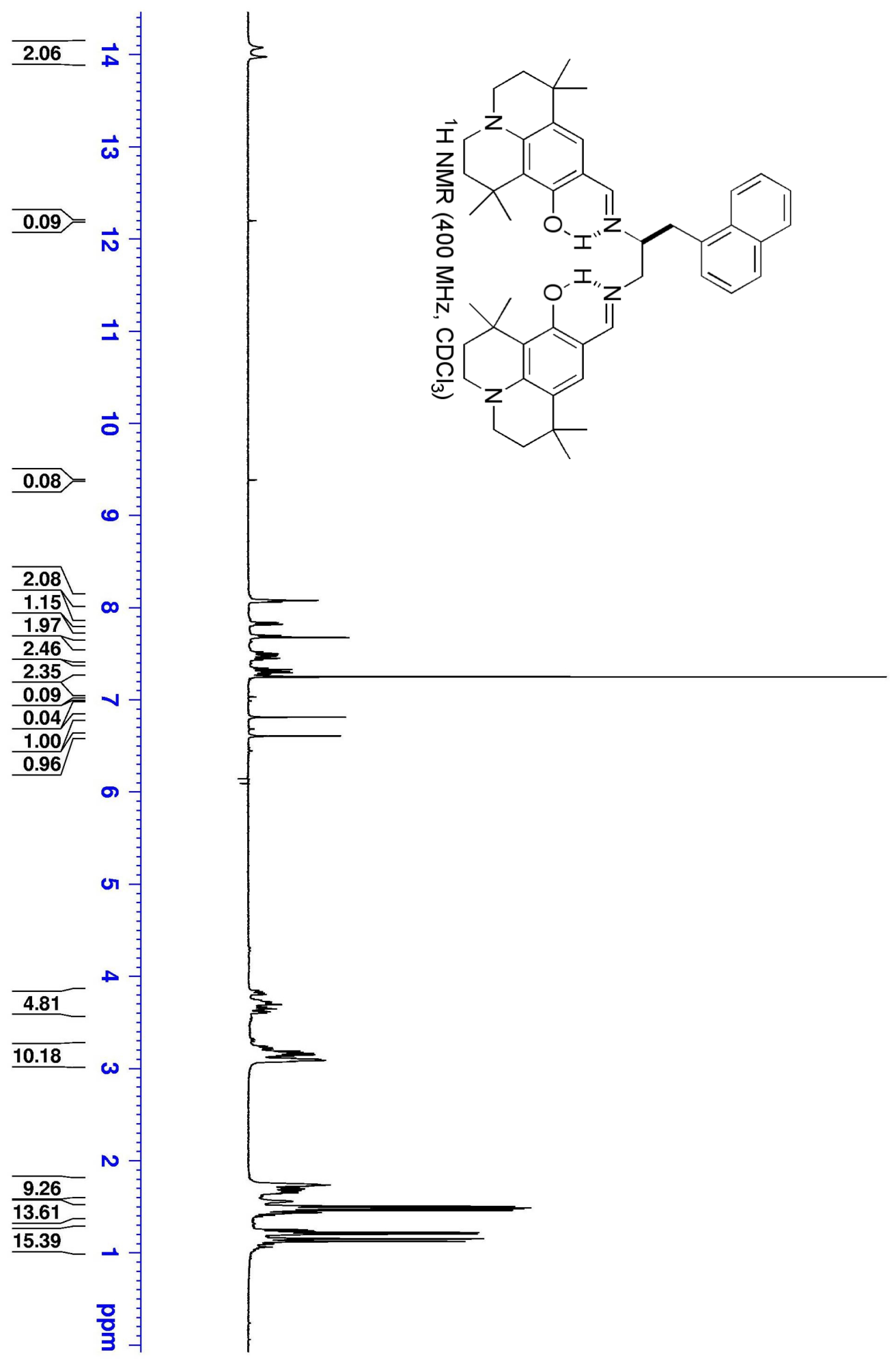




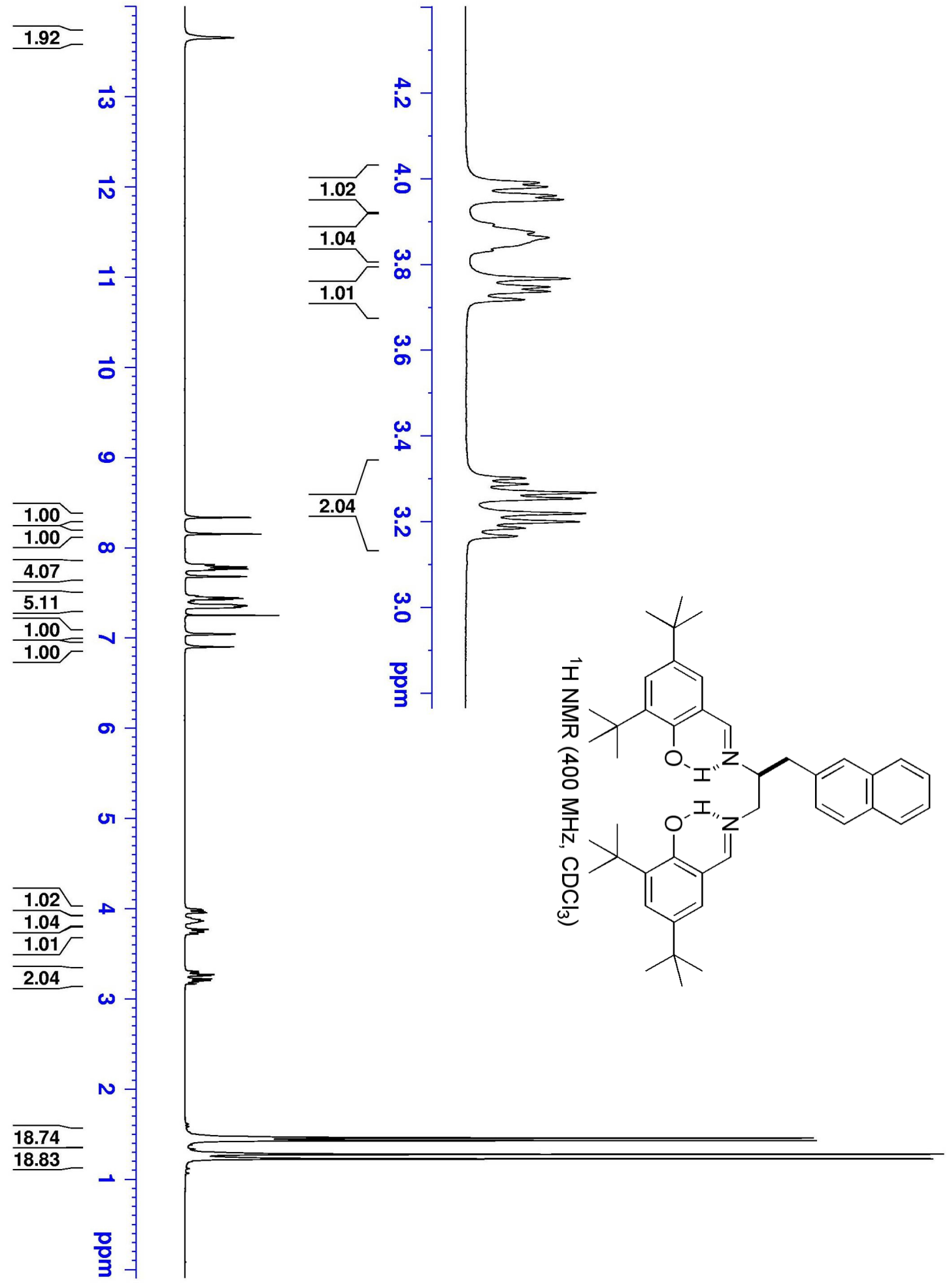




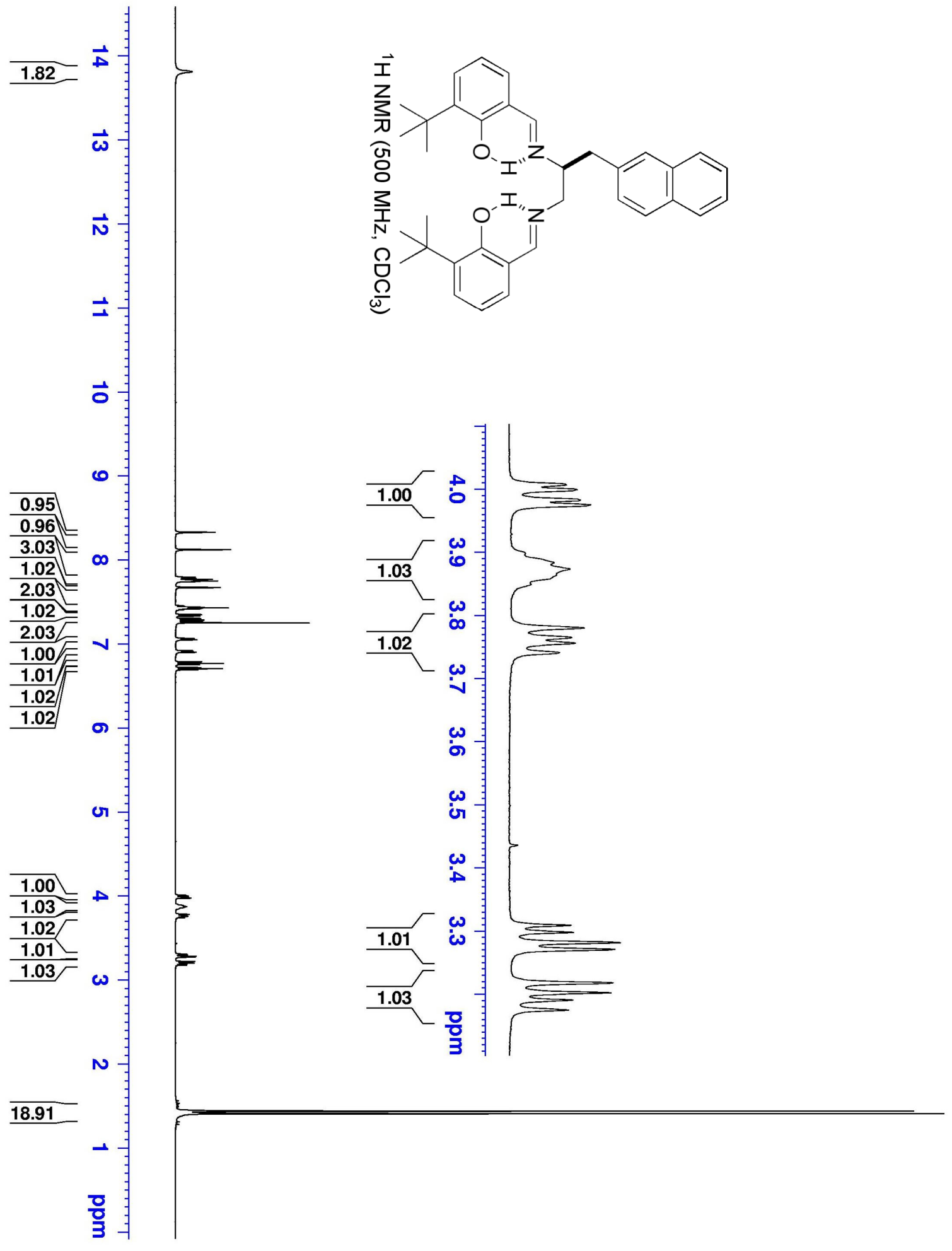




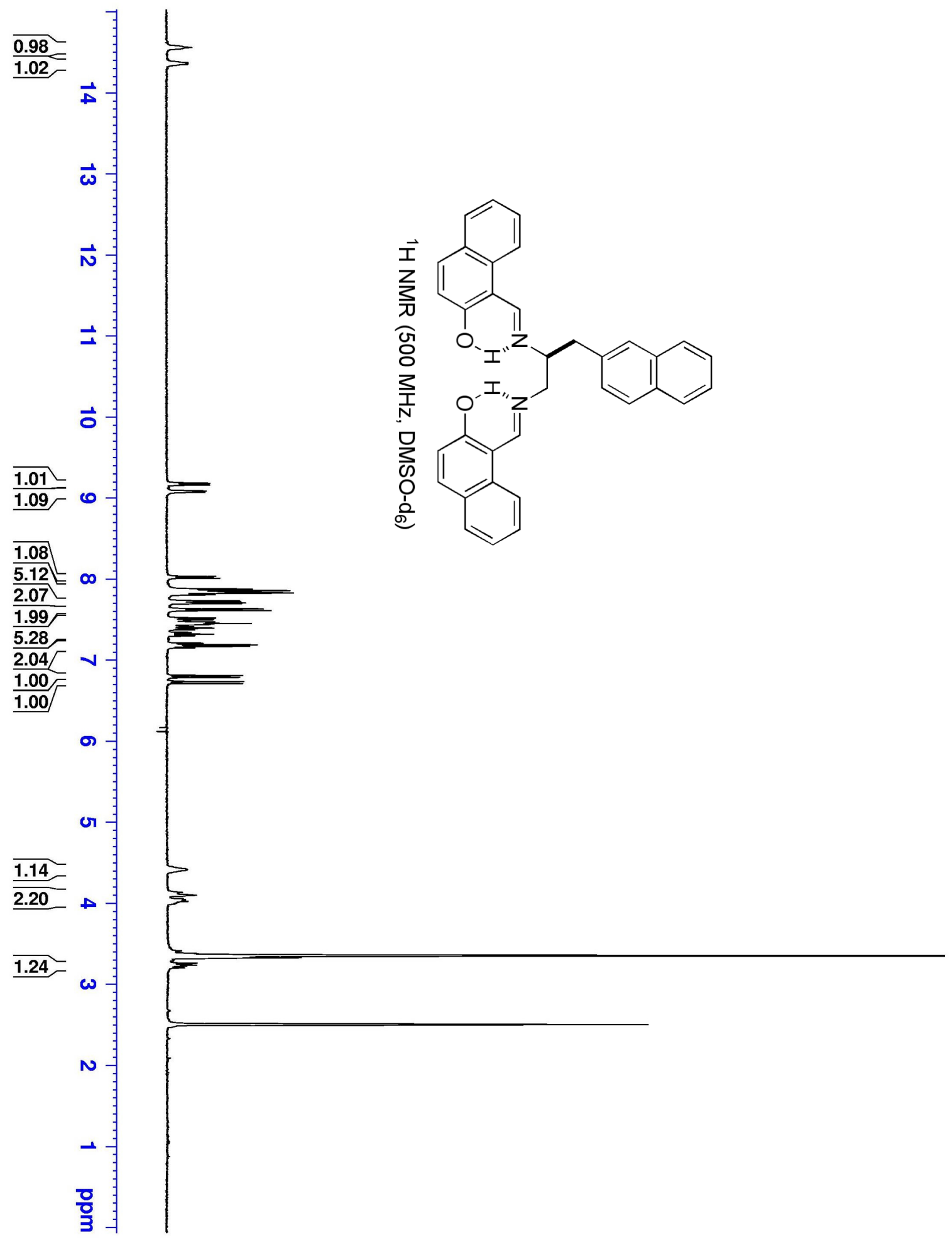

S61 


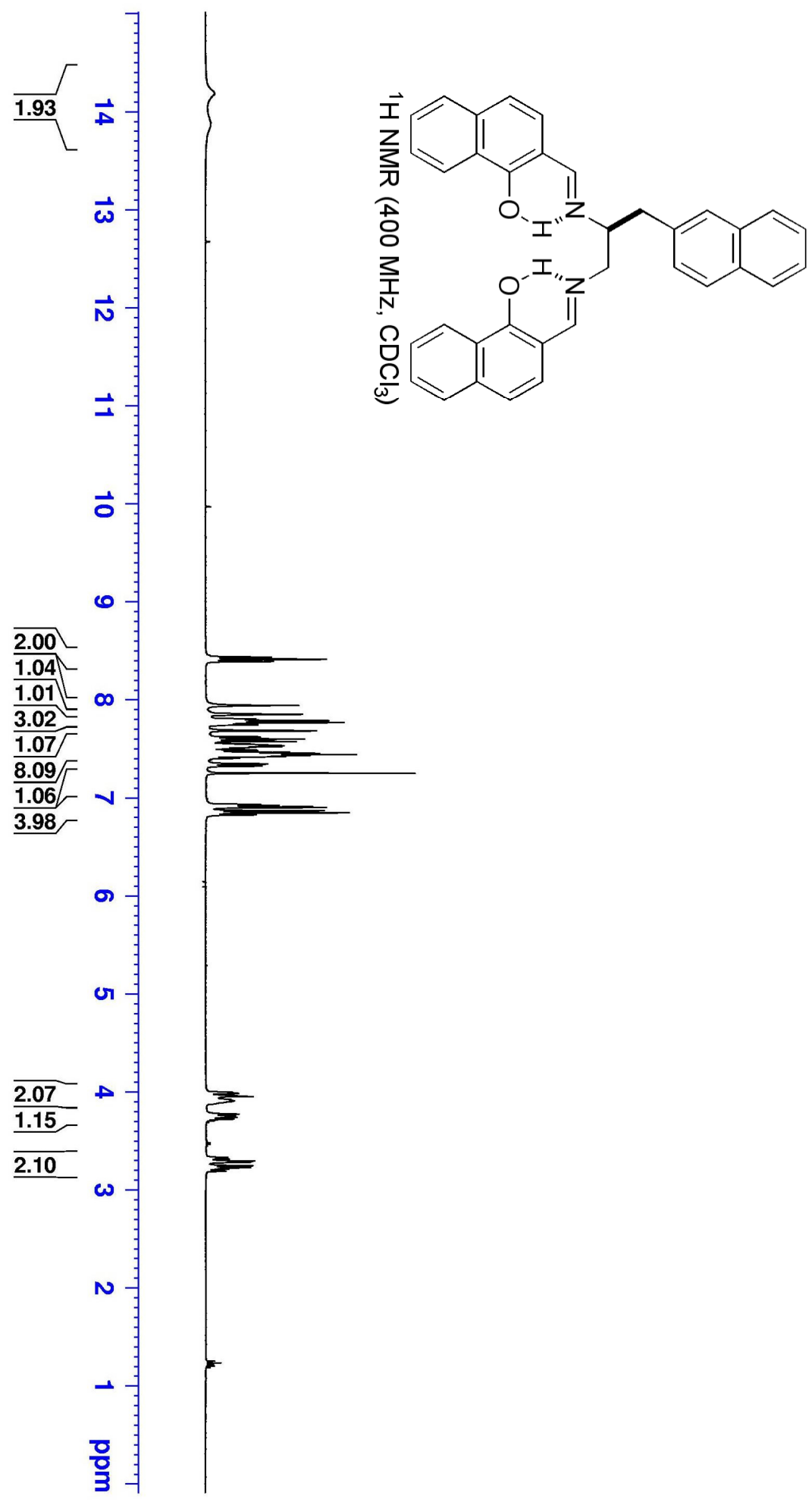




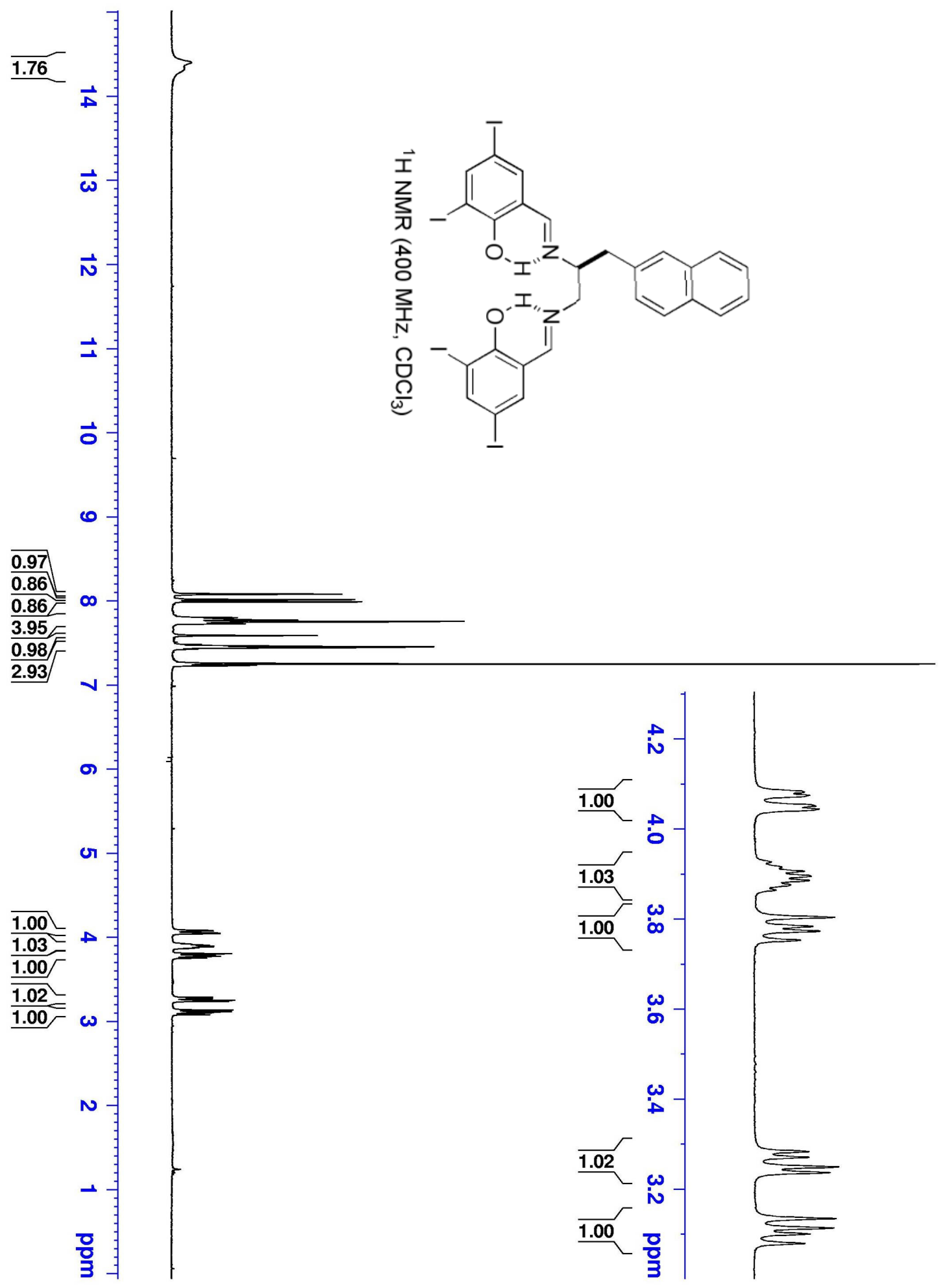




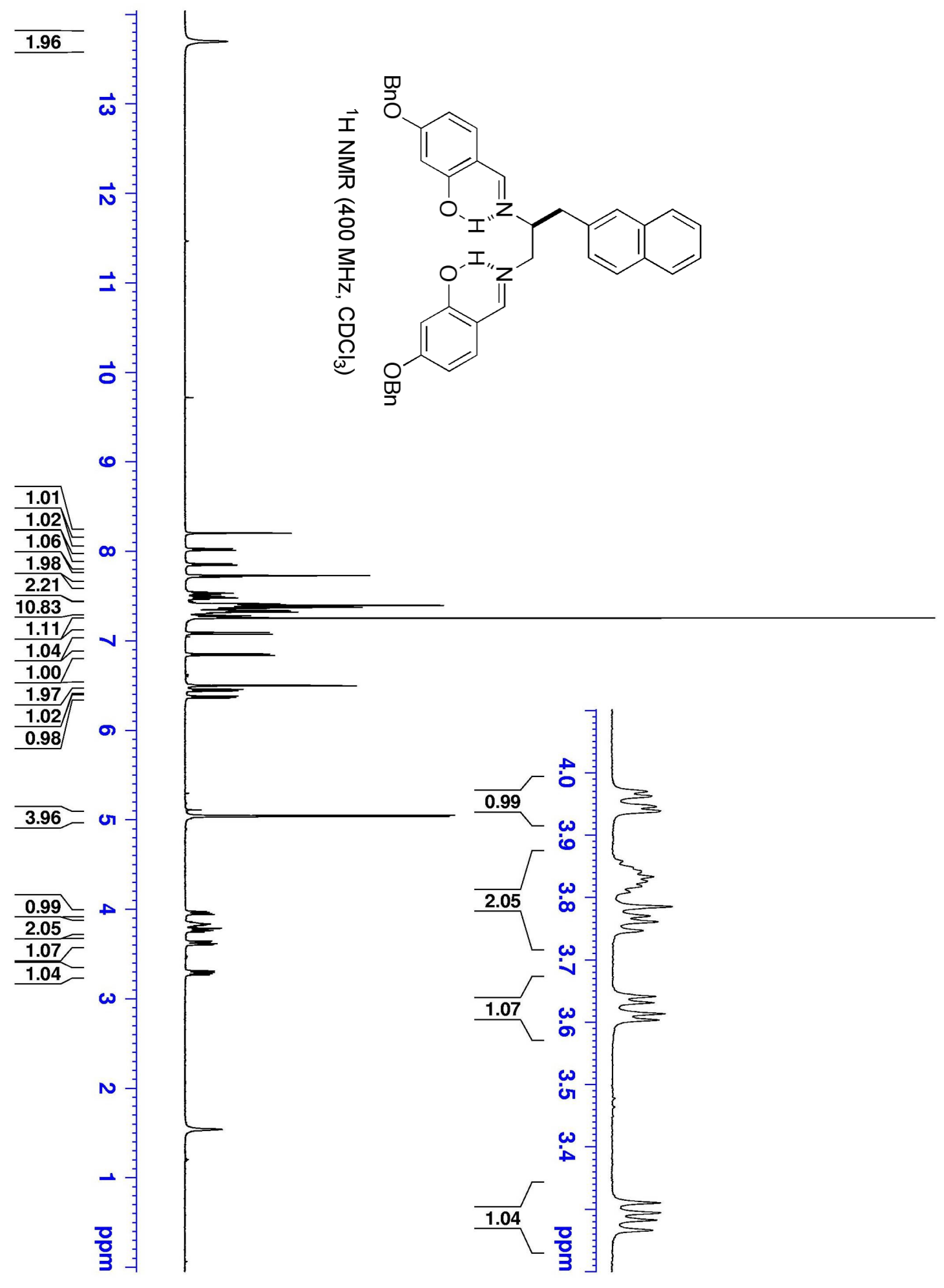




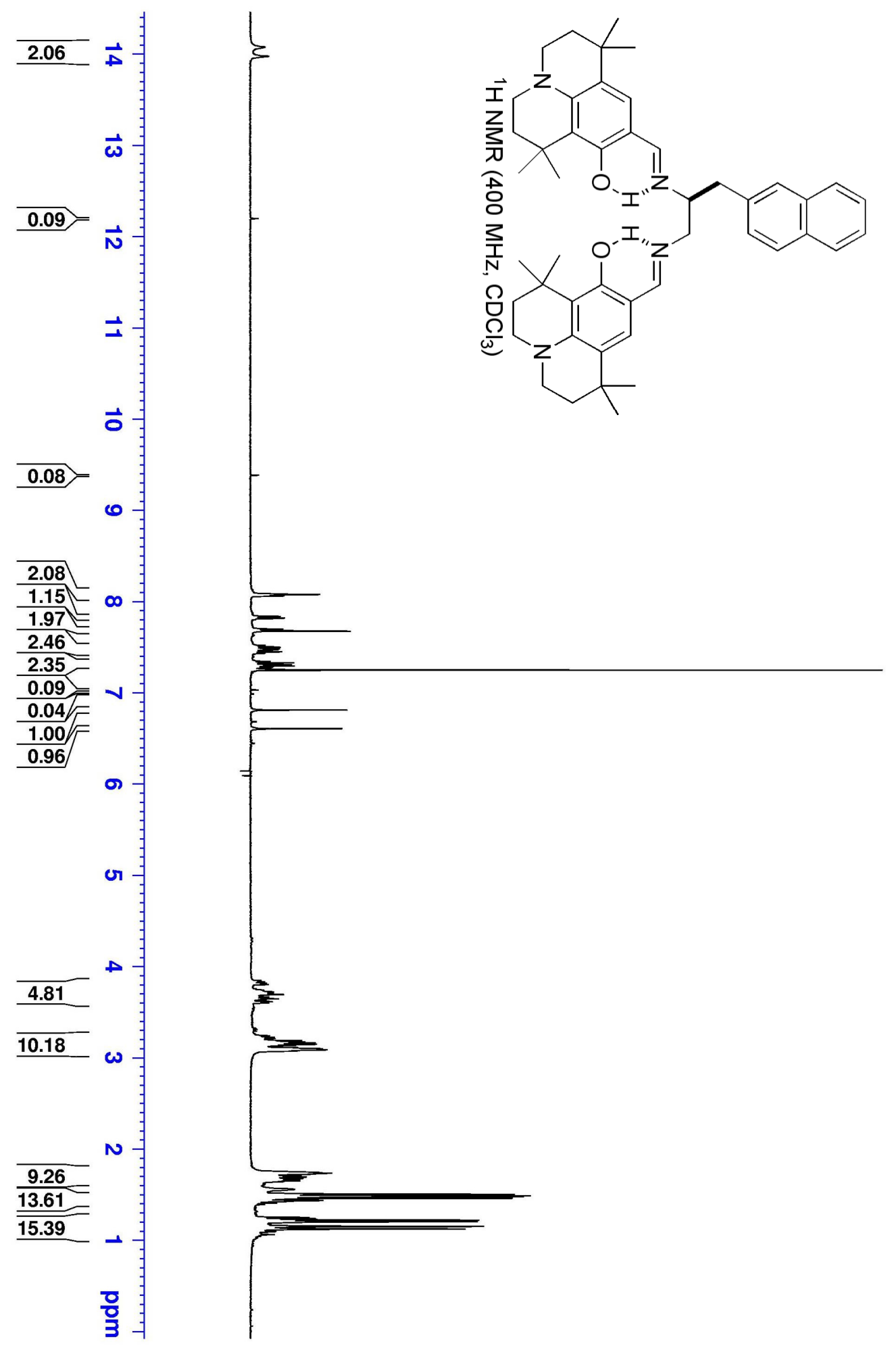




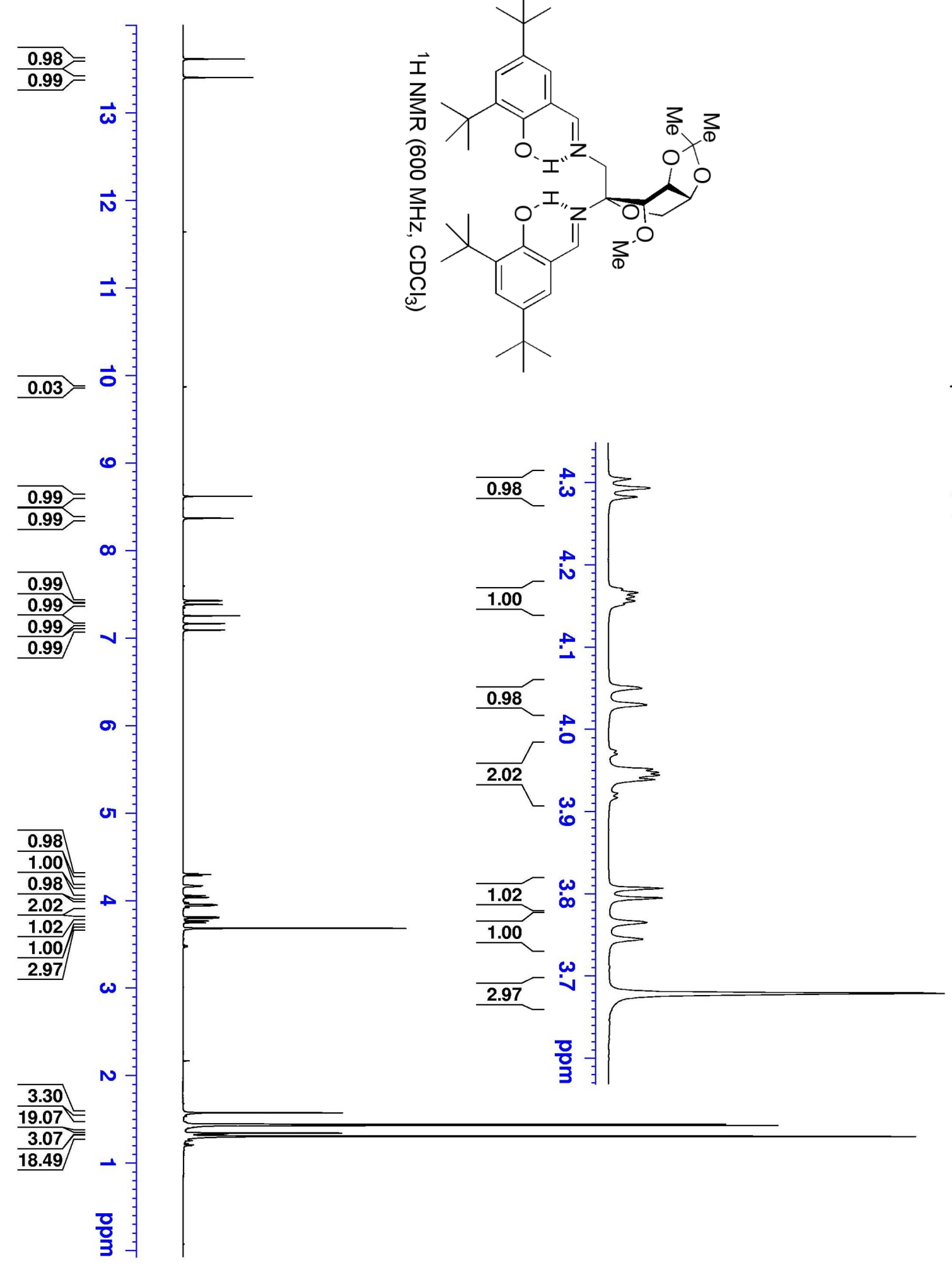




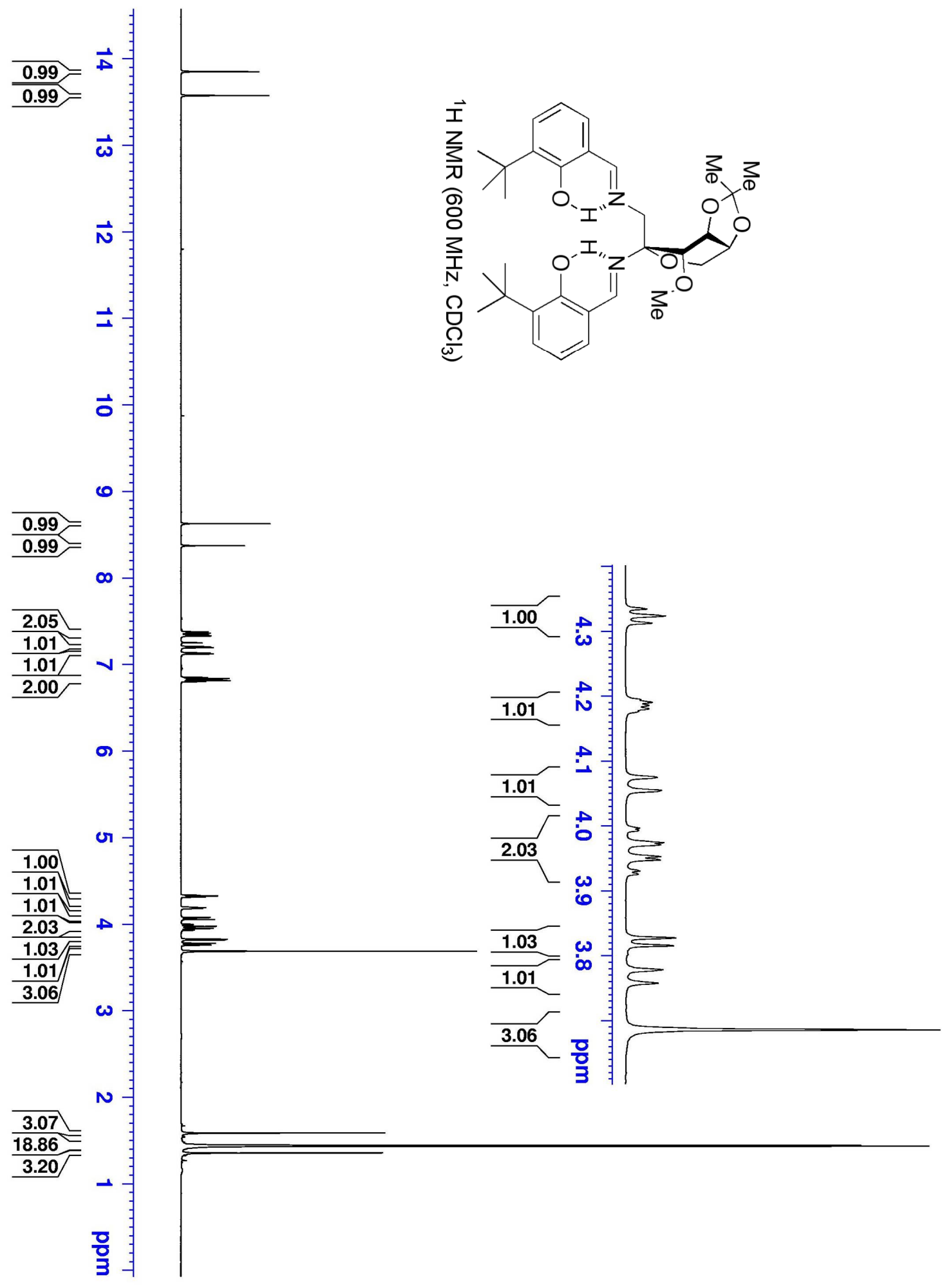




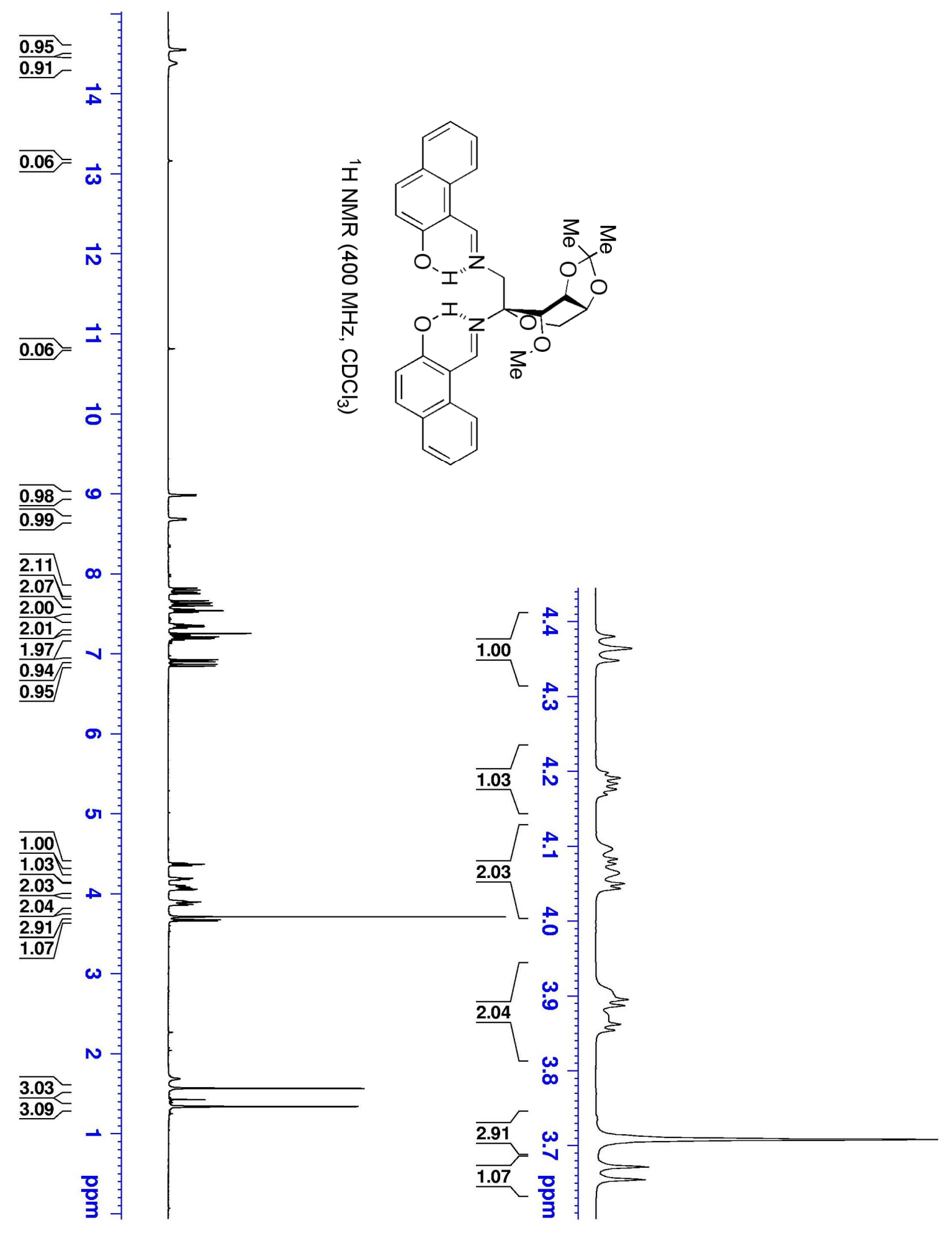




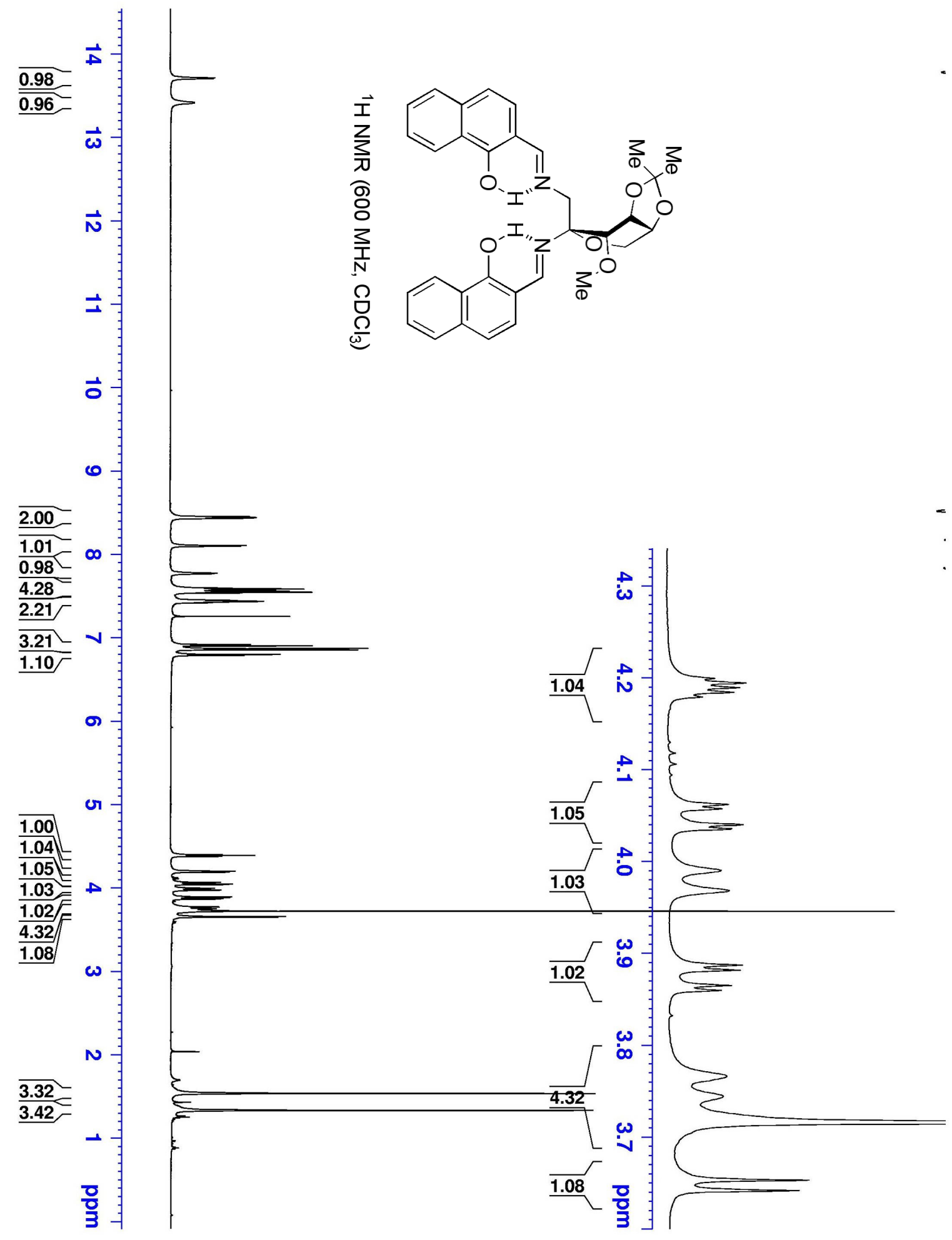




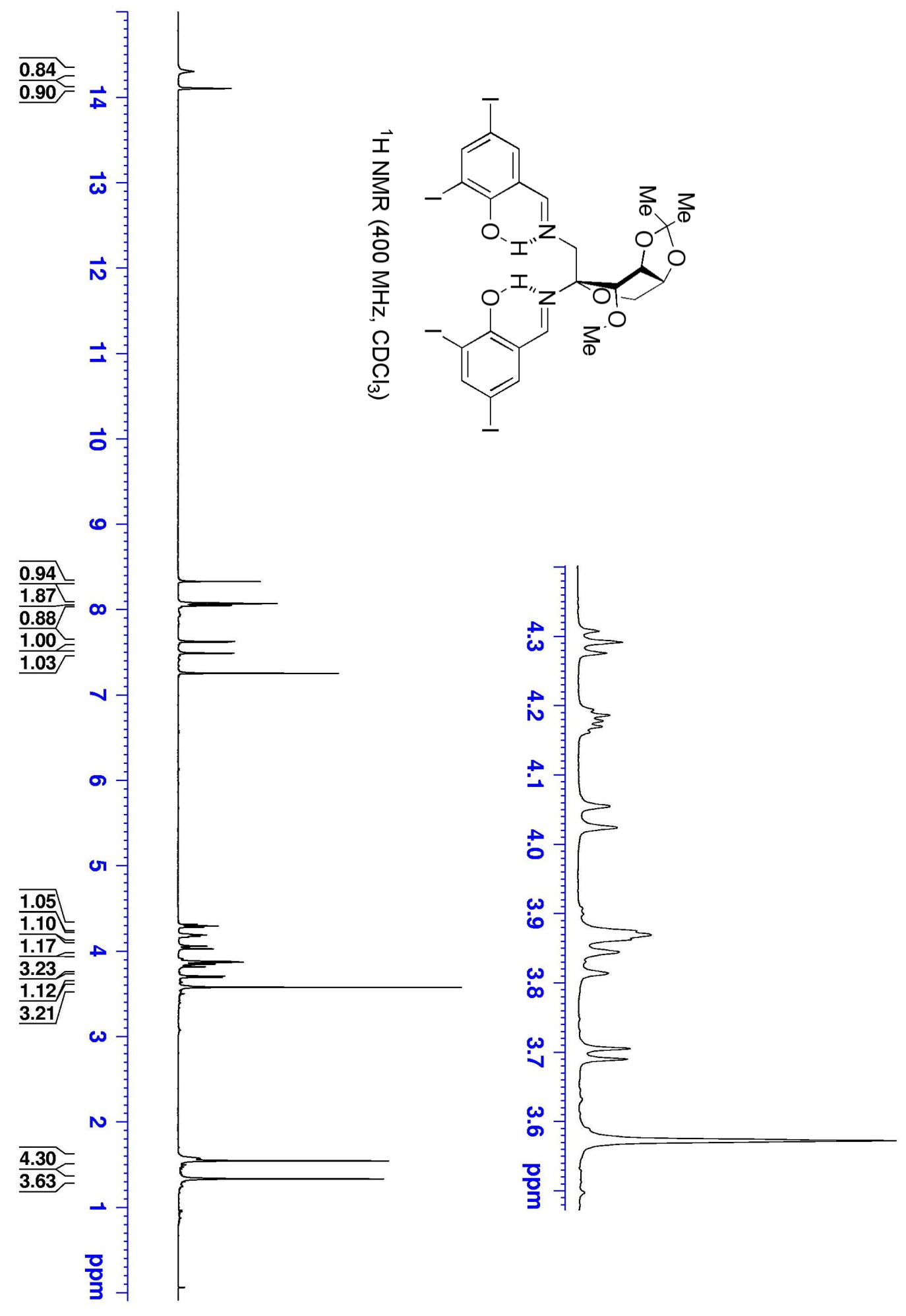




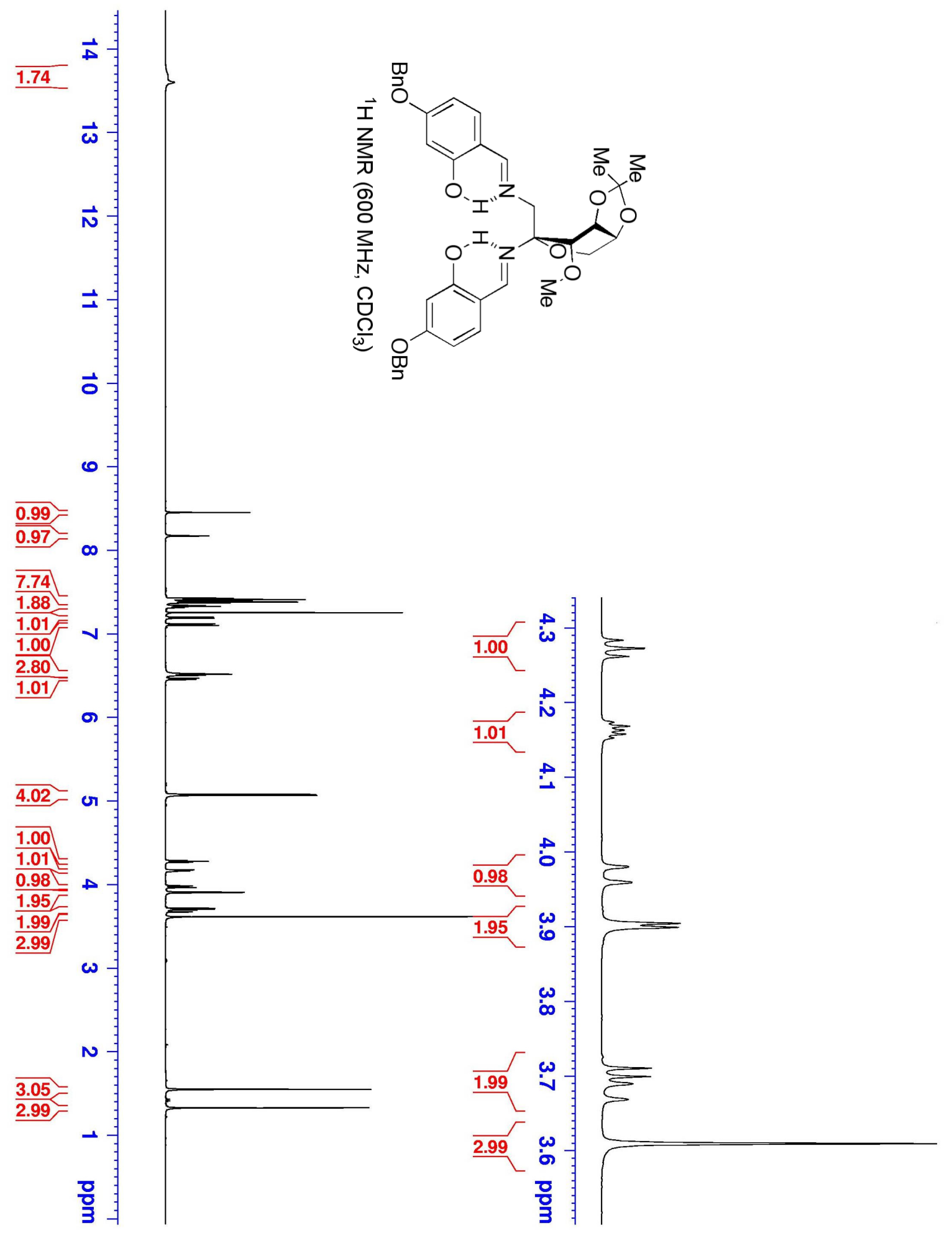




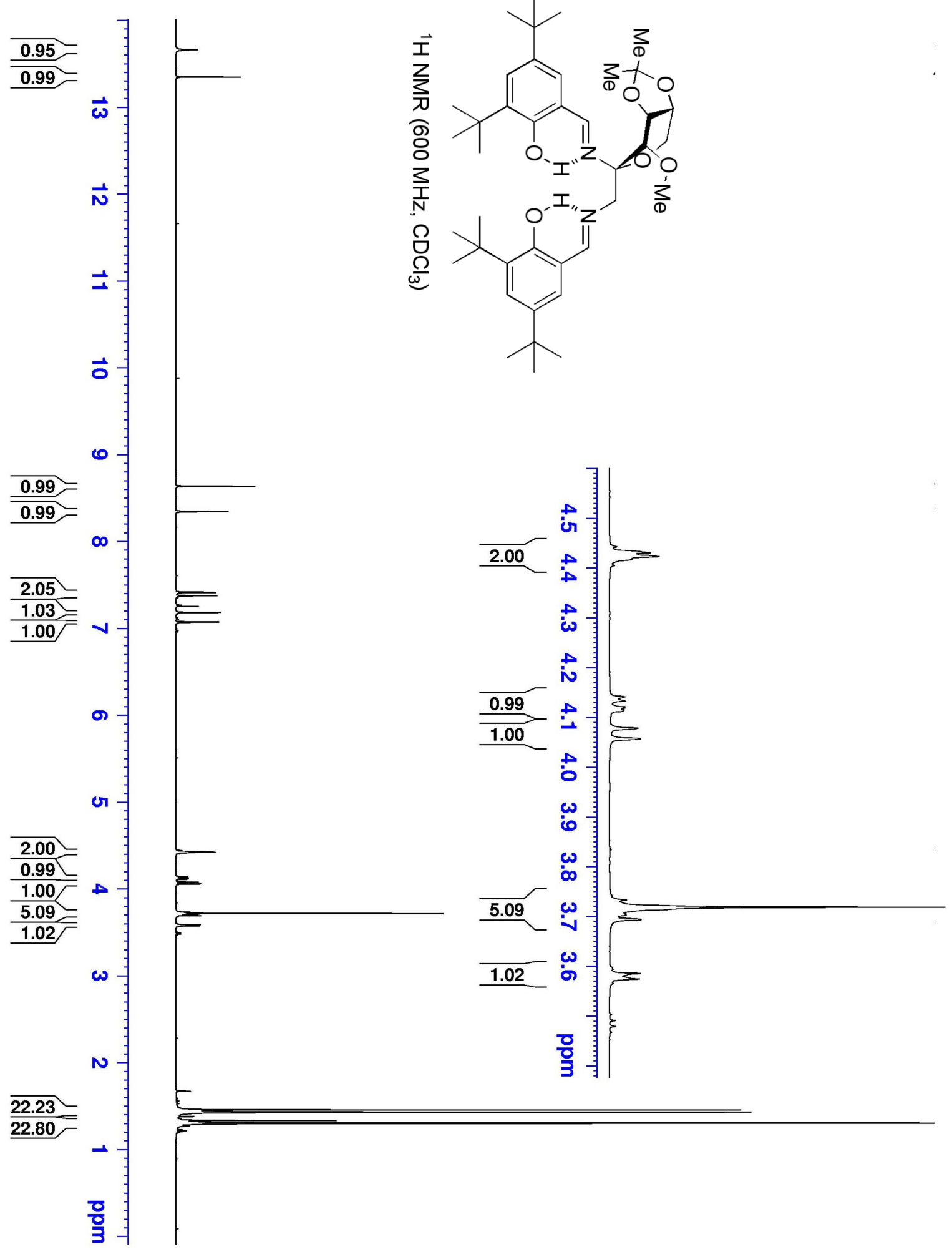




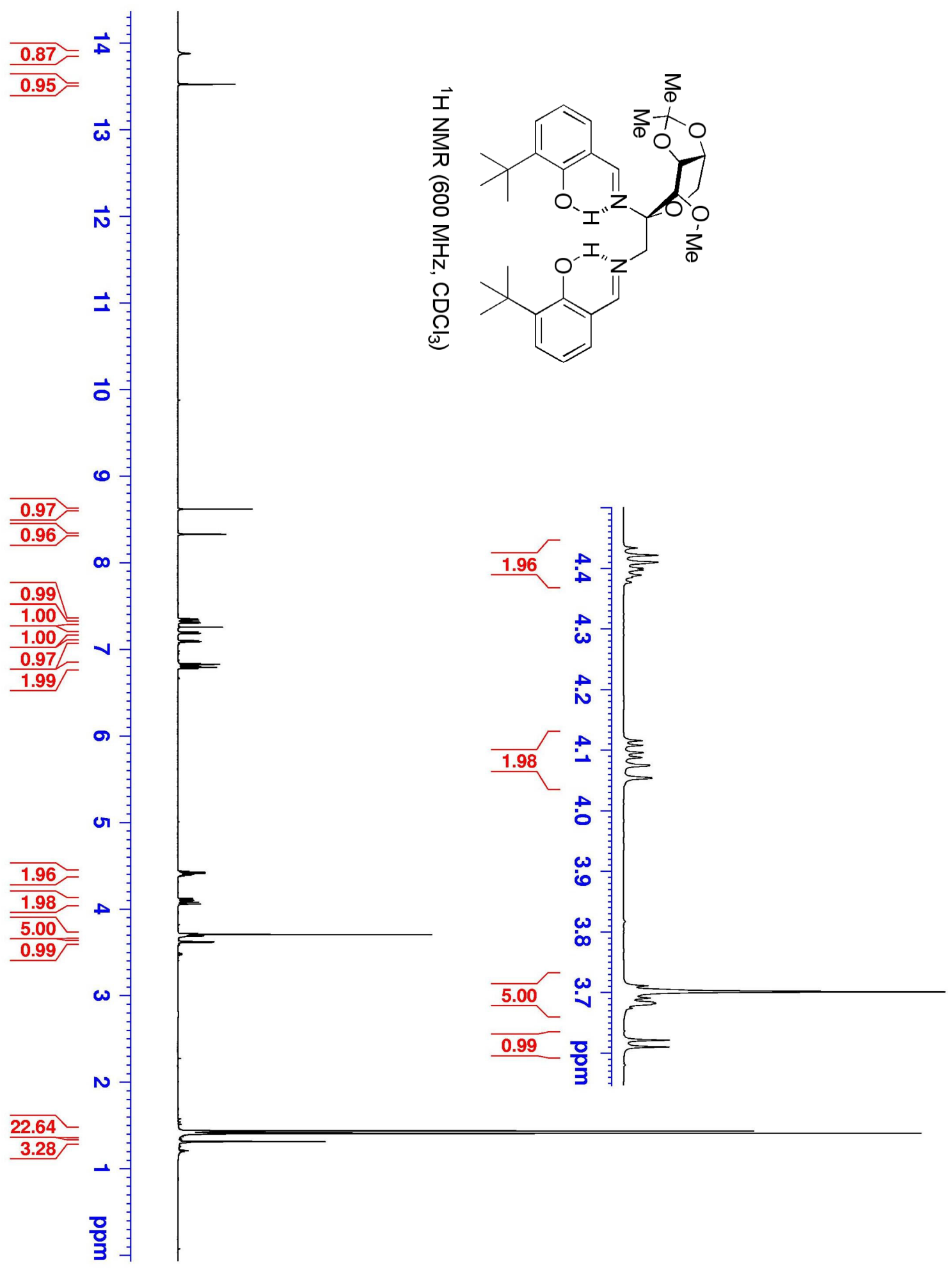




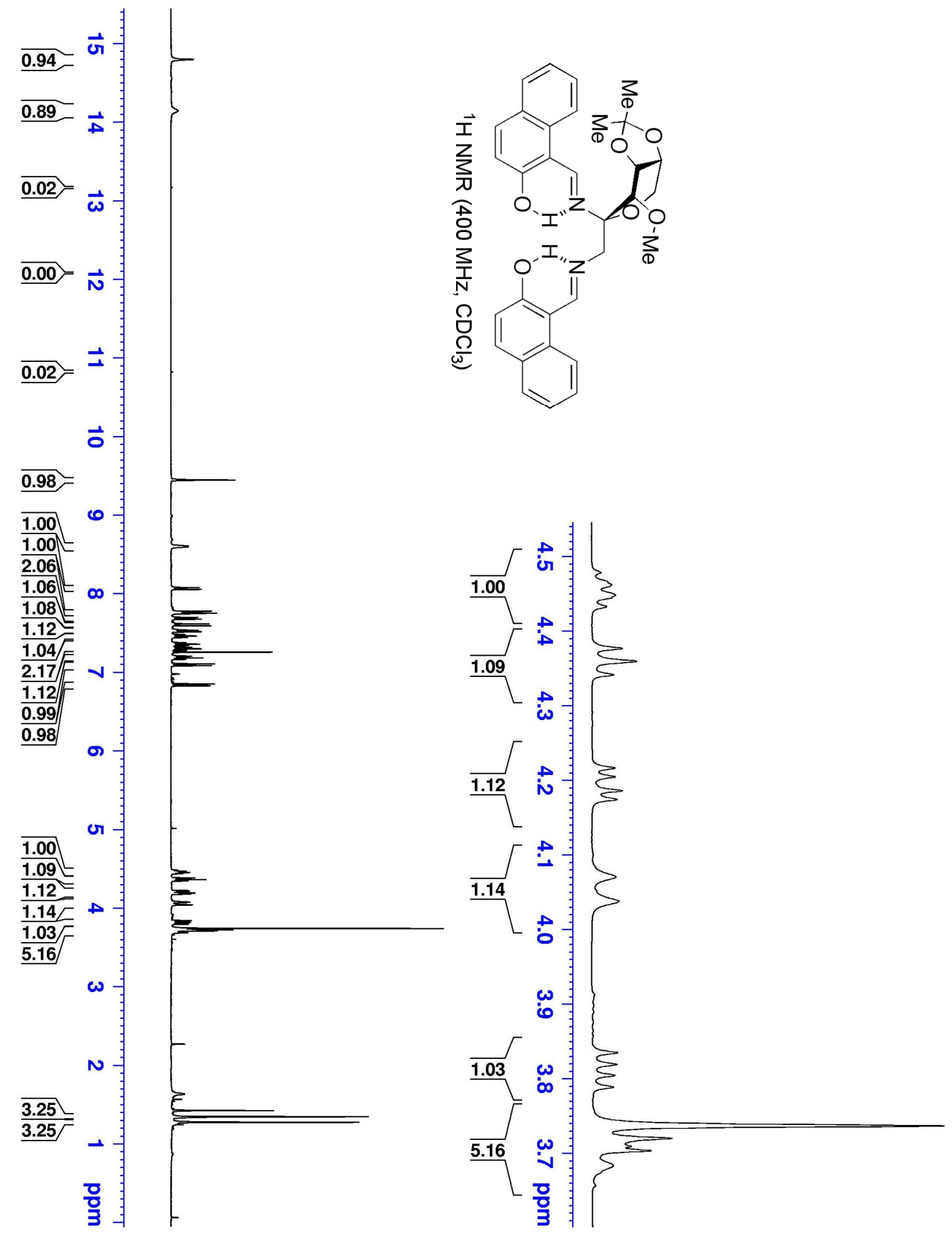


XIII. Representative Chiral HPLC Traces

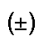

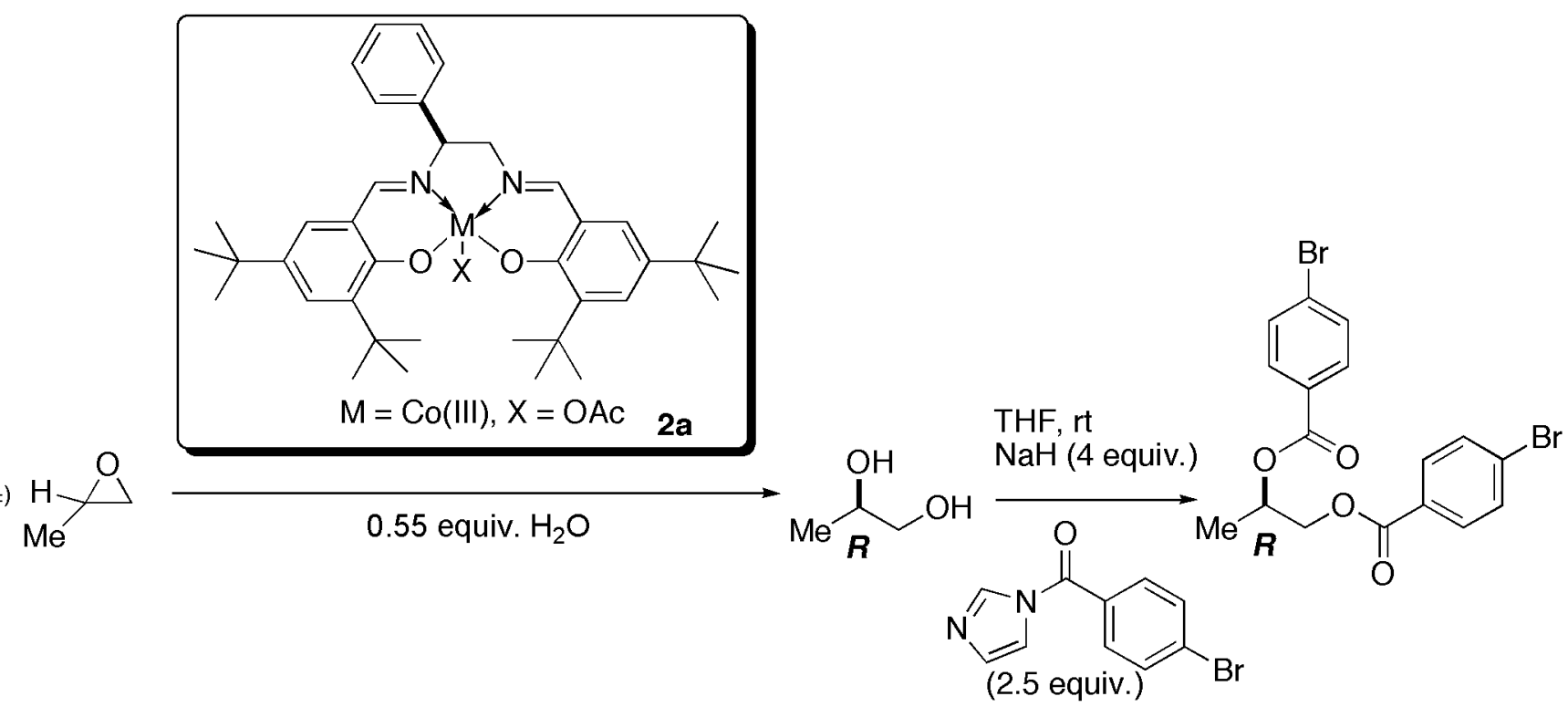

Solvent: $i-\mathrm{PrOH} /$ hexane $(1: 99) ;$ Flow rate $=1 \mathrm{~mL} / \mathrm{min}$

Retention time: $13.9(\mathrm{R})$ and $15.8(\mathrm{~S})$

Integration: $161.7: 5.65$ (93\% ee)

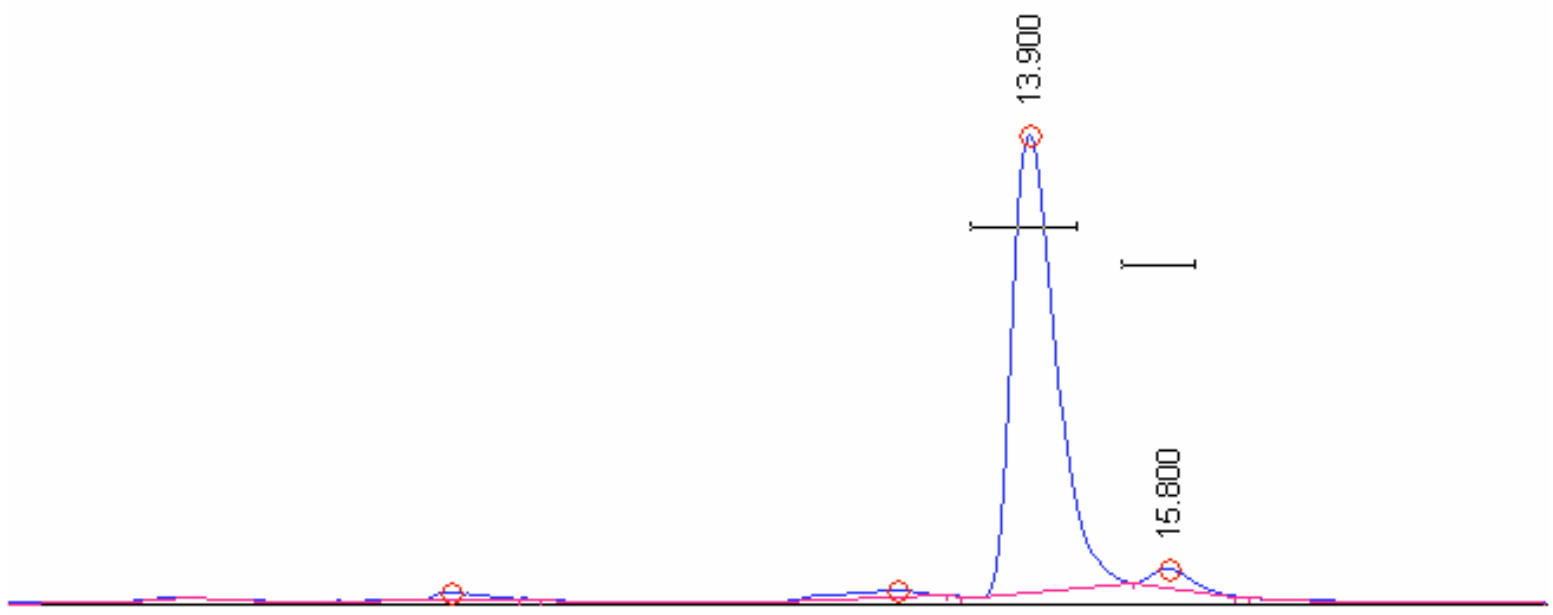




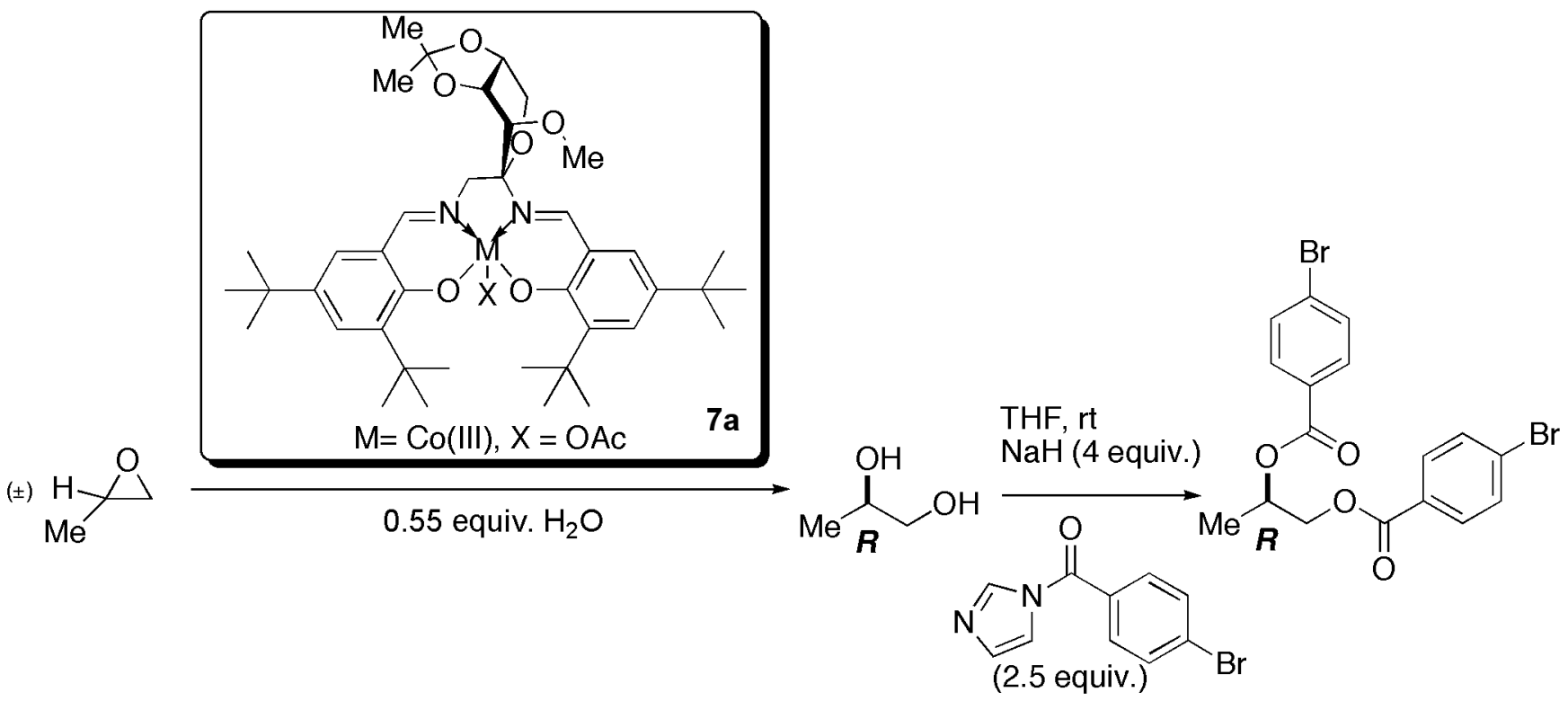

Solvent: $i$-PrOH/hexane (1:99); Flow rate $=1 \mathrm{~mL} / \mathrm{min}$

Retention time: $14.1(\mathrm{R})$ and $16.1(\mathrm{~S})$

Integration: $376.6: 135.6$ (47\% ee)

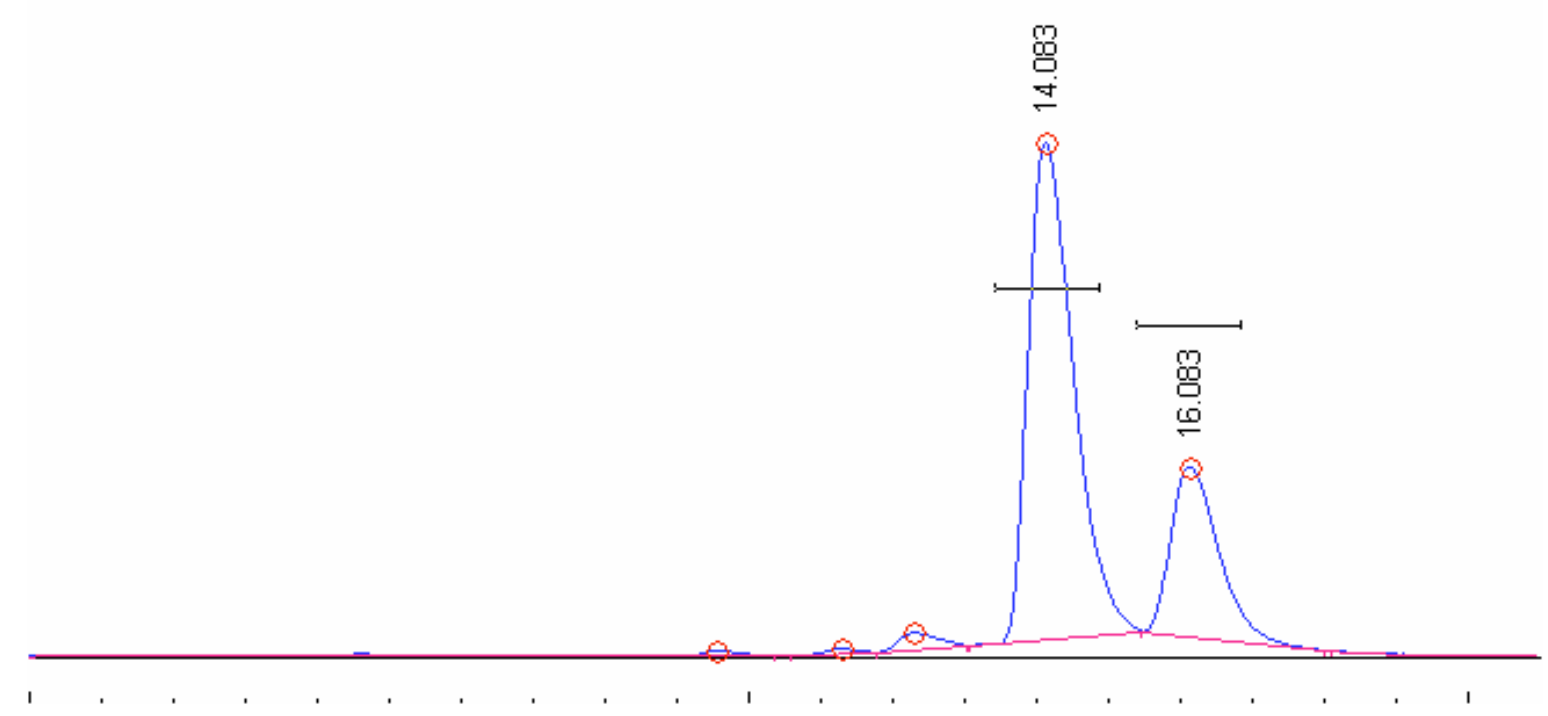




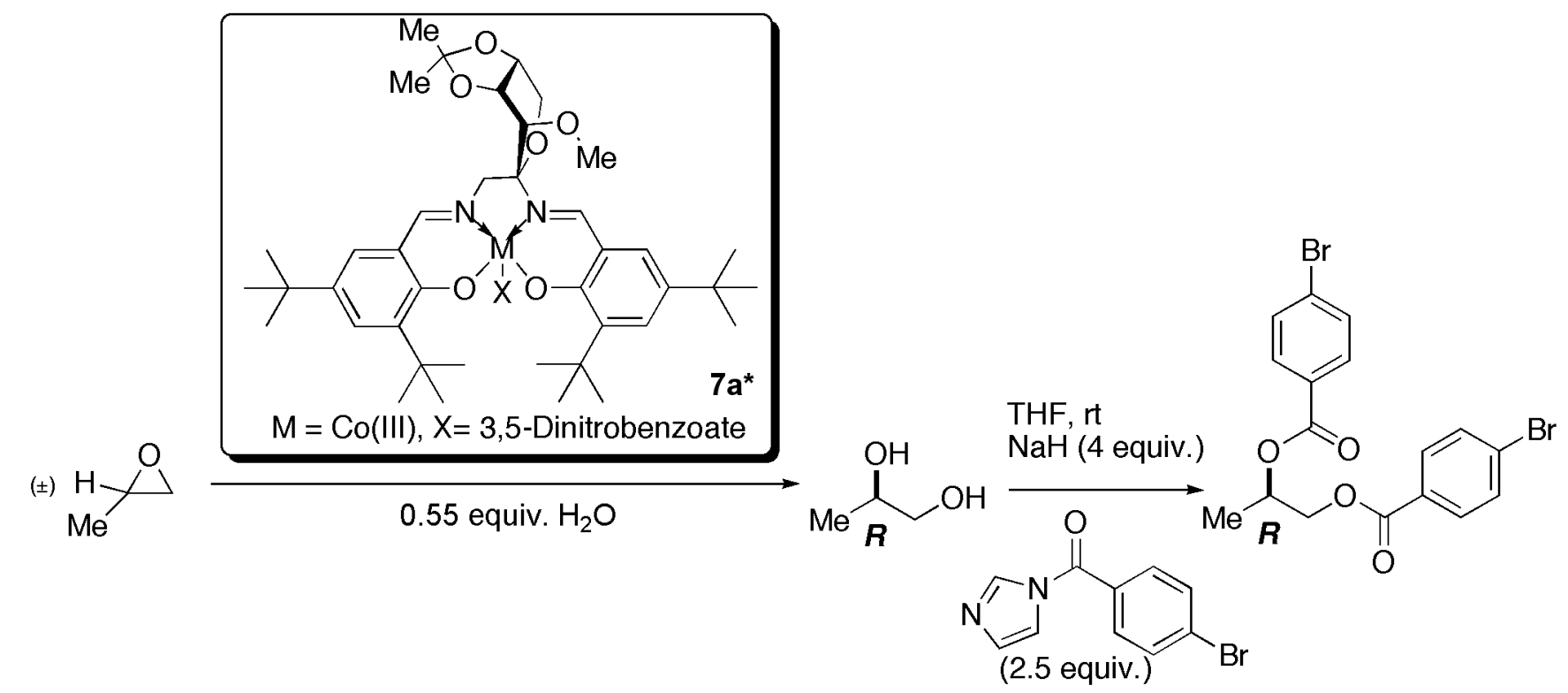

Solvent: $i$-PrOH/hexane (1:99); Flow rate $=1 \mathrm{~mL} / \mathrm{min}$

Retention time: $14.5(\mathrm{R})$ and $16.6(\mathrm{~S})$

Integration: 135.1:29.2 (64\% ee)

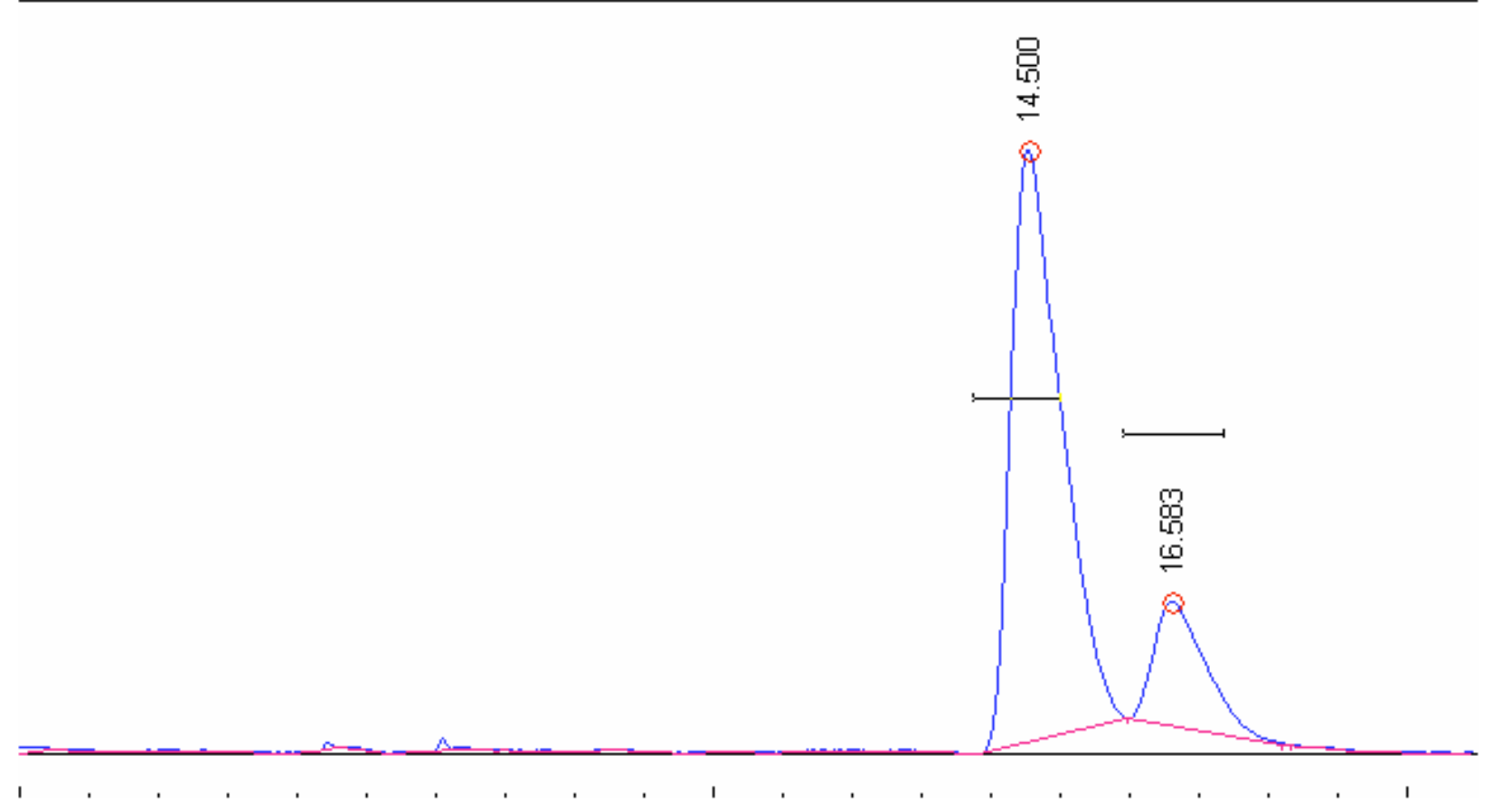


$( \pm)$
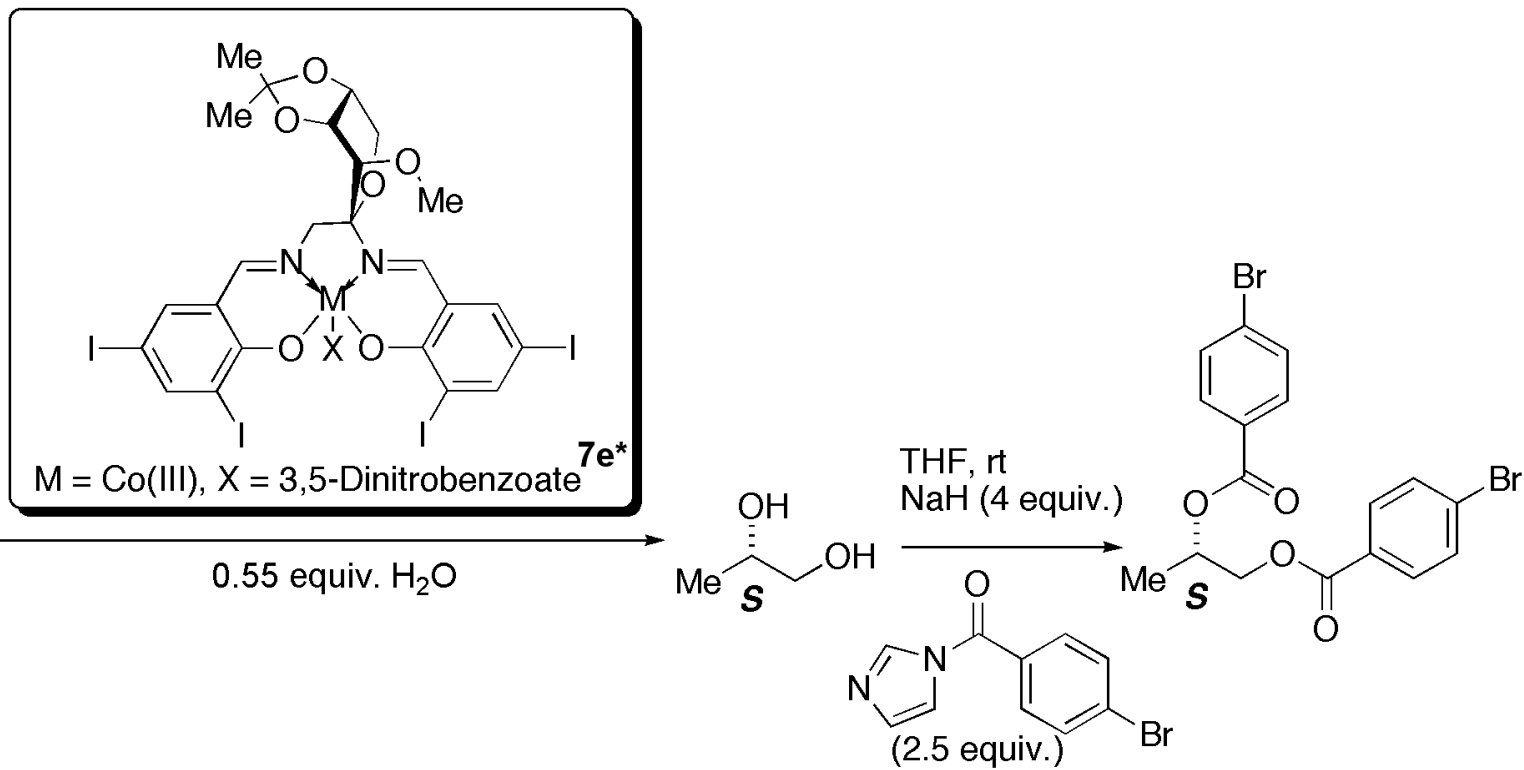

Solvent: $i$-PrOH/hexane (1:99); Flow rate $=1 \mathrm{~mL} / \mathrm{min}$

Retention time: $14.5(\mathrm{R})$ and $16.4(\mathrm{~S})$

Integration: $10.1: 135.3$ (86\% ee)

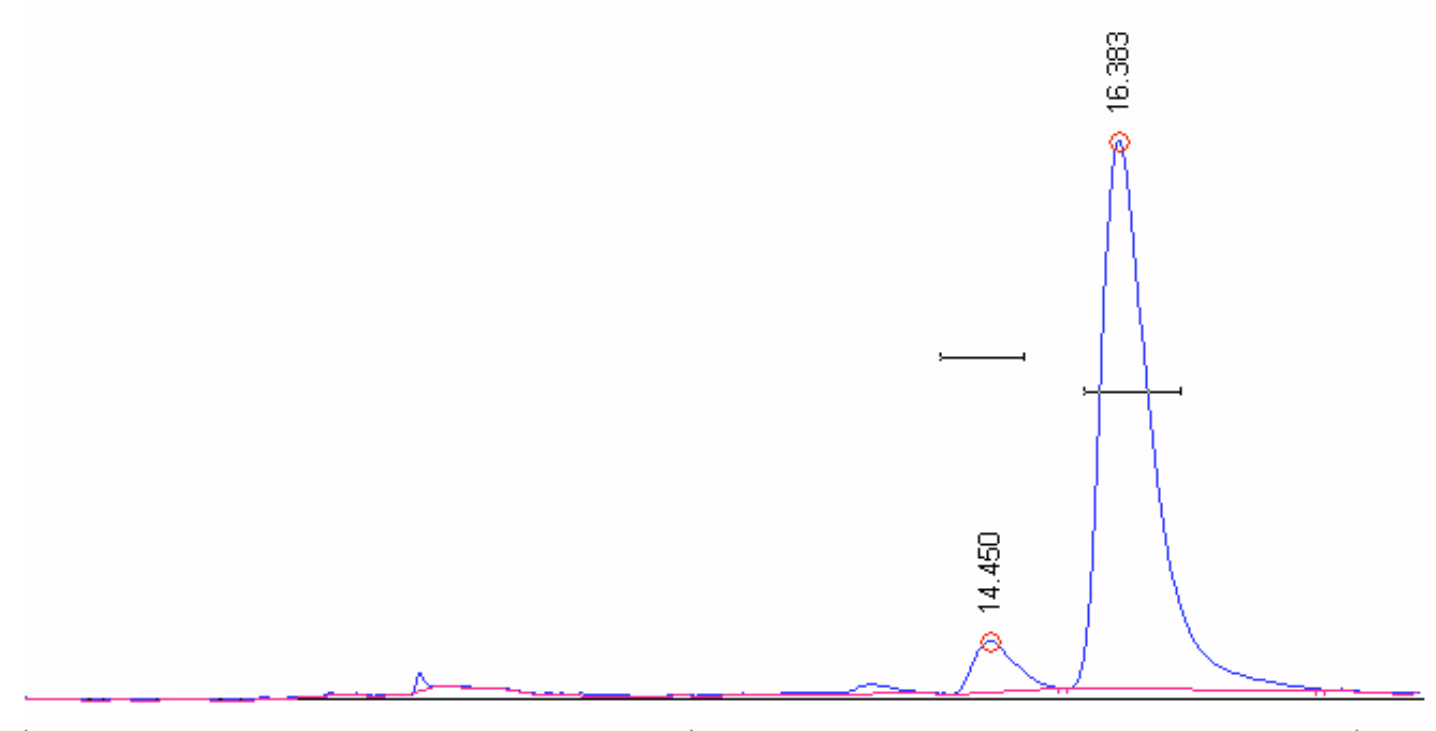


$( \pm)$

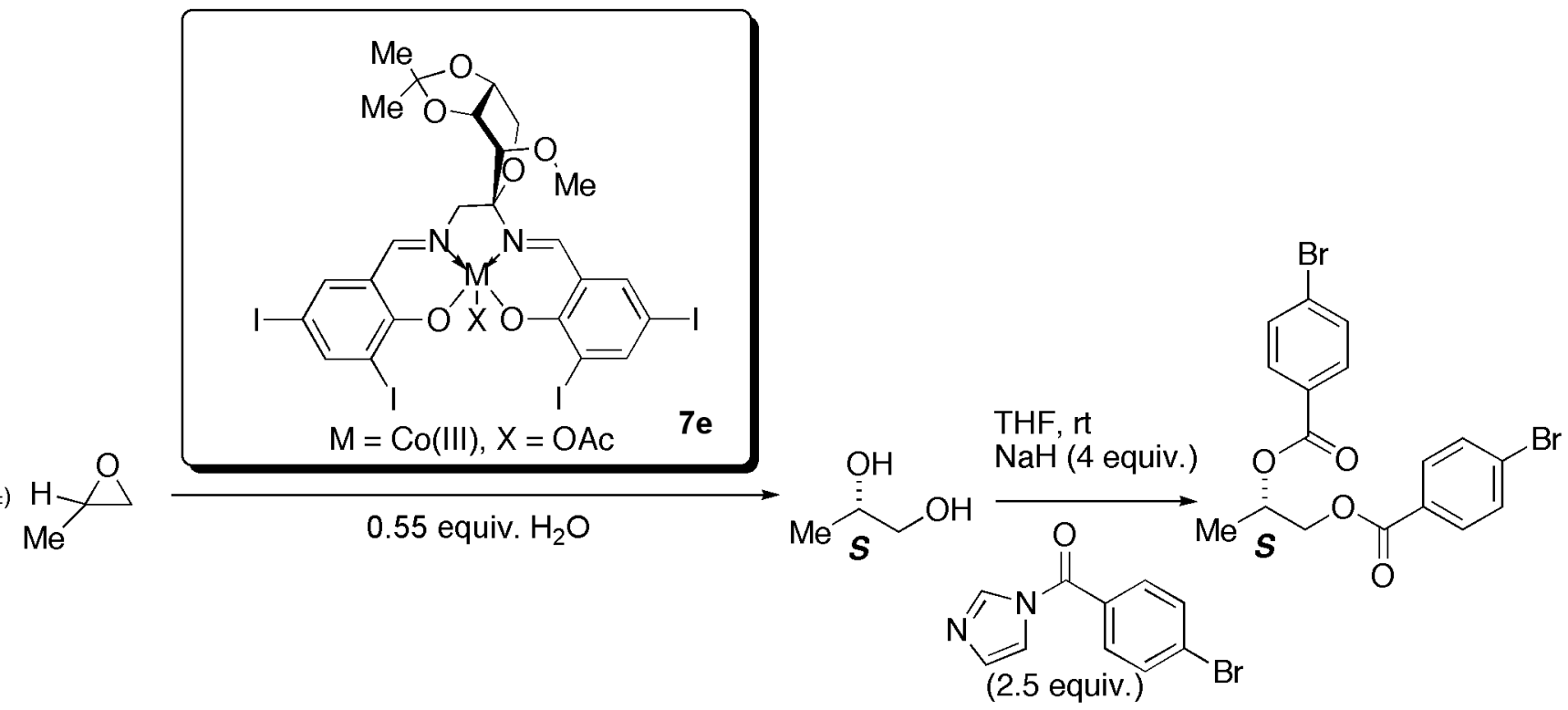

Solvent: $i$-PrOH $/$ hexane $(1: 99) ;$ Flow rate $=1 \mathrm{~mL} / \mathrm{min}$

Retention time: $13.7(\mathrm{R})$ and $15.6(\mathrm{~S})$

Integration: $12.5: 152.2$ ( $85 \%$ ee)

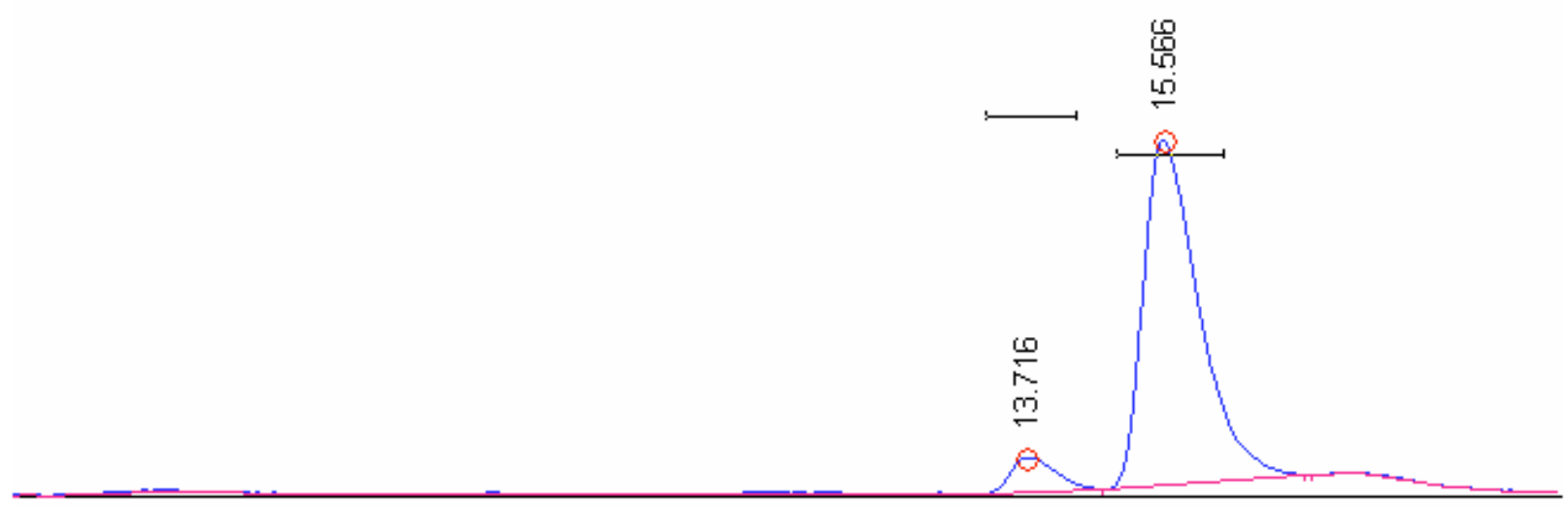

